\title{
Women Offender Assessment: Can Gender-informed Variables Improve Prediction of Institutional Outcomes?
}

\author{
By
}

\author{
Theresia Bedard
}

A thesis submitted to the faculty of Graduate and Postdoctoral Affairs in partial fulfillment of the requirements for the degree of

Master of Arts

In

Psychology

Carleton University

Ottawa, Ontario

(C)2019

Theresia Bedard 


\begin{abstract}
The Custody Rating Scale (CRS) is utilized by Correctional Service of Canada (CSC) in classification of women offenders; it is gender-neutral. The purpose of the study was to examine if gender-informed domains predict misconducts in women offenders. Gender-informed items that were predictive were used to create the Gender-Informed Version 1 Continuous, GenderInformed Version 2 Continuous, Gender-Informed Version 1 Trichotomized and GenderInformed Version 2 Trichotomized scales. The scales were tested to determine if they yield incremental predictive validity beyond the CRS. A sample of 1,528 federally sentenced women was analyzed. Results found addition of gender-informed variables improved the incremental predictive validity of the CRS. This was found for non-Indigenous offenders, but results for Indigenous offenders were mixed. Findings supported the inclusion of gender-informed factors to the CRS. Implications for CSC and future research directions are discussed.
\end{abstract}

Key words: gender-informed predictors, classification, institutional misconduct 
FEMALE OFFENDERS AND INSTITUTIONAL MISCONDUCT

\section{Acknowledgements}

First and foremost, I would like to thank my supervisors Dr. Kelley Blanchette and Dr. Shelley Brown for giving me the opportunity to pursue my Master's, and providing valuable feedback, guidance and support during the process of the thesis work. Thank you both very much for helping me get to this point, and becoming a better researcher in the process. I would also like to thank my committee members and chair as well - Dr. Ralph Serin, Dr. Diana Majury, and Dr. Kevin Nunes, for providing your valuable input into the thesis. I would also like to thank Kaitlyn Wardrop for the all of the support and help that was provided. Thank you very much for helping to answer any questions I had for my thesis, for your patience, and letting me express any worries or concerns I had during the process. I would also like to thank Kayla Wannamaker and Alycia LaPierre for all your help during the process as well. Thank you for taking time out of your schedule to show me how to do some analysis. I would also like to thank Etelle Bourassa

for all your help while I was getting my Masters and providing me with helpful guidance so I can continue moving forward. Finally, I would like to express my gratitude and thanks to my family. Thank you so much for all your support and love. Thank you for the talks, your advice, and just being there when I needed you all. I would like to say a special thank you to my dad, who has helped me through so much in my journey and it means the world to me. 


\section{Table of Contents}

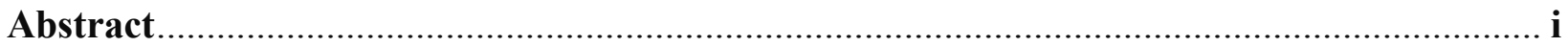

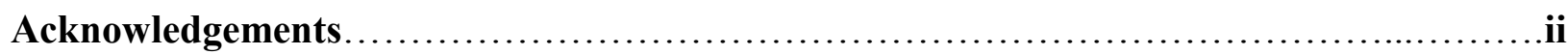

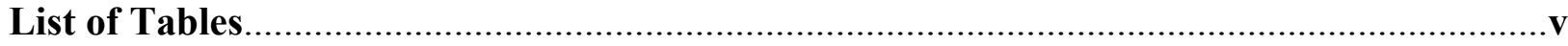

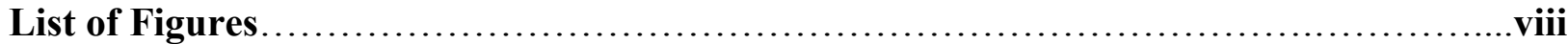

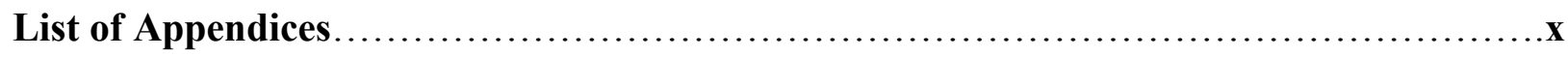

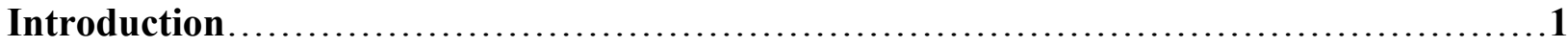

History and Definitions of Classification in General..................................

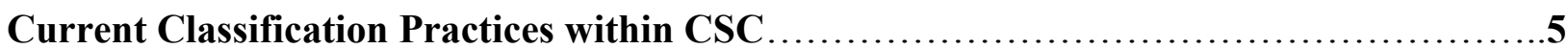

Indigenous Offenders..........................................................

The Custody Rating Scale (CRS; Solicitor General Canada, 1987)........................ 9

Theoretical Perspectives Explaining Misconducts in Prison .........................13

Deprivation and Importation Theory.......................................... 13

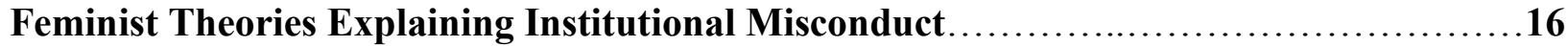

Relational Cultural Theory.................................................... 16

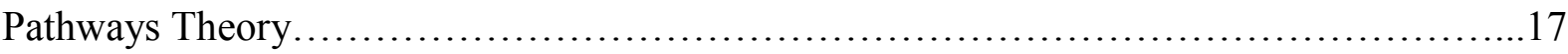

Predictors of Institutional Misconduct in Offenders................................19

Predictors of Institutional Misconduct for Women Offenders............................19

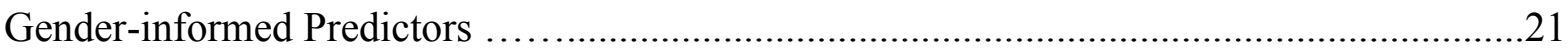

Proposed Gender-informed predictors of institutional misconduct.......................25

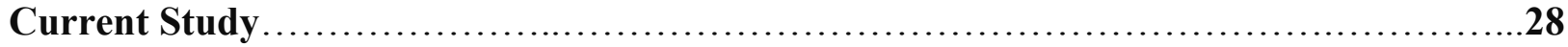

Research Questions and Hypothesis.............................................29

Methods...........................................................................................31

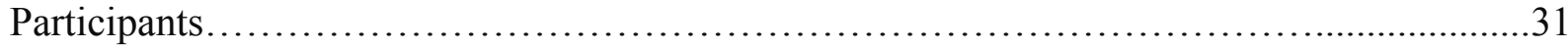

Procedure.............................................................................

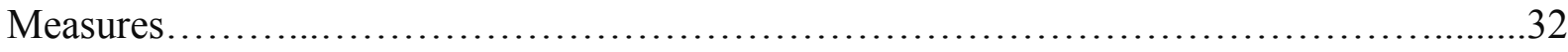

Computerized Mental Health Intake Screening System ...............................33

Offender Intake Assessment.................................................. 34

Dynamic Factor Identification and Analysis-Revised.............................. 36

Women's Computerized Assessment of Substance Abuse ........................... 37

Custody Rating Scale.......................................................... 39 
Gender-informed Variables....................................................... 40

Institutional Misconduct......................................................... 43

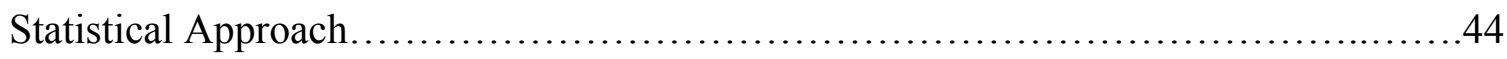

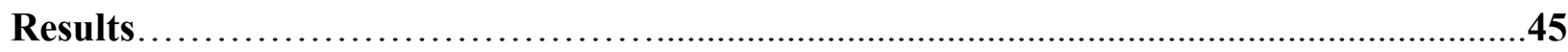

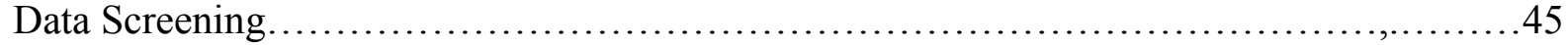

Missing data, outliers, influential cases, and assumptions.............................45

Descriptive Statistics........................................................... 50

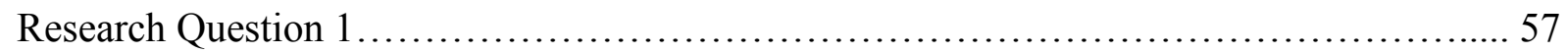

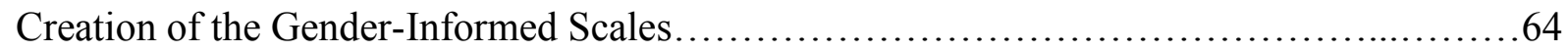

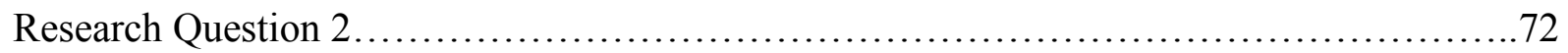

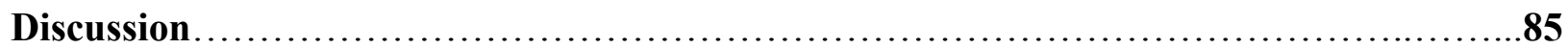

Gender-Informed Predictors of Institutional Misconduct in Women Offenders...............85

Incremental Predictive Validity of Gender-informed Factors to the CRS .....................90

Incremental Predictive Validity of Gender-Informed Factors to the CRS and Indigenous

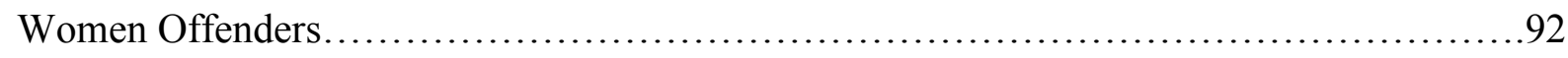

Implications of the Current Study ................................................... 94

Limitations and Future Research..................................................99

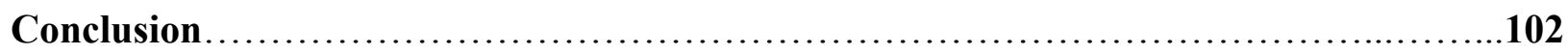

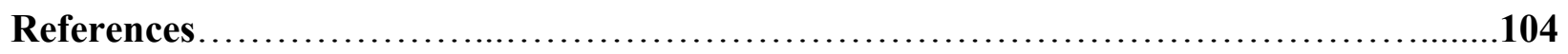


FEMALE OFFENDERS AND INSTITUTIONAL MISCONDUCT

\section{List of Tables}

Table 1. Proposed Gender-informed Predictors

Table 2. Number and Percentage of Women Offenders that Committed Violent or Non-Violent Index Offences for the Total Sample, Indigenous Offenders and non-Indigenous

Offenders

Table 3. Reintegration Level, Accountability Level, and Motivation Level for the Total Sample, Indigenous Offenders, and non-Indigenous Offenders.

Table 4. Final Security Level Designations for the Total Sample, Indigenous Offenders, and nonIndigenous Offenders .53

Table 5. Descriptive Statistics of the Institutional Adjustment Subscale and Security Risk Score for the Total Sample $N=1528$ .54

Table 6. Descriptive Statistics of the Institutional Adjustment Subscale and Security Risk Score by Indigenous Status .54

Table 7. CRS Security Level Designations for the Total Sample, Indigenous Offenders, and nonIndigenous Offenders

Table 8. Mean Number of Misconducts for Total Sample, Indigenous Offenders, and nonIndigenous Offenders

Table 9. Type of Misconducts by Misconduct Type for Total Sample, Indigenous Offenders and non-Indigenous Offenders. .56

Table 10. Survival Analysis of Any Misconduct and the Gender-informed Variable Mental Health with the Total Sample.... .58

Table 11. Survival Analysis of Any Misconduct and the Gender-informed Variable Substance Abuse with the Total Sample .59 
FEMALE OFFENDERS AND INSTITUTIONAL MISCONDUCT

Table 12. Survival Analysis of Any Misconduct with the Gender-informed Variable Relationship Dysfunction with the Total Sample.

Table 13. Survival Analysis of Any Misconduct and Parental/Family Issues with the Total

Sample 62

Table 14. Survival Analysis of Any Misconduct with the Gender-informed Variable

Victimization with the Total Sample.....

Table 15. Survival Analysis of Any Misconduct with the Gender-informed Personal/Emotional

Variable with the Total Sample.

Table 16. Gender-informed Predictors of Institutional Misconducts Used to Create the Genderinformed Scales

Table 17. Bivariate Correlations with Institutional Misconduct for the Total Sample, Indigenous Offenders, and Non-Indigenous Offenders

Table 18. Bivariate Correlations with the Custody Rating Scale for the Total Sample, Indigenous Offenders, and Non-Indigenous Offenders .72

Table 19. ROC analysis of Institutional Misconduct with the Total Sample, Indigenous Offenders and non-Indigenous Offenders .75

Table 20. Formal Comparison of ROC Curves with the Total Sample, Indigenous Offenders, and non-Indigenous Offenders

Table 21. Hierarchal Cox Regression Analysis for the Custody Rating Scale and Continuous

Gender-informed Scales for the Total Sample.

Table 22. Hierarchal Cox Regression Analysis for the Custody Rating Scale and Trichotomized Gender-informed Scales for the Total Sample. 


\section{FEMALE OFFENDERS AND INSTITUTIONAL MISCONDUCT}

Table 23. Hierarchal Cox Regression for the Custody Rating Scale and Trichotomized Genderinformed Scales by Indigenous Status........................................... 83 
FEMALE OFFENDERS AND INSTITUTIONAL MISCONDUCT

\section{List of Figures}

Figure 1. Survival curves for the Gender-Informed Version 1 Trichotomized scale for any misconduct in the total sample.

Figure 2. Survival curves for the Gender-Informed Version 2 Trichotomized scale for any

misconduct in the total sample.

Figure 3. Survival curves for the CRS of any misconduct for the total sample

70

Figure 4. ROC Curves of the Gender-Informed Version 1 Continuous scale and Custody Rating

Scale for any institutional misconduct charges

Figure 5. ROC Curves of the Gender-Informed Version 2 Continuous scale and Custody Rating

Scale for any institutional misconduct charges

Figure 6. ROC Curves of the Gender-Informed Version 1 Trichotomized scale and Custody

Rating Scale for any institutional misconduct charges

Figure 7. ROC Curves of the Gender-Informed Version 2 Trichotomized scale and Custody

Rating Scale for any institutional misconduct charges

Figure 8. ROC Curves of the Gender-Informed Version 1 Continuous scale and Custody Rating Scale for any institutional misconduct charges for Indigenous women

offenders

Figure 9. ROC Curves of the Gender-Informed Version 1 Continuous scale and Custody Rating Scale for any institutional misconduct charges for non-Indigenous women offenders 155

Figure 10. ROC Curves of the Gender-Informed Version 2 Continuous scale and Custody Rating Scale for any institutional misconduct charges for Indigenous women offenders 156 
FEMALE OFFENDERS AND INSTITUTIONAL MISCONDUCT

Figure 11. ROC Curves of the Gender-Informed Version 2 Continuous scale and Custody Rating Scale for any institutional misconduct charges for non-Indigenous women

offenders

Figure 12. ROC Curves of the Gender-Informed Version 1 Trichotomized scale and Custody Rating Scale for any institutional misconduct charges for Indigenous women offenders

Figure 13. ROC Curves of the Gender-Informed Version 1 Trichotomized scale and Custody Rating Scale for any institutional misconduct charges for non-Indigenous women offenders.

Figure 14. ROC Curves of the Gender-Informed Version 2 Trichotomized scale and Custody Rating Scale for any institutional misconduct charges for Indigenous women offenders.

Figure 15. ROC Curves of the Gender-Informed Version 2 Trichotomized scale and Custody Rating Scale for any institutional misconduct charges for non-Indigenous women offenders 


\section{List of Appendices}

Appendix A. Custody Rating Scale..............................................123

Appendix B. Correlation Matrix of Gender-informed Substance Abuse Domain..............127

Appendix C. Pearson Product Moment Correlations of the Gender-informed Substance Abuse

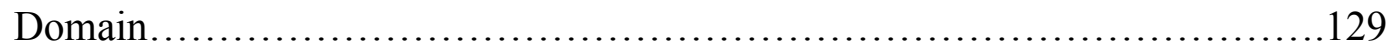

Appendix D. Correlation Matrix of Gender-informed Mental Health Domain................139

Appendix E. Pearson Product Moment Correlations of the Gender-informed Mental Health

Domain............................................................... 140

Appendix F. Correlation Matrix of the Gender-informed Relationship Dysfunction

Domain

Appendix G. Pearson Product Moment Correlations of the Gender-informed Relationship

Dysfunction Domain.............................................. 145

Appendix H. Correlation Matrix of the Gender-informed Parental/Family Issues Domain......147

Appendix I. Pearson Product Moment Correlations for the Gender-informed Parental/Family

Issues Domain.................................................... 148

Appendix J. Correlation Matrix of the Gender-informed Victimization Domain.................150

Appendix K. Pearson Product Moment Correlations for the Gender-informed Victimization

Domain.............................................................. 151

Appendix L. Correlation Matrix for the Gender-informed Personal/Emotional Domain........152

Appendix M. ROC Curves for Any Institutional Misconduct Charges for the Total Sample....153

Appendix N. ROC Curves for Any Institutional Misconduct Charges by Indigenous Status....155 
Women Offender Assessment: Can Gender-informed Variables Improve Prediction of Institutional Outcomes?

Within correctional institutions, female offenders are a minority. Correctional Service of Canada (CSC) — which is responsible for administrating sentences of two years or moreindicates that as of 2015 , female offenders represent only $5 \%$ of federal offenders within Canada (Correctional Service of Canada, 2017). Public Safety Canada (2017) reported a total of 695 women incarcerated in Correctional Service of Canada facilities in 2015-16. Further, over the last ten years, the number of women admitted to federal jurisdiction had increased $21 \%$ from 316 in 2006-07, to 383 in 2015-16 (Public Safety Canada, 2017).

Security classification of offenders is an essential component for correctional institutions (Blanchette, 2002), and has been utilized since the 1800s (Kennedy, 1986). Security classification has evolved from the subjective approach of clinical judgement to actuarial classification models (Austin \& Hardyman, 2004). Research has demonstrated that actuarial methods of classification provide greater predictive accuracy than clinical judgement in various fields of study (Ægisdóttir et al., 2006; Bengtson \& Långström 2007; Harris, 2006). Actuarial methods of classification are superior in predicting criminal behaviour as well (Hanson, 2009; Harris, Rice, Quinsey \& Cormier, 2015). Objective classification instruments are imperative to reduce subjective bias, promote fair treatment of offenders, and provide accurate information for planning accommodation needs of offenders (Luciani, 1997; Luciani, Motiuk \& Nafekh, 1996).

The majority of objective actuarial assessments have been developed with predominately male samples, and were later applied to women offenders (Blanchette \& Taylor, 2007); for example, CSC currently uses the Custody Rating Scale (CRS; Solicitor General Canada, 1987) to assist in determining initial security classification for both men and women offenders. Notably, 
the CRS was originally developed on a sample of predominately men offenders (Rubenfeld, 2014). Brennan and Austin (1997) point out that classification systems developed specifically for women offenders are rare. Classification systems utilized by corrections continue to classify women offenders in accordance with procedures explicitly designed for men offenders and are therefore based on behavioural and risk factors that are predominately relevant to men offenders (Brennan \& Austin, 1997). Blanchette and Taylor (2007) point out that it is even rarer that gender-specific assessments are developed from the ground up for female offenders.

One of the priorities of CSC is to ensure the safety and security of staff and inmates (Correctional Service of Canada, 2016a). Correctional Service of Canada (2008; 2013a) indicates that in regards to offenders, priority is given to the prevention of assaults and violent incidents in institutions. Institutional adjustment is of concern for correctional staff since the way an offender adapts to the correctional setting may have a negative impact on behaviour and may produce an increase in problematic behaviours (Collica, 2010).

Prior research has indicated the percentage of women offenders involved in major institutional misconducts increases from minimum to maximum security (Barnum \& Gobeil, 2012; Blanchette, Verbrugge \& Wichmann, 2002). Further, Blanchette et al. (2002) found in a sample of 334 federally sentenced women offenders, base rates of institutional misconducts revealed that $38 \%$ of the sample were involved in non-violent incidents and $13.1 \%$ were involved in violent incidents. Recent research by Harris, Blanchette and Brown (2014) found in a sample of 951 women offenders, $45 \%$ were involved in minor misconducts, $21 \%$ were involved in serious misconducts, and 50\% were involved in any misconduct.

The majority of research that examines institutional adjustment contains either predominately male offenders or a mixture of both male and female offenders (Reidy, Cihan \& 
Sorensen, 2017). This is problematic, as male-dominated research pertaining to factors that are correlated with, or influence, prison adjustment questions the applicability of findings to female offenders whose socialization and life experiences are different than those of their male counterparts (Reidy et al., 2017). Focus on the institutional experience of female offenders is imperative, as understanding violent or aggressive prison behaviours can allow for better prevention of institutional misconducts, intervention efforts, and post-release success (Blackburn $\&$ Trulson, 2010). Thus, more research is needed to explore institutional misconduct behaviours in women offenders, and focus on identifying potential risk factors for engaging in institutional misconduct is warranted.

Consequently, the goal of this research is to determine if the predictive accuracy of the CRS can be enhanced for federally sentenced women by including variables hypothesized to be more important for women offenders. This paper is organized as follows: First, I describe the history of classification in general and within CSC specifically for men and women. Second, I describe CSC's current initial security classification tool, the Custody Rating Scale (CRS), and review the empirical evidence for men and women offenders. Next, I review three theories to explain inmate behaviour: importation/deprivation theory, relational cultural theory, and pathways theory. I will critically evaluate the corresponding evidence for each theory with a focus on women. Next, I will describe empirical data regarding predictors of misconduct. Finally, I will summarize the key findings and present study objectives and methods.

\section{History and Definitions of Classification in General}

Since classification has been used as early as the 1800 s, these classification typologies were influenced by Darwin's evolutionary theory and proposed by Lombroso. Offenders were classified into five types: born criminal, criminal by passion, insane criminal, habitual criminal, 
and occasional criminal. Further, women offenders were classified based on assessing their physical features, and were distinguished as women of good life, the fallen class, lunatics, and female criminals (Lombroso \& Ferrero, 1895).

Farr (2000) indicates the main purpose of classification is to determine the level of risk an inmate poses to the correctional institution and the community. The author suggests that classification must provide the least restrictive security designation while allowing for custodial order to be upheld and the prevention of escape and risk to the community. It should be noted that security classification is a specialized risk assessment that entails specific criteria (Blanchette, 2002). That is, risk assessments are utilized to predict the likelihood of recidivism (risk) in the community, while security classification is utilized to assess risk within the correctional institution (Blanchette, 2002).

The gender-neutral approach has been dominant in the classification of female offenders, and has laid the foundation for the majority of current measures (Salisbury, Van Voorhis, \& Spiropoulos, 2009). Gender-neutral assessments are intended to be used for male and females (Andrews, Guzzo, Raynor, Rowe, Rettinger, Brews \& Wormith, 2012); however, some argue these assessments were created and tested primarily for males and applied to female offenders later (Bloom, Owen \& Covington, 2003; Van Voorhis, Wright, Salisbury \& Bauman, 2010). The gender-neutral approach, which considers that risk factors that predict crime are equally applicable for both men and women (Brown, 2017), has been criticized for studies that contain female offenders as a subgroup and failure to disaggregate the data by gender (Blanchette \& Brown, 2006).

Conversely, the gender-informed approach (Blanchette \& Brown, 2006), or genderresponsive (Bloom et al., 2003), which is based on feminist theoretical perspectives (Hubbard \& 
Matthews, 2008), emphasizes that women's pathways into crime are different than those for men (Rettinger \& Andrews, 2010). Gender-informed scholars suggest that female offenders are different from males; pathways to crime in female offenders are different as evidenced by the fact that females engage in less serious forms of crime, and females have more prevalent needs such as substance abuse, victimization, mental health, and children/marital status (Bloom et al., 2003). Gender-informed scholars suggest that female-specific needs include:

victimization/abuse, relationship dysfunction, mental health issues, substance abuse, poverty/economic marginalization, parental/family issues, and self-efficacy (Blanchette, 2004; Chesney-Lind, Morash \& Stevens, 2008; Salisbury et al., 2009; Van Voorhis, et al. 2010). For the purpose of this paper, gender-informed means informed by theory/empirical research relevant to women, while the gender-neutral approach refers to a male-based perspective in the assessment of offender populations (Gobeil \& Blanchette, 2007). It should be noted that gendersalient predictors are applicable to both men and women but the magnitude of the relationship is stronger for one gender in comparison to the other; while gender-specific predictors predict crime for one gender but not the other (Brown, 2017).

\section{Current Classification Practices within CSC}

When offenders initially arrive in CSC custody, a comprehensive assessment process is completed (Correctional Service of Canada, 2016b). This process is the Offender Intake Assessment (OIA), which was implemented in 1994 for all regions of CSC (Motiuk, 1997). Part of this process entails initial security classification. Initial security classification occurs when offenders enter an institution and includes using the CRS to inform initial security classification decisions (Correctional Service of Canada, 2018a). As per the Corrections and Conditional Release Act (CCRA, 1992), the classification designation must reflect the offender's institutional 
adjustment, escape risk, and the risk to the public if an escape occurs. After the initial security classification, the CCRA (1992) outlines the offender's security level must be reviewed annually. Further, the domains of institutional adjustment, escape risk, and risk to the public if escape occurs are each given a rating of either low, moderate or high to assess the inmate security level (Correctional Service of Canada, 2018c). Reclassification is necessary to facilitate integration to lower levels of security which generally leads to reintegration into the community (Gobeil, 2008). The process of reclassification utilizes different tools based on the inmate's gender - the Security Reclassification Scale (SRS; Luciani, Taylor \& Motiuk, 1998) for men offenders and the Security Reclassification Scale for Women (SRSW; Blanchette, 2005) for women offenders.

It should be noted that the SRSW is currently used by Correctional Service of Canada as a gender-informed reclassification instrument for federally sentenced women offenders (Gobeil \& Blanchette, 2007). It was nationally implemented in September 2005 and consists of nine dynamic factors: progress or motivation of correctional plan, prosocial/positive family contact, serious disciplinary offences, number of recorded incidents, current institutional pay level, involuntary segregation, number of successful temporary absences, prior history of being unlawfully at large, and Custody Rating Scale incident history (Gobeil \& Blanchette, 2007). The SRSW has been found to be a reliable and valid gender-informed scale for the reclassification of federally sentenced women offenders (Blanchette \& Taylor, 2007; Gobeil \& Blanchette, 2007).

Until the mid-1990s, only one federal prison for female offenders in Canada existed - the Prison for Women (P4W), which opened in 1934 in Kingston, Ontario (Blanchette, 1997; 2002). Despite that the P4W was designated as multi-level security, it was built to maximum security standards, and regardless of the security classification, every federally sentenced woman served her sentence at the P4W (Correctional Service of Canada, 2013b). Criticisms of the P4W were 
repeatedly put forth, but a major criticism was in regards to the sole maximum security infrastructure; although roughly fewer than $10 \%$ of female offenders were classified as maximum security inmates (Blanchette, 2002).

In response to the recommendations of the Task Force on Federally Sentenced Women (1990), Correctional Service of Canada now operates six women's institutions across Canada that are all multi-level security (Correctional Service of Canada, 2013c; 2017). Multi-level institutions house women that are classified as minimum, medium and maximum security (Correctional Service of Canada, 2013c; 2017). Currently, minimum and medium security environments for women offenders provide a fairly different living accommodation than that for women in maximum security environments. For instance, women offenders classified as minimum or medium security live in housing units with communal living spaces. Minimum/ medium security provides an environment that supports personal development, responsible behaviour, and interaction with others. The communal living environment also provides an environment that encourages offenders to be more responsible for their daily life and allows for more interaction among inmates. In contrast, female offenders designated as maximum security are accommodated in secure units, where movement is monitored and a high level of monitoring and intervention is provided by correctional staff (Correctional Service of Canada, 2013c).

\section{Indigenous Offenders}

As of 2016, Indigenous people of Canada comprise $4.9 \%$ of the total population of Canada. Despite the low proportion of Indigenous people in the Canadian population, Indigenous people are over-represented within all levels of the Canadian correctional system (CSC, 2017). In regards to the Indigenous women offender population, Public Safety Canada (2017) specifies 
that Indigenous women offenders represent $36.6 \%$ of all women offenders in custody, while their Indigenous men offender counterparts represent $26.3 \%$ of all men offenders in custody.

Due to this over-representation, considerable debate exists with the applicability of risk assessments to Indigenous offenders (Gutierrez, Helmus \& Hanson, 2017). As such, concern exists for the classification of women offender populations that are part of a minority, such as Indigenous women offenders (Barnum \& Gobeil, 2012). The overall approach to the classification of Indigenous women offenders is to reflect their social history (Barnum \& Gobeil, 2012). Staff must address the Gladue decision (R. v. Gladue, 1999) in accordance to policy and consider as part of their professional judgement the unique systemic or background factors that affect the lives of Indigenous people (CSC, 2018a). The factors voiced by Indigenous youth that contribute to the social history of Indigenous people include the impact of colonialism and residential schools, a loss of tradition/culture, racism/stereotypes, family disruption/breakdown due to forced removal of Indigenous children, and a loss of identity/sense of self (Cesaroni, Grol \& Fredericks, 2018).

Prior research has examined the characteristics of Indigenous and non-Indigenous women offenders. The literature indicates that Indigenous women offenders tend to be younger, less educated, and similarly married in comparison to their non-Indigenous women offender counterparts (Beaudette, Cheverie \& Gobeil, 2014). Further, Indigenous women offenders were more likely to be convicted of a violent offence and serve an indeterminate sentence (Beaudette et al., 2014; Thompson \& Gobeil, 2015). Beaudette et al. (2014) found that Indigenous women offenders were also more likely to present high levels of static and dynamic risk; as well as demonstrating more criminogenic needs in the domains of personal/emotional, associates, employment, substance abuse and marital/family than their non-Indigenous women offender 
counterparts. Furthermore, in Thompson and Gobeil's (2015) study of 626 women offenders admitted to $\operatorname{CSC}(N=174$ were Indigenous offenders $)$, over half of the Indigenous women offenders in this sample reported having a family member that attended residential school. Approximately half of the Indigenous women offenders in this sample also reported being removed from their family home (e.g., through adoption or placement in foster home), while almost all of the Indigenous women offenders had a history of traumatic experiences.

Considering that Indigenous offenders exhibit concerning social histories and are a unique minority within correctional systems, it is imperative to be cognizant of Indigenous offenders in correctional research and security placements.

The Custody Rating Scale (CRS; Solicitor General Canada, 1987). In 1988 Correctional Service of Canada introduced the Custody Rating Scale (CRS) to provide a standardized, objective classification tool to inform initial security placement of federal offenders (Blanchette et al., 2002). The CRS is composed of two independently scored subscales: the Institutional Adjustment scale which consists of five items, and Security Risk scale which consists of seven items. The recommended security classification is based on the total score of the sub-scales, and as scores increase for each sub-scale, higher security classifications are suggested.

Researchers have found the CRS demonstrates good support for reliability of the Institutional Adjustment scale and fair support for the Security Risk scale (Luciani, Motiuk \& Nafekh, 1996); as well as good predictive validity (Grant \& Luciani, 1998; Luciani et al., 1996; Luciani, 1997). Further, Blanchette et al. (2002) investigated the predictive validity of the CRS in a sample of 334 federally sentenced women offenders. The authors found that the Institutional Adjustment scale of the CRS had moderate to strong correlations with both violent and general 
institutional misconducts in Indigenous and non-Indigenous female offenders. The Security Risk scale of the CRS also demonstrated a significant relationship to general and violent misconducts in non-Indigenous female offenders. Analysis suggested that the CRS designations were significantly associated with general misconducts $\left(\chi^{2}(2, \mathrm{~N}=291)=11.38, p<.01, \varphi_{c}=.19\right)$. Recent research has also demonstrated support for the CRS in women offenders. For instance, Barnum and Gobeil (2012) investigated a revalidation of the CRS in a sample 628 women offenders. The sample was divided by Indigenous (women offenders self-identified as First Nations, Inuit or Metis) and non-Indigenous offenders. ROC analysis provided some support for the predictive validity of the CRS with respect to institutional misconduct in a 3month follow-up. AUCs of .50 are considered no better than chance; whereas AUCs of .60 or greater are considered 'acceptable' and AUCS of .70 or greater are considered 'good' (Rice \& Harris, 2005; Rice \& Harris, 1995). For Indigenous offenders, the CRS demonstrated the ability to predict major institutional misconducts $(\mathrm{AUC}=.71)$, but not minor misconducts (AUC $=.51)$. For non-Indigenous offenders, the CRS demonstrated some ability to predict minor institutional misconduct $(\mathrm{AUC}=.58)$, but not major institutional misconducts $(\mathrm{AUC}=.54)$. Overall, the findings demonstrate the CRS had little predictive validity in minor misconducts, but demonstrated good predictive validity for major misconducts in Indigenous offenders.

Rubenfeld (2014) conducted a study with a sample of 1,083 women offenders (286 were Indigenous and 797 were non-Indigenous) to explore the reweighting of the CRS to determine if improvements can be made to the validity of the CRS. Reweighting of the CRS was achieved using the Burgress method (Nuffield, 1982), which examines the extent to which individual response options are more or less associated with the intended predictive outcome of a scale. For women offenders, Rubenfeld (2014) determined 'institutional adjustment' was the only viable 
outcome to include in the reweighting of the CRS; 'escape risk' and 'risk to the public in the event of an escape' were not viable outcomes due to the infrequent amount of escapes of women offenders. Rubenfeld (2014) found a significant association between institutional misconduct and the original (unweighted) CRS $\left(\chi^{2}(2)=8.80, p<.05, \varphi_{\mathrm{c}}=.13\right)$. However, an increase in the magnitude of the significant associations was demonstrated using the reweighted CRS $\left(\chi^{2}(2)=\right.$ $\left.19.07, p<.0001, \varphi_{\mathrm{c}}=.19\right)$. Further, ROC analysis that examined institutional misconducts in a three month period revealed the reweighted CRS improved the prediction of misconducts (AUC $=.61 ; 95 \% \mathrm{CI}[.54, .68])$ in comparison to the original $\mathrm{CRS}(\mathrm{AUC}=.54 ; 95 \% \mathrm{CI}[.46, .61])$.

To determine if applying the reweighted CRS to a different subsample of women offenders would produce similar improvements, the predictive accuracy was examined in a validation sample. A significant association was found between institutional misconduct and the original $\operatorname{CRS}\left(\chi^{2}(2)=18.76, p<.0001, \varphi_{\mathrm{c}}=.19\right)$, while a slight increase in the maginitude of the significant association was found in the reweighted CRS $\left(\chi^{2}(2)=26.20, p<.0001, \varphi_{\mathrm{c}}=.22\right)$. ROC analysis demonstrated slight improvement of predicting misconducts in the reweighted $\mathrm{CRS}(\mathrm{AUC}=.62 ; 95 \% \mathrm{CI}[.55, .69])$ in comparison to the original $\mathrm{CRS}(\mathrm{AUC}=.60 ; 95 \% \mathrm{CI}$ $[.53, .67])$. Thus, Rubenfeld (2014) found support for the reweighting of the CRS as it demonstrated improvement in the predictive validity of institutional misconducts.

These studies provide moderate support for the validity of the CRS in women offenders. However, there are limitations to these studies. For instance, the study conducted by Blanchette et al. (2002) examined predictive validity using point-biserial correlations. This can be problematic, as more rigorous statistical analysis to determine predictive validity is warranted. Further, Luciani et al. (1996) also examined a total sample size of 14,114 offenders, which 
consisted of only 65 female offenders. This small sample size is a limitation to the applicability of the CRS to female offenders, and can limit the generalizability of the findings.

The more recent research conducted by Barnum and Gobeil (2012) and Rubenfeld (2014) also reveals limitations. Despite that the majority of AUC values deemed 'acceptable' ranged between .60 to .66 , these values are not considered in the range of 'good' (.70 or greater). In fact, considering the value of .50 indicates discrimination no better than chance, the AUC values indicate the CRS and reweighted CRS predict institutional misconducts only marginally better than chance. Rubenfeld (2014) notes these findings indicate a limitation of the predictive validity in the CRS and reweighted CRS in women offenders. In fact, Rubenfeld (2014) suggest an alternative approach to the reweighting of the CRS would be to develop a gender-informed scale specifically for women offenders, and future research in regards to a gender-informed instrument could explore items that would best predict institutional misconducts for women offenders.

As noted, the original CRS development sample was composed of predominately men offenders (Auditor General Canada, 2017; Luciani et al., 1996), and was subsequently used for women. This is problematic as the applicability of the CRS is based on the assumption that risk factors for men and women are comparable (McKeown, 2010). Interestingly, CSC does use a gender-informed tool - the Security Reclassification Scale for Women -- for security reclassification (Blanchette, 2005). However, the Auditor General of Canada (2017) points out CSC has not implemented an initial classification tool developed specifically for women and continues to use the CRS.

The majority of assessment measures in forensic psychology has been developed primarily with male samples (e.g., Psychopathy Checklist-Revised, Hare, 2003; Historical-Clinical-Risk Management-20, Webster, Douglas, Eaves \& Hart, 1997; Level of Service Inventory-Revised, 
Andrews \& Bonta, 1995), and debate exists in regards to the contention that women offenders differ from men offenders. Gender-informed perspectives (Blanchette \& Brown, 2006) contend that women offenders are different from men offenders. Gender-informed perspectives consider needs pertaining to the continuation of crime are more impactful on women's behaviour than men (Gobeil, Blanchette \& Stewart, 2016).

Considering that the CRS has been developed with male offenders, further research is warranted to investigate if the addition of gender-informed variables might improve the genderneutral CRS. Since a fundamental objective of security classification entails the minimization of institutional misconducts, an important consideration to assessing the validity of a classification instrument is the prediction of institutional misconducts and violent misconducts (Blanchette, 2002). To date, research has not explored whether the addition of gender-informed variables to the CRS will enhance the predictive accuracy of institutional misconducts in women offenders. This study will address this gap in the literature by exploring potential gender-informed variables that may enhance the predictive accuracy of the CRS.

\section{Theoretical Perspectives Explaining Misconducts in Prison}

Engaging in inmate misconduct reflects offending behaviours within the institutional setting (Steiner, Butler \& Ellison, 2014). Consequently, researchers have utilized general theories of crime and deviance to assist in explaining why offenders engage in institutional misconducts. As such, major theoretical perspectives of crime will be discussed below and connection to female offenders' institutional adjustment will be outlined. These perspectives can also elucidate any potential predictors of institutional misconduct for female offenders.

\section{Deprivation and Importation Theory}


Deprivation theory suggests that the prison environment itself leads to a trajectory of misbehaviour in inmates (Sykes, 1958). Sykes (1958) proposes that experiences of loss, deprivation, and discomfort in prison creates difficulties adjusting to the prison setting. This is applicable to every offender, regardless of individual characteristics.

Conversely, proponents of importation theory suggest the personal attributes offenders embody, such as attitudes or personality traits, influence one's behaviour within institutional settings (Irwin \& Cressey, 1962). These theories have been tested empirically within the correctional literature; however, the majority of these studies have been conducted with male offender samples (Lahm, 2016; 2017).

To date, only one study conducted by Gover, Pérez and Jennings (2008) has specifically tested the extent these theories may or may not apply to males and females. The sample consisted of 247 inmates (190 males and 57 females). To measure importation theory, nine variables (age, race, education, children, prior incarceration, nonviolent offence, violent offence, age at first arrest and low self-control) were used. To measure deportation theory, eight variables (medium security, maximum security, sentence length, length of stay, prison job, quality of life, staff or inmate care, and safety) were used. They found measures from importation and deprivation theories predict institutional misconducts in male and female offenders; however, predictors of institutional misconducts were different for male and females.

Multivariate analysis revealed four importation measures were significantly associated with institutional misconduct in females: older age and prior incarceration were related to fewer misconducts; while minority status and lower education were related to an increase in misconducts. For males, four importation measures were significantly associated with institutional misconduct: prior incarceration and low self-control were related to greater 
misconducts; while in comparison to being incarcerated for a drug offence, offenders that were incarcerated for a nonviolent and violent current offence demonstrated a $56 \%$ and $46 \%$ decrease, respectively, in institutional misconducts.

Four deprivation measures were significantly associated with institutional misconduct in females: longer sentence length, shorter length of stay and perception of safety were related to a decrease in misconducts and positive perception of staff was related to an increase in misconducts. Four deprivation measures were significantly associated with institutional misconduct in males: medium security, maximum security, and greater length of stay were related to an increase in misconducts; while having a prison job was related to a decrease in misconducts. The authors note it is intriguing that positive perceptions of staff predicted misconducts for females only. They suggest females are more likely to develop friendships with correctional staff, which may undermine the authority of correctional staff, leading to inmates being more likely to commit misconducts.

The differential effects of the importation and deprivation models for males and females revealed that history of incarceration and shorter length of stay were the only two predictors that were significant for males and females. Prior incarceration was found to increase the likelihood of institutional misconducts in males, but decrease the likelihood of institutional misconducts in females. Gover et al. (2008) suggest females with prior experience of incarceration were better able to adjust to institutional life as they may be more receptive to consequences administered by correctional staff. They suggest it is possible length of stay had a stronger effect on females due to the different types of stressors experienced while incarcerated. Gover et al. (2008) note victimization experiences and mental health concerns are possible contributors to stress for 
female offenders. Deprivation and importation theories are likely applicable to female offenders, but gender may influence the saliency of potential predictors of institutional misconduct.

Feminist Theories Explaining Institutional Misconduct

Relational-Cultural Theory (RCT). Deriving from Jean Baker Miller's book entitled, Toward a New Psychology of Women, Relational-Cultural Theory (RCT) posits that women gravitate towards and experience growth through connections with others. Specifically, Jean Baker Miller outlines five key components to relationships that promote growth: (1) an increased ability to take action (empowerment), (2) an increase in vitality (zest), (3) an increase in selfworth, (4) an increase in clarity regarding oneself, the other, and relationships, and (5) desiring relationships beyond the current relationship with the other. However, within relationship dynamics disconnection is likely to occur; this can be due to violations in relationships, failure to utilize empathy, injuries, or any other matter that does not foster growth/connection.

Research provides evidence that relationships for females contribute to the onset of criminal behaviours and the continuation of crime (e.g., Bui \& Morash, 2010). Research supports that intimate relationships are more influential for females than males in recidivism (Cobbina, Huebner \& Berg, 2012), and partner antisociality increases the involvement in crime for females but not for males (Simons \& Barr, 2014). Within correctional institutions, intimate relationships are proposed to contribute to the adjustment of incarceration in women. Wright, DeHart, KoonsWitt, and Crittenden (2013) found life history interviews with incarcerated women revealed that family members, children and intimate partners provided opportunities, motivation, social capital and support that can be a positive influence on the offender's behaviour.

The incarceration of female offenders disconnects them from relationships that are of valuable importance to them - family and children. Research suggests between $75 \%-80 \%$ of 
incarcerated women have children (Barrett, Allenby \& Taylor, 2010; Casey-Acevedo, Bakken \& Karle, 2004), and separation from family, particularly from children, was the most difficult experience (Collica, 2010). There is evidence that women with children have adverse experiences in adjusting to incarceration. Houck and Loper (2002) found that for incarcerated mothers, stress regarding competency as a parent was associated with increased anxiety, depression, and institutional misconducts. Women inmates receiving visits from their children were more likely to commit serious or violent misconducts or general rule infractions (Benning \& Lahm, 2016). Casey-Acevedo et al. (2004) suggest incarcerated mothers receiving visits from their children experience more difficulty adjusting to incarceration due to the emotional turmoil of separation from children. These findings support that relationships are imperative to the adjustment of incarceration for women offenders.

Despite the supporting evidence regarding of the importance of relationships, RCT has not been directly tested and more research that directly examines RCT in the adjustment to correctional institutions in female offenders specifically is needed. Further, the majority of these studies offered only correlational support, and did not test if these relationships predicted institutional adjustment.

Pathways Theory. In the early 1990s, feminist theorists examined feminist pathways, which underscores the linkages between victimization and offending (Miller \& Mullins, 2009). Feminist pathways theory argues that victimization in childhood leads to the likelihood that girls will run away from home and/or engage in drug use as a means to cope. Thus, criminal activities such as selling drugs, prostitution and robbery are a means to survive on the streets; and these criminal activities lead females to dangerous environments (Miller \& Mullins, 2009). 
Kathleen Daly's (1992) foundational research conceptualized feminist pathways theory concisely and found six distinct pathways. The Street woman experiences early trauma in her home environment, and runs away to escape abuse. The Harmed and harming woman experiences a history of trauma, and as a result may develop addictions as well as psychological issues. The Battered woman is in a relationship with an abusive male partner, and by defending herself becomes involved in the criminal justice system. The Drug-connected woman does not have a history of trauma, but becomes involved in selling or develops an addiction to drugs due to connections with an intimate partner or children. A Woman categorized as 'other' does not have a history of victimization, but is involved in crime due to self-involved purposes such as greed.

Research has consistently found that women offenders report higher incidences of lifetime trauma, emotional problems, and mental health concerns than men prisoners and women in the general population (DeHart, Lynch, Belknap, Dass-Brailsford, \& Green, 2014; Drapalski, Youman, Stuewig, \& Tangney, 2009; Fazel \& Seewald, 2012; Grella, Lovinger, \& Warda, 2013). Some studies found victimization is associated with recidivism (Herbst, BranscombBurgess, Gelaude, Seth, Parker \& Fogel, 2016; Taylor, 2015) and experiencing any trauma within the past year and history of physical abuse as an adult are significant predictors of recidivism (Bonta, Pang \& Wallace-Capretta, 1995; Scott, Grella, Dennis \& Funk, 2016). Conversely, other studies found victimization was not associated with recidivism (Anumba, Dematteo \& Heilbrun, 2012) and did not predict recidivism (Rettinger \& Andrews, 2010). Support for a relationship between victimization and institutional misconduct has also been implicated. Steiner and Wooldredge (2009) found in a sample of more than 2,200 women 
offenders, any abuse history was a significant predictor of violent and nonviolent institutional misconducts.

The pathways literature is criticized for placing too much emphasis on the victimization experiences of women. For example, Kruttschnitt (2016) notes that men also have histories of victimization. Some research found that despite the fact that victimization experiences are more prevalent in girl offenders, the relationship between victimization and offending is stronger for boys than girls (Asscher, van der Put, \& Stams, 2015; van der Put \& de Ruiter, 2016). Kruttschnitt (2016) suggests that the pathways approach assumes that a high prevalence of victimization is evidence of causation. High incidences of an event does not indicate causality, and without rigorous statistical support providing evidence for the predictive validity of victimization in female offenders, the assumption of causality cannot be inferred.

\section{Predictors of Institutional Misconduct in Offenders}

Research studies have found that age (Kolivoski \& Shook, 2016), prior institutional adjustment (Drury \& Delisi, 2010), and criminal history (Cihan, Davidson \& Sorensen, 2017) are strong predictors of institutional misconduct in men and women offenders. However, it should be noted that for the majority of these studies, the samples consisted of either predominately or only male offenders. Male gender (Kuanliang \& Sorensen, 2008), antisocial attitudes (ArbachLucioni, Martinez-García, \& Andrés-Pueyo, 2012), antisocial behaviour (Gendreau, Goggin \& Law, 1997), mental health issues (Kuanliang \& Sorensen, 2008), substance use/abuse problems (Arbach-Lucioni et al., 2012), and gang membership (Tasca, Griffin \& Rodriguez, 2010) have also been found to contribute to institutional misconducts.

\section{Predictors of Institutional Misconduct for Women Offenders}


Although limited, research has explored potential predictors of institutional misconduct by utilizing female-only offender samples. For instance, prior studies of female-only samples found age at first commitment, having a mental health disorder, offense type, gang affiliation, past violent criminal history, impulsivity, and callous and antisocial psychopathic traits predicted violent and nonviolent institutional misconducts (Blackburn \& Trulson, 2010; Thomson, Towl \& Centifanti, 2016). These predictors have been found to predict institutional misconducts in males as well (e.g., Arbach-Lucioni et al., 2012; Edens, Poythress, Lilienfeld, Patrick \& Test, 2008; Ruddell \& Gottschall, 2011).

Steiner and Wooldredge (2014) conducted a study to examine gender differences in the predictors of institutional misconduct in men and women offenders. Using multivariate analysis, they examined characteristics such as age, security classification, gang membership, and employment and incarceration experiences, such as number of visits, hours at a work assignment, and hours at recreational activities. For women offenders, security risk level was the only factor associated with violent misconduct. In contrast, men younger in age, classified as higher risk, and previously incarcerated were more likely to perpetuate violent misconduct. Men that spent more time at a facility work assignment, less time in recreation, and served less time incarcerated displayed less likelihood of perpetuating violent misconduct.

For nonviolent offences, age (young), race (black), security level (higher) and greater recreation time were attributed to greater likelihood of these offences in women. Men that were younger, black, unmarried, used drugs, had less than high school education, had extensive criminal history and gang members committed more nonviolent offences. Taken collectively, Steiner and Wooldredge (2014) suggest there are more similarities than differences in predicting 
misconduct in men and women. However, the authors advise the data is limited as they did not include gender-informed factors, which may be more relevant to women than men.

Gender-informed Predictors. The literature provides support for the utilization of gender-informed factors in predicting recidivism. Poverty (Holtfreter, Reisig \& Morash, 2004), dysfunctional relationships (Cobbina et al., 2012), exposure to trauma after incarceration (Scott et al., 2016), substance abuse (Taylor, 2008), and mental health issues (Becker, Kerig, Lim \& Ezechukwu, 2012) have been found to increase the risk of recidivism in female offenders. Van Voorhis et al. (2010) also contributed to the literature of gender-informed theory by examining if adding gender-informed variables to a gender-neutral assessment would yield incremental predictive validity to the prediction of institutional misconducts in women offenders.

A gender-informed "trailor" was created to predict institutional misconduct for three prison samples of women offenders in three American states (Colorado, Minnesota and Missouri). The gender-informed variables were added to the gender-neutral Level of Service Inventory-Revised (LSI-R; Andrews \& Bonta, 1995) for the Colorado and Minnesota samples. In Missouri, gender-informed variables were added to an accumulated gender-neutral scale. Gender-informed variables were assessed using two supplements. Supplement 1 consisted of measures of self-efficacy, adult victimization, self-esteem, relationship dysfunction, and child abuse; while Supplement 2 consisted of all Supplement 1 measures and additional measures such as symptoms of depression, symptoms of psychosis, educational strengths, family support, history of mental health concerns, housing safety, family conflict, relationship support, and anger/hostility. The gender-neutral assessment consisted of the subscales criminal history, financial situation, education/employment, accommodation, financial issues, companions, drug use/abuse, use of leisure time, attitudes/orientation, and emotional/mental health. It should also 
be noted that a scale measuring offence history was also included since that is currently used in the United States to determine classification of security levels (minimum, medium, and maximum) in women offenders.

Van Voorhis et al. (2010) examined the incremental predictive validity by using partial correlation analysis and predictive validity by using the area under the curve (AUC) statistic. Support was provided for the utilization of gender-informed factors in predicting institutional misconducts. Analysis demonstrated modest support for the predictive validity of the addition of the gender-informed scales as partial correlations demonstrated moderate to strong correlations and AUCs ranged from .62 to .70 . It should be noted that the scale measuring offence history had AUCs of .59 and .63 (one sample demonstrated non-significant associations with misconducts and was excluded from AUC analysis), while the gender-neutral scales demonstrated AUCs ranging from .58 to .68 . The scale measuring offence history and gender-neutral scales demonstrated lower AUCs than the addition of gender-informed scales. Overall, Van Voorhis et al. (2010) found support for the addition of gender-informed factors to gender-neutral assessments. The gender-informed variables that demonstrated promising results for predicting institutional misconduct included: self-efficacy, current mental health issues, anger/hostility, relationship dysfunction, child abuse, and family support.

Wright, Salisbury, and Van Voorhis (2007) also examined if the addition of genderinformed needs among 272 female offenders increase the predictive validity of institutional classification systems that are often used today. Number of misconducts and any misconducts were examined in six and twelve month periods of incarceration. The authors utilized genderinformed measures consisting of the items low self-esteem, low self-efficacy, childhood abuse, adult emotional abuse, adult physical abuse, adult harassment, low relationship support, high 
relationship conflict, high relationship dysfunction, parental stress, current depression/anxiety, and current psychosis. Gender-neutral measures were utilized and consisted of the items: antisocial attitudes, antisocial friends, low education, employment/financial difficulties, high family conflict, low family support, static substance abuse, dynamic substance abuse, history of mental illness, and anger control.

Wright et al. (2007) investigated if including gender-informed needs would increase the predictive validity of traditional institutional classification assessments. To do this, the authors created several measures. The Institutional Risk Scale was created to reflect custody assessment items used in the United States prison institutions, and summed six items: history of violence, prior felonies, prior violent offences, severity of current offence, history of escapes, prior incarcerations, and noncompliance from previous supervisions. The Gender-Neutral Needs scale summed six gender-neutral scales: Employment/Financial Difficulties, Mental Illness, Family Support, Family Conflict, Anger Control and Antisocial Attitudes. The Gender-Responsive Needs scale summed four gender-informed scales: Relationship Support, Psychosis, Depression/Anxiety, and Childhood Abuse.

They found the Gender-Responsive Needs scale and Gender-Neutral Needs scale had strong correlations to misconduct (correlation coefficients of gender-neutral needs ranged from $r$ $=.19$ to $r=.28, p<.01$ for all misconducts; while correlation coefficients of gender-informed needs ranged from $r=.25$ to $.28, p<.01$ for all misconducts). For the Gender-Neutral Needs scale, the gender-neutral factors that were associated with misconducts include: antisocial attitudes (correlation coefficients ranged from $r=.14, p<.05$ to $r=.18, p<.01$ for number of misconducts and any misconducts for 6-month and 12-month incarceration periods), high family conflict (correlation coefficients ranged from $r=.12, p<.05$ to $r=.19, p<.01$ for number of 
misconducts and any misconducts for 6-month and 12-month incarceration periods), low family support (correlation coefficients ranged from $r=.12, p<.05$ to $r=.20, p<.01$ for number of misconducts and any misconducts for 6-month and 12-month incarceration periods), and history of mental illness (correlation coefficients ranged from $r=.11, p<.05$ to $r=.19, p<.01$ for number of misconducts and any misconducts for 6-month and 12-month incarceration periods). Gender-informed need factors were also associated with misconducts, and included: childhood abuse (correlation coefficients ranged from $r=.20, p<.01$ to $r=.25, p<.01$ for number of misconducts and any misconducts for 6-month and 12-month incarceration periods), low relationship support (correlation coefficients ranged from $r=.13, p<.05$ to $r=.16, p<.01$ for number of misconducts and any misconducts for 6-month and 12-month incarceration periods), current depression/anxiety (correlation coefficients ranged from $r=.13, p<.05$ to $r=.23, p<$ .01 for number of misconducts and any misconducts for 6-month and 12-month incarceration periods), and current psychosis (correlation coefficients ranged from $r=.16, p<.01$ to $r=.31, p$ $<.01$ for number of misconducts and any misconducts for 6-month and 12-month incarceration periods).

Wright et al. (2007) found when gender-neutral and gender-informed needs were separately added to risk (the Institutional Risk Scale), correlations for the Gender-Neutral Needs scale combined with the Institutional Risk Scale were highly significant (correlation coefficients ranged from $r=.23$ to $r=.33, p<.01$ for number of misconducts and any misconducts for 6month and 12-month incarceration periods), and correlations for the Gender-Responsive Needs scale combined with the Institutional Risk Scale were also highly significant (correlation coefficients ranged from $r=.27$ to $r=.34, p<.01$ for number of misconducts and any misconducts for 6-month and 12-month incarceration periods). Finally, when the Gender- 
Responsive Needs scale and the Gender-Neutral Needs scale were combined, findings indicated the strongest, significant relationship with misconducts (correlations ranged from $\mathrm{r}=.28$ to $\mathrm{r}=$ .38 , all significant at $p<.01$ for number of misconducts and any misconducts for 6-month and 12-month incarceration periods). Thus, Wright et al. (2007) point out these findings provide evidence that both gender-neutral and gender-informed needs are imperative for consideration in predicting institutional misconduct.

Proposed Gender-informed predictors of institutional misconduct. The current study will elucidate gender-informed variables that can predict institutional misconduct and explore potential gender-informed variables that may provide incremental predictive validity to the CRS. The proposed gender-informed variables that will be utilized and rationale for the selection of these variables are outlined below.

Mental Health. The literature provides evidence that mental health issues are a common concern for women offenders (Derkzen, Booth, Taylor \& McConnell, 2013). Pathways Theory provides evidence that mental health is linked to women's developmental trajectory into crime (Gehring, 2016). Further, Davidson, Sorensen, and Reidy (2016) assessed the incremental predictive validity of the Personality Assessment Inventory (PAI) with general and assault misconduct in a sample of 2,000 female offenders. The PAI assesses psychopathology, and is used in corrections for assessing offenders. When incremental predictive validity was tested by including major PAI scales and background characteristics (e.g., age at first arrest, violent arrests) in a stepwise regression, the Paranoia (PAR) scale from the PAI and mental health need score at intake were significantly related to general misconduct. Further, PAR was a strong predictor of assaultive misconducts. This evidence guided the rationale for selecting mental health as a gender-informed predictor. 
Substance Abuse. The literature supports that substance abuse is an imperative factor for women offenders and crime (Swan \& Goodman-Delahunty, 2013). Substance abuse is implicated in Pathways Theory. The literature indicates substance abuse is a component to the gender-informed perspective of offending and is associated with the perpetuation of crime in women offenders (Bowles, DeHart \& Webb, 2012). Further, Davidson et al. (2016) found in a sample of 2,000 women offenders that the drug problems (DRU) scale from the PAI and substance abuse score at intake were significant predictors of general misconduct. The empirical support for substance abuse as a gender-informed factor guided its' inclusion in the study.

Relationship Dysfunction. The literature provides evidence that intimate relationships are potential factors for the perpetuation of crime in women (Jones, 2008; Stalans \& Lurigio, 2015). RCT and Pathways Theory provide support for the importance of relationships in women offenders. The literature regarding Pathways Theory indicates that dysfunctional relationships are an important component in the gendered pathway of the incarceration for women offenders (Hayes, 2015; Salisbury \& Van Voorhis, 2009). RCT also supports that relationship dysfunction has a strong influence for women's behaviour, as women grow and connect through relationships with others (Miller, 1976). Research regarding women offenders' relationship dysfunction and institutional misconduct is limited. Wright et al. (2007) and Van Voorhis et al. (2010) found some support for relationship dysfunction as a potential predictor.

Parental/Family Issues. Research provides evidence that some mothers with parental stress commit crimes for economic support (Ferraro \& Moe, 2003), and single mothers are more likely to recidivate than mothers with a partner (Bonta et al., 1995). RCT proposes family and parental issues as a potential factor for women offenders to commit crimes. Research relating to RCT supports parental relationships with children are important for institutional adjustment in 
women offenders (e.g., Houck \& Loper, 2002). Celinska and Sung (2014) found in a sample of men and women inmates ( $n=18,185,78.6 \%$ were male), the number of visits from friends and family was exclusively a negative predictor of prison rule violations in women. Further, Blanchette (2005) also found that prosocial family contact is an important negative predictor of institutional misconduct for federally sentenced women. However, research examining family relationships and the institutional misconduct in women offenders is limited. The evidence available provides some support for the inclusion of this factor.

Victimization. Feminist perspectives emphasize victimization in explaining crimes and perpetuation of criminal behaviours in women (Kruttschnitt, 2016). Further, Pathways Theory provides theoretical support for the inclusion of victimization as a gender-informed predictor of institutional misconducts in women offenders. Overall, support for victimization as a predictor of institutional misconduct is limited. Davidson et al. (2016) found the Traumatic Stress (ARD-T) scale from the PAI was a significant predictor of general misconduct in women offenders. However, other research found no support for victimization and institutional misconduct in women offenders (e.g., Wulf-Ludden, 2016). Due to the lack of research and some evidence supporting victimization as a predictor of institutional misconduct, inclusion of victimization was deemed appropriate for the current study.

Personal/Emotional. Variables such as anger (Verona \& Carbonell, 2000), self-efficacy (Van Voorhis et al., 2010), personal distress (Bonta \& Andrews, 2017), and self-esteem (Wright et al., 2007) can be included in this domain. Studies examining personal distress as a predictor of recidivism in offenders have found it is generally a weak predictor (Katsiyannis, Whitford, Zhang \& Gage, 2017). Van Voorhis et al. (2010) also found self-efficacy was negatively associated with recidivism. However, Rettinger and Andrews (2010) conducted a study with 531 
provincially sentenced women to determine predictors of recidivism. The authors found the Personal/Emotional subscale was positively related, but weakly, to general recidivism $(r=.14)$ and unrelated to violent recidivism $(r=.07)$. The emotional/personal factor is proposed as a gender-informed variable that demonstrates some evidence as a predictor of institutional misconduct in women offenders.

In summary, the review indicated that the CRS has been validated with women offenders (e.g., Barnum \& Gobeil, 2012); however, criticisms of the CRS include some studies lacking rigorous statistical analysis, AUC values that were less than .70, it was developed using a male sample, and it fails to consider gender-informed variables (for women). The theories reviewed provided explanations for institutional behaviours in women, and empirical evidence of these theories support that gender-informed factors can explain institutional behaviours. Further, the empirical data that was reviewed provided evidence that gender-informed factors can predict institutional misconduct in women. The research (e.g., Van Voorhis et al., 2010; Salisbury et al., 2009) suggested that when gender-informed variables are included in risk paradigms, the accuracy of tools predicting institutional misconduct increases. Thus, the current study will investigate if this also holds true for the CRS.

\section{Current Study}

The purpose of the current study is to investigate whether the inclusion of gender-informed factors can enhance the prediction of institutional misconduct in federally sentenced women offenders; including Indigenous women offenders. The identified gender-informed domains include: mental health, substance abuse, relationship dysfunction, parental/family issues, victimization, and personal/emotional factors. These domains will be tested to determine if they provide incremental predictive validity to the CRS. The literature in regards to gender-informed 
variables in predicting institutional misconducts is insufficient; the scarcity of research is an impediment to making robust inferences from the data. The current study will contribute to the gap in this literature to allow for stronger inferences regarding the inclusion of gender-informed variables in risk assessment paradigms.

Gender-informed studies have been criticized for the lack of rigorous methodological research designs by utilizing partial correlational analysis to determine incremental predictive validity (e.g., Van Voorhis et al., 2010). The current study will address this issue, as a more rigorous statistical analysis, cox regression survival analysis, will be utilized to determine which gender-informed factors are predictive of institutional misconducts. Incremental predictive validity will then be determined by a series of ROC comparisons and hierarchal cox regression analysis. To date, no study has examined the incremental predictive validity of these genderinformed variables utilizing a statistically robust procedure.

The majority of classification systems are gender-neutral (e.g., Brennan \& Austin, 1997; Salisbury, Van Voorhis \& Spiropoulos, 2009), as they have been developed and validated predominately with male offenders. The CRS is no exception, as it has been developed and normed on male offenders (Luciani et al., 1996). This has lead some to question the extent of the applicability of the CRS to women offenders (OAG, 2017), and warrants investigation if the CRS can be improved for the assessment of women offenders. If additional factors can be incorporated into these assessments and validated, the efficiency of these assessments can be enhanced. The current study will elucidate if the addition of gender-informed factors enhance the accuracy of predicting institutional misconduct in women and Indigenous women.

Research Question 1: Which gender-informed variables that are proposed can predict institutional misconduct? 
Hypothesis: It is hypothesized the gender-informed domains: mental health, substance abuse, relationship dysfunction, parental/family issues, victimization, and personal/emotional factors will be predictive of institutional misconducts. The gender-informed domains mental health (Davidson et al., 2016), substance abuse (Davidson et al., 2016), relationship dysfunction (Wright et al., 2009), parental issues (Celinska \& Sung 2014), and victimization (Davidson et al., 2016) have been found to be significant predictors of institutional misconducts. As such, support for these variables is implicated in the literature. Since the personal/emotional domain has been implicated as a weak predictor of misconduct (Rettinger \& Andrews, 2010), it is hypothesized this construct will be a weak predictor of misconduct. Due to the absence of literature regarding gender-informed predictors for Indigenous women offenders, no hypothesis pertaining to Indigenous women offenders in this sample is proposed.

Research Question 2: If gender-informed variables do emerge, can they yield incremental predictive validity above and beyond the Custody Rating Scale?

Hypothesis: It is hypothesized the gender-informed domains will provide incremental predictive validity above and beyond the Custody Rating Scale. Previous research (e.g., Van Voorhis et al., 2010; Wright et al., 2007) has found that the addition of gender-informed variables to gender-neutral assessments increases the accuracy of tools predicting institutional misconducts. Further, Rubenfeld (2014) suggests it is worthwhile for future research to explore the addition of gender-informed variables to the CRS that may best predict incarceration behaviours. Since a reweighted CRS did demonstrate some improvement over the CRS in Rubenfeld's (2014) study, it is possible the addition of gender-informed variables will create a more suitable measure for women offenders. Gender-informed variables capture the gendered context of women's lives, and this may allow for additional accuracy to the risks/needs that 
women offenders encounter in institutional settings. Due to the absence of prior literature concerning the addition of gender-informed variables to gender-neutral assessments for Indigenous women offenders specifically, no hypothesis is proposed.

\section{Method}

\section{Participants}

The sample was derived from an archival database provided by Correctional Service of Canada. The population of all federally sentenced women admitted to custody between September 28, 2009 to January 8, 2017, and who had a valid and complete Dynamic Factors Identification and Analysis-Revised (DFIA-R) assessment were included in this study. ${ }^{1}$ This study comprised of 1,555 women with new warrant of committal admissions, with the average age at admission being 34.73 years $(S D=10.72)$, range $=17$ to 78 . Of the 1,555 women offenders, 577 (37.1\%) were Indigenous, while 971 (62.4\%) were non-Indigenous. The ethnic backgrounds of the remaining seven women offenders were unknown $(0.5 \%)$.

The majority of the sample was admitted to the Prairies region (34.4\%), while $29.0 \%$ were admitted to the Ontario region, $14.0 \%$ to the Atlantic region, $13.5 \%$ to the Quebec region, and $9.1 \%$ to the Pacific region. The vast majority of women offenders in the sample were also serving their first federal sentence $(N=1296,83.3 \%)$, while the remaining women offenders in the sample were serving their second or more federal sentence $(N=259,16.7 \%)$.

\section{Procedure}

The data that was utilized for this study was gathered from the Offender Management Systems (OMS), which is a database case management file that is used by the Correctional

\footnotetext{
${ }^{1}$ About $20 \%$ of women federal offenders do not complete a comprehensive OIA. These women will not be included in the sample due to the unavailability of data.
} 
Service of Canada and the Parole Board of Canada. OMS provided information regarding demographic factors, level of risk and need, and institutional misconducts.

The dataset was stripped of all identifying information (e.g., names, offender IDs, sentence IDs, birthdates). To replace the offender ID, a participant ID was created that is not associated with the offender within the Offender Management System (OMS). The Research Branch of Correctional Service of Canada has the master key that links the participant IDs to the identifying information. Correctional Service of Canada gave the de-identified data to the researcher via an email as an encrypted excel document. This study was accepted via two independent research ethics boards (REBs): Carleton University REB and Correctional Service of Canada REB.

Correctional Service of Canada provided the data and gender-responsive variables were identified from the Offender Intake Assessment (OIA). These variables were based on measures that assess women's risk and needs and a review of the literature was also utilized as a guide. This information was collected via a data drop from the Offender Management System (OMS) warehouse to ensure that there was a sufficient number of women offender participants.

The outcome data (minor and serious misconducts charges) were dropped from OMS. Institutional charges are behaviours that result in formal charges within the correctional institution and are tracked in the OMS database. Correctional staff utilize their own discretion in accordance to Commissioner's Directive 580 (Correctional Service of Canada, 2015) to classify charges as either minor or major. Major charges result in more formal processing than minor charges. An example of a major charge can include an inmate fight or possession of drugs, while minor charges can include disobeying an order (Grant, Varis \& Lefebvre, 2005).

\section{Measures and Materials}


The data utilized for this study was gathered from The Offender Management System (OMS), de-identified, and provided by CSC. Information from OMS is utilized to manage information regarding federal offenders for the duration of their sentences. The system collects, stores and retrieves imperative information for tracking offenders and creating decisions for the cases of offenders. This data is associated with demographic information from the offender, level of risk and need, and institutional misconducts.

\section{CoMHISS Information}

At intake, the Computerized Mental Health Intake Screening System (CoMHISS) is stored on the Health Care file and is administered to all federal offenders (Correctional Service of Canada, 2007; 2014). CoMHISS is a screening process for mental health that is composed of a computerized psychometric test battery that measures indicators of mental health and is an offender self-administered assessment tool that specifically assesses mental health needs. It is completed within 3 to 14 days of admission and is used to identify offenders who are experiencing any mental health symptoms that may require further assessment and intervention. The assessment includes questions related to past or present mental health symptoms, diagnoses, medications or treatments, suicidal ideations, attention deficit hyperactivity disorder (ADHD), as well as cognitive deficiencies and intellectual abilities. The mental health indicators CoMHISS measures include, but are not limited to, depression, anxiety, psychotic disorders, suicidal ideation, and obsessive compulsive disorders. Once the offender completes these battery of tests, an official report will be generated to be placed in the offender's confidential Psychology file. CoMHISS consists of 132 questions, and includes item and validated measures assessing: historical and current psychological functioning, current depression and suicide risk, psychological/psychiatric symptoms, and cognitive impairment. These measures include: 
a) The Depression, Hopelessness and Suicide Screening Form (DHS, Mills \& Kroner, 2004; 39 items) - items are answered dichotomously (True or False).

b) The Brief Symptom Inventory (BSI, Derogatis, 1993; 53 items) - items are rated on a scale from 0 (not at all) to 4 (extremely).

c) General Ability Measure for Adults (GAMA, Naglieri \& Bardosa, 1997; 66 items) - consists of entirely nonverbal abstract designs with 6 response options that includes four subscales: Matching, Analogies, Sequences and Construction.

d) Adult Self-Report Scale for attention deficit hyperactivity disorder (ASRS, Kessler et al., 2006; 18 items) - items are rated on a 5-point Likert scale with the first six items corresponding directly to the DSM-IV (American Psychiatric Association, 2000) criteria for ADHD.

CoMHISS has been found to be a valid instrument to screen for mental health concerns in male offender populations. Stewart, Wilton and Malek (2011) compared CoMHISS scores to a mental health diagnosis by licensed mental health professionals and found an overall accuracy rate of $58.2 \%$. The authors found $40 \%$ of the total sample were false positives (indicated a mental health concern is present when it is not). For women offenders, Archambault, Stewart, Wilton, and Cousineau (2010) indicate that DHS measure (depression, hopelessness, current suicidal ideation, and historical suicide) has been validated for federally sentenced women and specific norms have been established. Women that had higher CoMHISS scores were more likely to have drug and alcohol abuse problems and greater risk and need ratings than women that had low scores on the CoMHISS (Archambault et al., 2010). 
Data from the OMS includes information taken from the Offender Intake Assessment (OIA, Motiuk, 1997) database. Two main components comprise the OIA: Criminal Risk Assessment (CRA) and the Dynamic Factors Identification and Analysis (DFIA-R; Brown \& Motiuk, 2005). The Criminal Risk Assessment comprises the offender's Criminal Risk Level, which is rated as either low, medium or high. The CRA is based on the following components: criminal history records, which includes the number and type of prior and current offences (including as an adult and youth); offence severity index, which include current or prior convictions characterized by the offence type, degree of force, sentence length, and extent of physical/mental harm caused; sex offence history checklist, which comprises any current or prior sex or sex-related offences, information of victim, treatment history and serious harm assessments; review of detention criteria, which includes legislated criteria utilized to prevent the release of Dangerous Offenders.

The DFIA-R utilizes a similar approach to the CRA, and examines offenders' needs based on seven domains: employment, family/marital, associates/social interaction, substance abuse, community functioning, personal/emotional orientation, and attitude. For each criminogenic need domain, case managers utilize a list of indicators (dynamic risk factors) and rate the severity of each need. A summary report is then completed that helps to assess the overall risk/needs of an offender that ranges from low risk, low need to high risk, high need. This comprehensive assessment is the basis for the offender's correctional plan. Further, each domain is comprised of a series of yes/no indicators that help determine the overall domain rating. For the current study, these indicators were used to measure the gender-informed constructs Mental Health, Substance Abuse, Relationship Dysfunction, Parental/Family Issues, Victimization, and 
Personal/Emotional. The specific indicators that were used for each construct is presented in Table 1 later in the document.

The Dynamic Factor Identification and Analysis-Revised:

The Dynamic Factor Identification and Analysis-Revised (DFIA-R) is a component of the Offender Intake Assessment. The DFIA-Revised was implemented in September 2009 and includes the seven 'need' domains. Utilizing information from case files, interviews with offenders, and responses from staff, offenders are assessed in accordance to seven domains: Employment/Education, Attitude, Family/Marital, Substance Abuse, Community Functioning, Substance Abuse, and Personal/Emotional. The DFIA-R provides several key functions for the assessment of offenders: it establishes a profile of offender's needs for the population in order to guide the focus of correctional interventions within the entire service; acts as a case management tool to provide the priority needs of the offender, which forms the foundation of the offender's correctional plan; and establishes an estimate of the dynamic risk of an offender likelihood of recidivism and estimates the overall risk rating (Brown \& Motiuk, 2005).

In a sample of 4,798 federally sentenced male and 1,368 female offenders Stewart, Wardrop, Wilton, Thompson, Derksen, and Motiuk (2017) found support for the DFIA-R as demonstrating good validity and good internal consistency (an indicator of reliability). Specifically, Cronbach's $\alpha$ values for the overall DFIA-R needs ratings revealed very high internal consistency (Cronbach's $\alpha=.95$ for males; Cronbach's $\alpha=.95$ for females). Cronbach's $\alpha$ for each domain ranged from .74 to .93 for males and ranged from .71 to .94 for females. Further, the tool demonstrated to be a valid measure of dynamic risk for the offender groups, as offenders that scored higher on the need ratings had poorer community outcomes. Further, it was found that the overall DFIA-R need ratings were predictive of revocations and revocations with 
an offence (Harrell's C ranged from .54 to .61 for revocations and .55 to .60 for revocations with an offence). Thus, Stewart et al. (2017) concluded that the DFIA-R is a valid assessment for predicting release outcomes across offender groups.

\section{W-CASA Information}

The Women's Computerized Assessment of Substance Abuse (W-CASA) was developed to assess substance abuse issues in women offenders upon admission into federal custody and was implemented in 2011. The W-CASA collects gender-specific information regarding pregnancy, substance abuse problems, and relationships. The W-CASA is administered as part of the intake assessment process, and all federally sentenced women entering the institutions are referred to the W-CASA. The W-CASA is a 261-item computerized assessment that examines substance abuse in the following areas:

a) History of Alcohol Use (16 items)

b) Alcohol Use and Life Areas (4 items)

c) Dependence/Consequences (PRD; 15 items)

d) Severity - Alcohol Dependence Scale (ADS, Skinner \& Horn; 25 items)

e) History of Drug Use (22 items)

f) Prescription Drug Use (10 items)

g) Polysubstance Abuse (4 items)

h) Drug Use and Life Areas (4 items)

i) Severity/Consequence - Drug Abuse Screening Test (DAST, Skinner, 1982; 20 items )

j) Psychological Dependence - Severity of Dependence Scale (SDS, Gossop et al., 1995; 5 items) 
k) Current Perception (5 items)

1) Crime and Substance Use (13 items)

m) Relationships (19 items)

n) Substance Use and Pregnancy (17 items)

o) Previous Programming (12 items)

p) Readiness to Change (RTC; 15 items)

q) Respondent Satisfaction (12 items)

The W-CASA includes two standardized measures that are included in the current study: the ADS (Skinner \& Horn, 1984) and DAST (Skinner, 1982). The ADS is a 25-item assessment tool that determines the degree of physiological dependence to alcohol. The DAST is a 20-item assessment tool that examines the abuse of drugs other than alcohol. Both scales consist of overall ratings of none, low, moderate, substantial or severe. Both of the scales demonstrate acceptable psychometric properties as the ADS has been found to have a Cronbach's alpha of .91 (Skinner \& Horn, 1984) and the DAST has been found to have Cronbach's alpha ranging from .74 to .92 (Skinner, 1982; Yudko, Lozhkina \& Fouts, 2007). It should be noted that the Computerized Assessment of Substance Abuse (CASA) assesses the seriousness and nature of male offender's substance abuse as well, and also includes the ADS and DAST.

Despite that no formal validation or reliability study has been completed to date with the W-CASA, prior research has indicated the W-CASA to be an important factor to consider with institutional behaviours in women offenders. MacDonald, Gobeil, Biro, Ritchie and Curno (2015) conducted a study utilizing the W-CASA to examine the relationships of substance abuse severity and type of user with institutional and post-release behaviours in federal women offenders. The authors found that women with substantial or severe substance use issues were 
5.2 and 6.6 more likely, respectively, to have a disciplinary offence. Further, women with substantial or severe substance use issues were 5.6 and 7.0 times more likely, respectively, to be placed into segregation. The authors also found that women that did not demonstrate substance abuse issues or a low severity were more likely to be granted discretionary release and less likely to return to custody.

Other research has also found that having a W-CASA recommendation for substance abuse treatment at intake was associated with return to custody for both Indigenous and nonIndigenous women offenders (Thompson, Forrester \& Stewart, 2015). Further, a study examining the impact of Section 84 releases on post-release success for Indigenous offenders found the W-CASA treatment need was one of the stronger covariates that remained in the survival analysis model investigating revocations (Correctional Service of Canada, 2018b). These findings provide support for the link between the W-CASA and behavioural outcomes in women federal offenders, such as return to custody and institutional behaviours.

\section{Custody Rating Scale:}

The Custody Rating Scale (CRS) is utilized to assist with the initial security classification of offenders. The CRS is composed of two independently scored sub-scales: the Institutional Adjustment scale which consists of five items, and Security Risk scale which consists of seven items. The scale's recommended security classification is based on the total score of the subscales, and for each incident that occurs item scores will increase. As scores increase for each sub-scale, higher security classifications are warranted. Scores range from 0 to 186 points on the Institutional Adjustment scale, while scores range from 17 to 190 points on the Security Risk scale. The cut-off scores for the CRS is as follows: 
a) Minimum security: 0 to 85 on the Institutional Adjustment scale and 0 to 63 on the Security Risk scale

b) Medium security: between 86 to 94 on the Institutional Adjustment scale and between 0 to 133 on the Security Risk scale; or between 0 to 85 on the Institutional Adjustment scale and 64 to 133 on the Security Risk scale

c) Maximum security: 95 or greater on the Institutional Adjustment scale or 134 or greater on the Security Risk scale

Researchers have found the CRS demonstrates good support for reliability of the Institutional Adjustment scale and fair support for the Security Risk scale (Luciani et al., 1996); as well as good predictive validity (Grant \& Luciani, 1998; Luciani et al. 1996). Further, Blanchette et al. (2002) found the Institutional Adjustment scale of the CRS demonstrated strong predictive validity of both violent and general institutional misconducts in Indigenous and non-Indigenous women offenders. The Security Risk scale of the CRS also demonstrated predictive validity for general and violent misconducts in non-Indigenous women offenders (Blanchette et al., 2002). Thus, the CRS has demonstrated adequate validity for women offenders.

\section{Gender-informed variables:}

The gender-informed variables were based on the combination of the measures from the OMS data (e.g., DFIA-R, CoMHISS, W-CASA) and as guided by the literature. Information from this OMS data were used to determine which items correspond to the proposed genderinformed domains. Table 1 below presents all the proposed gender-informed variables with each individual item listed alongside the corresponding measure the item was derived from. 
Table 1

Proposed Gender-informed Predictors

\begin{tabular}{|c|c|c|}
\hline $\begin{array}{c}\text { Gender-informed } \\
\text { Domain }\end{array}$ & Measure & Items \\
\hline Mental Health & $\begin{array}{l}\text { CoMHISS } \\
\text { Information }\end{array}$ & $\begin{array}{l}\text { Brief Symptom Inventory Global Severity Index } \\
\text { Depression, Hopelessness and Suicide Screening Form } \\
\text { depression } \\
\text { Depression, Hopelessness and Suicide Screening Form } \\
\text { hopelessness } \\
\text { Depression, Hopelessness and Suicide Screening Form } \\
\text { current suicidal ideation } \\
\text { Depression, Hopelessness and Suicide Screening Form } \\
\text { cognitive suicide } \\
\text { Depression, Hopelessness and Suicide Screening Form } \\
\text { historical suicide } \\
\text { Mental Health Indicator question } 1 \text { : Have you ever been } \\
\text { assessed for a mental illness or mental disorder? } \\
\text { Mental Health Indicator question } 2 \text { : Have you been } \\
\text { diagnosed in the past with a mental illness or mental } \\
\text { disorder? } \\
\text { Mental Health Indicator question 3: Do you currently } \\
\text { have a mental health problem and/or illness? } \\
\text { Mental Health Indicator question } 4: \text { Are you currently } \\
\text { diagnosed with a mental illness or disorder? } \\
\text { Mental Health Indicator question 5: Have you ever } \\
\text { received treatment for a mental illness or disorder? } \\
\text { Mental Health Indicator question } 6 \text { : Have you been under } \\
\text { the care of a health professional for a mental health } \\
\text { problem and/or mental illness in the last } 6 \text { months? } \\
\text { Mental Health Indicator question } 7 \text { : Have you ever taken } \\
\text { medication in the past for a mental illness or disorder? } \\
\text { Mental Health Indicator question } 8 \text { : Have you been } \\
\text { prescribed medication for a mental health problem and/or } \\
\text { mental illness in the last } 6 \text { months? } \\
\text { Mental Health Indicator question 9: Do you currently take } \\
\text { medication for a mental illness or disorder? }\end{array}$ \\
\hline
\end{tabular}

(Continued) 


\begin{tabular}{|c|c|c|}
\hline $\begin{array}{c}\text { Gender-informed } \\
\text { Domain }\end{array}$ & Measure & Items \\
\hline & $\begin{array}{l}\text { CoMHISS } \\
\text { Information }\end{array}$ & $\begin{array}{l}\text { Mental Health Indicator question 10: Have you ever } \\
\text { been hospitalized in the last for a mental illness or } \\
\text { disorder? } \\
\text { Mental Health Indicator question 11: Were you } \\
\text { hospitalized for a mental illness or disorder in the last } \\
\text { month before you were incarcerated? } \\
\text { Mental Health Indicator question 12: Have you } \\
\text { intentionally injured yourself without trying to kill } \\
\text { yourself? } \\
\text { General Ability Measure for Adults (GAMA) } \\
\text { Adult Self-Report Scale for attention deficit } \\
\text { hyperactivity disorder (ASRS) }\end{array}$ \\
\hline Substance Abuse & $\begin{array}{l}\text { DFIA-R } \\
\text { Substance } \\
\text { Abuse Domain }\end{array}$ & $\begin{array}{l}\text { SUB01: Early age alcohol use } \\
\text { SUB02: Frequently engages in binge drinking } \\
\text { SUB03: Has combined the use of alcohol and drugs } \\
\text { SUB04: Alcohol use interferes with employment } \\
\text { SUB05: Alcohol use interferes with interpersonal } \\
\text { relationships } \\
\text { SUB06: Alcohol use interferes with physical or } \\
\text { emotional wellbeing } \\
\text { SUB07: Excessive alcohol use is part of the offender's } \\
\text { lifestyle } \\
\text { SUB08: Early age drug use } \\
\text { SUB09: Has gone on drug-taking bouts or binges } \\
\text { SUB10: Has combined the use of different drugs } \\
\text { SUB11: Drug use interferes with employment } \\
\text { SUB12: Drug use interfere with interpersonal } \\
\text { relationships } \\
\text { SUB13: Drug use interferes with physical or emotional } \\
\text { well-being } \\
\text { SUB14: Regular drug use is part of the offender's } \\
\text { lifestyle } \\
\text { SUB15:Alcohol or drug use has resulted in law } \\
\text { violations } \\
\text { SUB16: Becomes violent when drinking or using drugs } \\
\text { SUB17:Alcohol and/or drug use is part of the offence } \\
\text { cycle } \\
\text { SUB18: Previously referred to programs }\end{array}$ \\
\hline
\end{tabular}

(Continued) 


\begin{tabular}{|c|c|c|}
\hline $\begin{array}{l}\text { Gender-informed } \\
\text { variable }\end{array}$ & Measure & Items \\
\hline & $\begin{array}{l}\text { W-CASA } \\
\text { Information }\end{array}$ & $\begin{array}{l}\text { Alcohol Dependence Scale score (ADS) } \\
\text { Drug Abuse Screening Test score (DAST) }\end{array}$ \\
\hline $\begin{array}{l}\text { Relationship } \\
\text { Dysfunction }\end{array}$ & $\begin{array}{c}\text { DFIA-R } \\
\text { Marital/Family }\end{array}$ & $\begin{array}{l}\text { FAM06: Inability to maintain enduring intimate } \\
\text { relationship } \\
\text { FAM07: Intimate relationships problematic } \\
\text { FAM09: Perpetuated spousal violence } \\
\text { Marital Status }\end{array}$ \\
\hline $\begin{array}{l}\text { Parental/Family } \\
\text { Issues }\end{array}$ & $\begin{array}{c}\text { DFIA-R } \\
\text { Marital/Family }\end{array}$ & $\begin{array}{l}\text { FAM 02:Relationships with parental figure were } \\
\text { negative during childhood } \\
\text { FAM05: Family members criminally active during } \\
\text { childhood } \\
\text { FAM12: Has significant difficulties handling parenting } \\
\text { responsibilities }\end{array}$ \\
\hline & $\begin{array}{l}\text { DFIA-R } \\
\text { Associates }\end{array}$ & $\begin{array}{l}\text { ASS09: Prosocial family support is limited } \\
\text { ASS10: Prosocial support from friends is limited }\end{array}$ \\
\hline Victimization & $\begin{array}{c}\text { DFIA-R } \\
\text { Marital/Family }\end{array}$ & $\begin{array}{l}\text { FAM01: Limited attachment to family unit during } \\
\text { childhood } \\
\text { FAM03: Abused during childhood } \\
\text { FAM04: Witnessed family violence during childhood } \\
\text { FAM 08: Victimized by spousal abuse }\end{array}$ \\
\hline Personal/Emotional & $\begin{array}{c}\text { DFIA-R } \\
\text { Personal/ } \\
\text { Emotional }\end{array}$ & $\begin{array}{l}\text { PER05: Has difficulty coping with stress } \\
\text { PER06: Gives up easily when challenged } \\
\text { PER13: Assertiveness skills are limited } \\
\text { PER15: Has difficulty solving interpersonal problems } \\
\text { PER18: Frequently feels intense anger } \\
\text { PER19: Frequently supresses anger } \\
\text { PER21: Has low frustration tolerance }\end{array}$ \\
\hline
\end{tabular}

\section{Institutional Misconduct:}

Institutional misconduct was also assessed utilizing the OMS database. Reports from correctional staff provide information regarding the incidents of institutional misconduct, which include the nature of the misconduct, the offender's role (victim, perpetrator, or other), if 
weapons were used during the misconduct, and the extent of harm inflicted. However, for the current study institutional misconducts consisted of an offender receiving an institutional charge for either a minor or serious misconduct. Institutional charges include information such as who was charged by whom, description of event, location of event, outcome of the hearing related to the charge, and only includes offenders that were perpetrators. As cited in Kroner and Mills (2001), minor misconducts include activities such as disrespectful behaviours towards an officer, improper dress, failure to attend an institutional count, and noncompliance to directions. Major misconducts include substance use, instigating a riot, uttering threats, assaults, refusing a direct order, and refusing urinalysis. As such, receiving a charge either for a minor or serious misconduct was used in the study to examine if an offender received any institutional charge while incarcerated. For offenders that received both a minor or serious charge during the duration of incarceration in the study, the incident that occurred first (either a minor or serious charge) was utilized to determine the occurrence of any institutional charges in this sample of women offenders.

\section{Statistical Approach}

Research question 1. All analyses to address research question 1 were tested using SPSS version 25. The first research question asks which gender-informed predictors will emerge to predict any misconduct charges. To test this, the current study utilized three measures (DFIA-R, CoMHISS, and W-CASA) to select, based on prior literature and theory, all gender-informed predictors. A total of 60 potential gender-informed predictors (presented in Table 4) were selected based on this criteria. The gender-informed predictors were then tested utilizing a series of univariate survival analyses to determine which gender-informed predictors would emerge as predictive for any misconduct charges. 
Research question 2. The gender-informed factors that emerged as predictive from the survival analysis were then retained (i.e., 17 number of variables were found to be individually significant $-p<.05)$. The individual gender-informed factors that were found to be predictive were then summed to create gender-informed scales. More information of how the genderinformed scales were created is described below. To determine incremental predictive validity of the gender-informed scales, AUC comparisons were computed and a hierarchal cox regression was also computed. All AUC comparisons were computed in MedCalc Version 18.9 and NCSS version 12 by utilizing the method of Hanley and McNeil (1983), and the hierarchal cox regression analysis was computed using SPSS version 25.

\section{Results}

\section{Data Screening}

Missing data, outliers, influential cases, and assumptions. A missing value analysis (MVA) was conducted to determine missing data on the following measures: Institutional Adjustment scale; Security Risk scale; GSI subscale of the Brief Symptom Inventory; DHS Depression; DHS Hopelessness; DHS Cognitive Suicide; DHS Current Ideation; DHS Historical Suicide; Mental Health Indicators; General Ability Measure for Adults (GAMA); Adult SelfReport Scale for attention deficit hyperactivity disorder (ASRS); the DFIA-R Substance Abuse domain items; the DFIA-R Family/Marital domain items; the DFIA-R Associates domain items; the DFIA-R Personal/Emotional domain; marital status; minor misconduct charges; major misconduct charges; date of first minor charge; date of first major charge; admission date; and date institutional follow-up ended.

The pattern of missing data ranged from $0 \%$ for minor misconduct, major misconduct, admission date, and date institutional follow-up ended to $76.8 \%$ for certain Mental Health 
Indicators; specifically, the following variables had missing data exceeding 10\%: General Ability Measure for Adults (GAMA; $62.8 \%$ of missing data), and Adult Self-Report Scale for attention deficit hyperactivity disorder (ASRS; $62.5 \%$ of missing data), and consequently were dropped from all subsequent analyses. Many missing data values were also observed, albeit not as problematic as the aforementioned variables, for the Global Severity Index subscale of the BSI (27.9\%); DHS Depression (28.0\%); DHS Hopelessness (27.8\%); DHS Cognitive Suicide (27.7\%); DHS Current Ideation (27.6\%); DHS Historical Suicide (27.7\%), and DFIA-R PER06 item 'gives up easily when challenged' $(10.8 \%)$. Due to the theoretical importance of these variables for the gender-informed mental health domain, these items were retained but the pattern of missing data for these variables was examined more closely.

The pattern of missing data was examined with a series of t-tests and chi-square analysis for variables missing over $10 \%$ of data and all other variables. The Global Severity Index subscale of the BSI, DHS Depression, DHS Hopelessness, DHS Cognitive Suicide, DHS Current Ideation, DHS Historical Suicide, and the DFIA-R PER06 'Gives up easily when challenged' were compared to all DFIA-R predictors, date CoMHISS was administered, date DFIA-R was administered, date W-CASA was administered, version number of CoMHISS, age at admission, any misconduct, marital status and Indigenous status. Results from the t-tests indicated (after applying the Bonferonni correction $p=.05 / 51$ of comparisons $=p<.001$ ) significant patterns emerged between the Global Severity Index subscale of the BSI with the actual admission date, DFIA-R Substance Abuse predictors, date DFIA-R was administered and date W-CASA was administered. Offenders with missing data on the Global Severity Index subscale of the BSI had earlier admission dates, earlier DFIA-R admission dates, earlier W-CASA admission dates and greater DFIA-R Substance Abuse scores than offenders without missing data on the Global 
Severity Index subscale of the BSI. The DHS Indicator items had significant patterns that emerged with the actual admission date, DFIA-R Substance Abuse predictors, date CoMHISS was administered, date DFIA-R was administered and date W-CASA was administered. Offenders with missing data on the DHS Indicators had earlier admission dates, earlier DFIA-R admission dates, earlier W-CASA admission dates, later CoMHISS admission dates, and greater DFIA-R Substance Abuse scores than offenders without missing data on the DHS Indicators. Finally, the DFIA-R PER06 'gives up easily when challenged' item demonstrated some significant patterns with the Personal/Emotional predictors of the DFIA-R. Offenders with missing data on the DFIA-R PER 06 'gives up easily when challenged' item had greater scores on the Personal/Emotional predictors of the DFIA-R than offenders without missing data on the DFIA-R PER 06 item.

Chi-square tests from the missing data analysis also revealed significant findings. Significant patterns emerged between the Global Severity Index subscale of the BSI, DHS Depression, DHS Hopelessness, DHS Cognitive Suicide, DHS Current Ideation, and DHS Historical Suicide with Indigenous status. Indigenous women were less likely to have scores on these variables than non-Indigenous women. Explanation for these findings are discussed in the Limitations section below. Notwithstanding the observation that Indigenous women were more likely to have more missing data than non-Indigenous women, these variables will still be examined given their theoretical importance. In sum, the missing value analysis suggests that the data is most likely missing at random (MAR) and thus pairwise deletion and listwise deletion will be deal with missing data (Tabachnick \& Fiddell, 2013).

Standardized z-scores greater than $-/+3.25$ and box plots were examined for univariate outliers. Univariate outliers were found for the GSI subscale of the Brief Symptom Inventory, 
DHS Current Suicidal Ideation, DHS Cognitive Indicator, Security Risk Score and Institutional Adjustment score. These variables were candidates for transformation. Bivariate scatterplots, histograms, box-plots and normal q-q plots were also examined to check for heteroscedasticity, normality and linearity. Variables that indicated non-normality and heteroscedasticity include ADS, GSI subscale of the Brief Symptom Inventory, DHS Depression, DHS Current Ideation subscale, DHS Hopelessness subscale, DHS Cognitive Indicator subscale, DHS Historical Suicide subscale, Institutional Adjustment score, and Security Risk score. Thus, these variables were candidates for transformations.

Based on Tabachnick and Fiddell (2013), the ADS was transformed using the logarithm method, GSI subscale of the Brief Symptom Inventory was transformed using the square root method, DHS Depression was transformed using the square root method, DHS Hopelessness subscale was transformed using the logarithm method, DHS Historical Suicide subscale was transformed using the square root method, Institutional Adjustment score was transformed using the square root method and Security Risk score was transformed using the logarithm method. DHS Current Suicidal Ideation and DHS Cognitive Indicator subscales were dichotomized. Survival analyses were run using both transformed and non-transformed data. Fewer items were indicated as being predictive when the transformed ADS was run in the analysis in comparison to when the ADS was not transformed. No substantial differences were noted among the remaining transformed variables. Thus, the ADS was log transformed while the remaining variables were not transformed. Dichotomous variables were also analyzed for extreme uneven splits, as dichotomous variables with 90-10 splits are considered problematic (Tabachnick \& Fiddell, 2013). Frequency distributions were examined for extreme uneven splits and no extreme uneven splits were evident. All dichotomous variables in the analysis were retained. 
Multivariate outliers were tested using Mahalanobis Distance. The values of the Mahalanobis Distance were compared with the critical value listed in the $\chi^{2}$ distribution $(d f=50$, $p=.001)$. Using a critical value of $86.661,27$ multivariate outliers were identified. To examine possible influential cases at the multivariate level, Cook's Distance, Leverage, and DFFIT values were computed. Using the appropriate criterion for each test (Cook's Distance > 1.00; DFFIT > $+/-1.00$; Leverage $>2 p / n)$, cases identified on two or more of these indexes were considered influential. There were 27 cases identified as multivariate outliers, and survival analysis was rerun with the 27 multivariate outliers removed. The predictors FAM04 'witnessed family violence during childhood,' SUB17 'alcohol and/or drug use is part of the offence cycle,' and PER06 'gives up easily when challenged' changed to non-significance; thus the 27 multivariate outliers were removed, with a new total $N=1528$.

A correlation matrix was examined as correlations of .90 or greater indicate possible problems with multicollinearity (Tabachnick \& Fiddell, 2013). The variables SUB12 'drug use interferes with interpersonal relationships' and SUB13 'drug use interferes with physical or emotional wellbeing' were strongly correlated, $r(48)=.90, p<.001$. To explore if these variables were multicollinear, they were retained for further analysis. The remaining variables were within an acceptable range ( $r=-.00$ to .87$)$; correlation tables for the gender-informed predictors are provided in Appendix A-K. Collinearity diagnostics were examined to determine the presence of multicollinearity for the variables SUB12 and SUB13. A conditioning index with a value greater than 30 for a given dimension along with a variance proportion greater than .50 for at least two variables indicates multicollinearity. The last two roots (dimensions) of the SUB12 and SUB13 variables demonstrated values greater than 30 . The last root also demonstrated a variance proportion of .85 for the variable SUB12 and a variance proportion of 
.75 for the variable SUB13. The Variance Inflation Factor (VIF) was also examined, with a VIF greater than 10.00 indicating multicollinearity. None of the variables were greater than 10.00 , however, SUB12 had a VIF of 9.88 and SUB13 had a VIF of 8.51. As recommended by Tabachnick and Fiddell (2013), the variables SUB12 and SUB13 were retained as the only goal of analysis is prediction.

\section{Descriptive Statistics}

Descriptive statistics for the number and percentage of women offenders who committed violent or non-violent index offences by the total sample, Indigenous women, and nonIndigenous women are presented in Table 2. Chi-square tests of independence were conducted for comparisons between Indigenous and non-Indigenous women offenders. In comparison to the non-Indigenous women offenders' base rate of violent index offences (48\%), the base rate is approximately 21 percentage points higher for Indigenous women offenders (69\%). A chi-square test of independence revealed significant differences for Indigenous and non-Indigenous women offenders, $\chi^{2}(1, N=1548)=65.72, p<.001, \Phi=.21$. See Table 2 for more details. Table 2

Number and Percentage of Women Offenders that Committed Violent or Non-Violent Index Offences for the Total Sample, Indigenous Offenders and non-Indigenous Offenders

\begin{tabular}{lcccccc}
\hline \multirow{2}{*}{ Offence Type } & Total Sample & $\begin{array}{c}\text { Indigenous } \\
\text { Women }\end{array}$ & $\begin{array}{c}\text { Non-Indigenous } \\
\text { Women }\end{array}$ & $\chi^{2}$ & $\Phi_{\mathrm{c}}$ \\
\cline { 2 - 5 } & $\%(n / 1555)$ & $\%(n / 577)$ & $\%(n / 971)$ & & \\
\hline Violent Offences & $\mathbf{5 6 . 1 ( 8 7 2 )}$ & $\mathbf{6 9 . 2 ( 3 9 9 )}$ & $\mathbf{4 8 . 0}(\mathbf{4 6 6})$ & $\mathbf{6 5 . 7 2 * * *}$ & $\mathbf{. 2 1}$ \\
Homicide & $13.2(205)$ & $17.7(102)$ & $10.5(102)$ & $16.28 * * *$ & .10 \\
Robbery & $19.5(304)$ & $23.9(138)$ & $17.0(165)$ & & $11.02 * *$ & .08 \\
Assault & $14.7(229)$ & $21.5(124)$ & $10.3(100)$ & & $36.63 * * *$ & .16 \\
& & & & & (Continued)
\end{tabular}




\begin{tabular}{lccccc}
\hline \multirow{2}{*}{ Offence Type } & Total Sample & $\begin{array}{c}\text { Indigenous } \\
\text { Women }\end{array}$ & $\begin{array}{c}\text { Non-Indigenous } \\
\text { Women }\end{array}$ & \multirow{2}{*}{$\chi^{2}$} & $\Phi_{\mathrm{c}}$ \\
\cline { 2 - 5 } & $\%(n / 1555)$ & $\%(n / 577)$ & $\%(n / 971)$ & & \\
\hline Sexual $^{\mathrm{a}}$ & $3.6(56)$ & $2.1(12)$ & $4.5(44)$ & 6.24 & .07 \\
Other $^{\mathrm{a}}$ & $5.0(78)$ & $4.0(23)$ & $5.7(55)$ & 2.13 & .04 \\
Non-violent Offences & $\mathbf{4 3 . 9 ( 6 8 3 )}$ & $\mathbf{3 0 . 8 ( 1 7 8 )}$ & $\mathbf{5 2 . 0 ( 5 0 5 )}$ & $\mathbf{6 5 . 7 2 * * *}$ &. $\mathbf{. 2 1}$ \\
Drug & $21.8(339)$ & $13.0(75)$ & $27.2(264)$ & $42.61 * * *$ & .17 \\
Property & $11.6(181)$ & $8.3(48)$ & $13.7(133)$ & $10.14 * *$ & .08 \\
Other & $10.5(163)$ & $9.5(55)$ & $11.1(108)$ & .97 & .03 \\
\hline
\end{tabular}

Note. $\Phi_{\mathrm{c}}=$ Cramer's V. ${ }^{\mathrm{a}}$ Sexual a ${ }^{\mathrm{a}} \mathrm{Other}=$ The results for Sexual and Other categories should be interpreted cautiously given that 2 cells have an expected count less than 5 .

Due to the number of comparisons to assess offence type, a Bonferonni adjustment was made ( $p$ $=.05 / 10=.005)$.

$* * p<.005 ; * * * p<.001$

On average, the total sample of women offenders were incarcerated for 795.50 days ( $S D$ $=442.12$ ) during the study timeframe. As Table 3 illustrates, there were significant differences between the Indigenous offenders and non-Indigenous offenders in terms of reintegration level, accountability level, and motivation level. Specifically, the majority of both Indigenous and nonIndigenous offenders had a medium Reintegration level and Accountability level. The majority of Indigenous and non-Indigenous offenders also had a medium and high motivation levels, respectively. However, Indigenous offenders had lower Reintegration levels than non-Indigenous offenders. Non-Indigenous offenders had a higher Accountability and Motivation level than Indigenous offenders. Chi-square analysis revealed the differences in Reintegration, Accountability and Motivation levels for Indigenous and non-Indigenous women were statistically significant. Details are provided in Table 3 below. 
Table 3

Reintegration Level, Accountability Level, and Motivation Level for the Total Sample, Indigenous Offenders, and non-Indigenous Offenders

$\begin{array}{ccc}\text { Total Sample } & \begin{array}{c}\text { Indigenous } \\ \text { Offenders }\end{array} & \begin{array}{c}\text { Non-Indigenous } \\ \text { Offenders }\end{array}\end{array} \chi^{2} \quad \Phi_{\mathrm{c}}$

\section{Reintegration Level}

\begin{tabular}{|c|c|c|c|c|}
\hline Low & $23.3(363)$ & $30.2(174)$ & $19.4(188)$ & $67.93 * * *$ \\
\hline Medium & $59.8(930)$ & $62.4(360)$ & $58.1(564)$ & \\
\hline High & $16.8(262)$ & $7.5(43)$ & $22.6(219)$ & \\
\hline
\end{tabular}

Accountability Level ${ }^{a}$

$\begin{array}{lccccc}\text { Low } & 10.8(168) & 8.3(48) & 12.3(119) & 23.13^{* * *} & .12 \\ \text { Medium } & 57.7(897) & 65.3(377) & 53.0(515) & & \\ \text { High } & 31.4(489) & 26.3(152) & 34.6(336) & & \\ \text { Motivation Level } & & & & & \\ \text { Low } & 4.8(75) & 3.5(20) & 5.6(54) & 11.85^{* *} & .09 \\ \text { Medium } & 48.6(756) & 53.9(311) & 45.4(441) & & \\ \text { High } & 46.6(724) & 42.6(246) & 49.0(476) & & \end{array}$

Note. ${ }^{a}$ Accountability Level $n=1$ missing for overall sample and non-Indigenous sample.

The results for Accountability Level should be interpreted cautiously given that 2 cells have an expected count less than 5 . Due to the number of comparisons to for reintegration, accountability and motivation levels, a Bonferonni adjustment was made $(p=.05 / 3=.017)$.

$* * p<.017 ; * * * p<.001$

Table 4 presents the security level of the total sample of women offenders, Indigenous offenders, and non-Indigenous offenders. Chi-square tests of independence were conducted for comparisons between Indigenous and non-Indigenous women offenders. In the total sample, the majority of women offenders had been designated to medium security level. However, more Indigenous women were designated to medium and maximum security levels while more nonIndigenous women were designated to minimum security. Chi-square analysis revealed that these 
differences in security designations for Indigenous and non-Indigenous women were statistically significant. It should also be noted that $6.0 \%(N=94)$ of the total sample were missing a final security level designation, thus the percentages do not add up to $100.0 \%$ in the analysis. Details are provided in Table 4 below.

Table 4

Final Security Level Designations for the Total Sample, Indigenous Offenders, and nonIndigenous Offenders

\begin{tabular}{lccccc}
\hline \multirow{2}{*}{$\begin{array}{l}\text { Security } \\
\text { Level }\end{array}$} & Total Sample & $\begin{array}{c}\text { Indigenous } \\
\text { Women }\end{array}$ & $\begin{array}{c}\text { Non-Indigenous } \\
\text { Women }\end{array}$ & & \\
\cline { 2 - 4 } & $\%(\mathrm{n} / 1555)$ & $\%(\mathrm{n} / 547)$ & $\%(\mathrm{n} / 908)$ & & $\chi^{2}$ \\
\hline Minimum & $28.7(446)$ & $16.8(97)$ & $35.9(349)$ & $68.83 * * *$ & .22 \\
Medium & $56.8(884)$ & $66.6(384)$ & $50.9(494)$ & $35.59 * * *$ & .16 \\
Maximum & $8.4(131)$ & $11.4(66)$ & $6.7(65)$ & $10.03^{* * *}$ & .08 \\
\hline
\end{tabular}

Note. $\Phi_{\mathrm{c}}=$ Cramer's V.

These results should be interpreted cautiously given that 2 cells have an expected count less than 5 . Due to the number of comparisons for each security level, a Bonferonni adjustment was made $(p=.05 / 3=.017)$.

$* * p<.017 ; * * * p<.001$

Table 5 provides descriptive statistics for the Institutional Adjustment subscale and Security Risk Score of the CRS for the total sample (including the mean, standard deviation, and range for the total sample). It should also be noted that descriptive statistics of the genderinformed items for Indigenous and non-Indigenous offenders (including the mean, standard deviation, and range for the total sample) are provided in Table 5. Further, t-tests were also conducted to assess the mean differences between Indigenous and non-Indigenous offenders on each of the gender-informed items. 
Table 5

Descriptive Statistics of the Institutional Adjustment Subscale and Security Risk Score for the Total Sample $N=1528$

\begin{tabular}{lccc}
\hline \multicolumn{1}{c}{ Measure } & $\mathrm{M}(S D)$ & Median & Range \\
\hline Institutional Adjustment & $41.09(27.51)$ & 38.00 & $0-158$ \\
Security Risk Score & $70.33(26.80)$ & 67.50 & $17-194$ \\
\hline
\end{tabular}

Note. $M=$ Mean. $S D=$ Standard Deviation.

As Table 6 indicates, Indigenous offenders scored significantly higher on the Institutional Adjustment scale of the CRS $(t(1006)=-10.47, p<.001$, Cohen's $d=0.58)$, indicating that Indigenous offenders receive greater scores in regards to the Institutional Adjustment scale than their non-Indigenous counterparts. Indigenous offenders scored significantly higher on the Security Risk Score of the CRS $(t(1426)=-6.33, p<.001$, Cohen's $d=0.34)$, indicating that Indigenous offenders receive greater Security Risk scores than their non-Indigenous counterparts.

Table 6

Descriptive Statistics of the Institutional Adjustment Subscale and Security Risk Score by Indigenous Status

\begin{tabular}{lccccccc}
\hline \multirow{2}{*}{ Measure } & \multicolumn{3}{c}{ Indigenous Offenders } & \multicolumn{2}{c}{ Non-Indigenous Offenders } \\
& \multicolumn{3}{c}{$(N=542)$} & & $(N=886)$ & $t$ \\
\cline { 2 - 6 } & $M(S D)$ & Median & Range & $M(S D)$ & Median & Range & \\
\hline Institutional & 50.81 & 42.00 & $0-140$ & 35.09 & 33.50 & $0-158$ & $-10.47^{* * *}$ \\
Adjustment & $(29.08)$ & & & $(24.76)$ & & & \\
Security & 75.95 & 74.00 & $17-194$ & 66.81 & 64.00 & $17-174$ & $-6.33^{* * *}$ \\
Risk Score & $(27.00)$ & & & $(26.14)$ & & & \\
\hline
\end{tabular}

Note. $M=$ Mean. $S D=$ Standard Deviation. $N=$ Number of participants in sample. $t=\mathrm{t}$-test score.

Table 7 provides the CRS security level for the total sample of women offenders, Indigenous offenders, and non-Indigenous offenders. Chi-square tests of independence were 
conducted for comparisons between Indigenous and non-Indigenous women offenders. In the total sample, the majority of women offenders had been designated by the CRS to medium security level. The majority of Indigenous women were also designated to medium security level. However, more Indigenous women were designated to medium and maximum security levels while more non-Indigenous women were designated to minimum security. Chi-square analysis revealed that these differences in security designations determined by the CRS for Indigenous and non-Indigenous women were statistically significant. It should also be noted that $6.0 \%(N=94)$ were missing a CRS security level designation, thus the percentages do not add up to $100.0 \%$. Details are provided in Table 7 below.

Table 7

CRS Security Level Designations for the Total Sample, Indigenous Offenders, and nonIndigenous Offenders

\begin{tabular}{lccccc}
\hline $\begin{array}{l}\text { Custody } \\
\text { Rating Scale }\end{array}$ & $\begin{array}{c}\text { Total Sample } \\
\%(n / 1528)\end{array}$ & $\begin{array}{c}\text { Indigenous } \\
\text { Women } \\
\%(n / 572)\end{array}$ & $\begin{array}{c}\text { Non-Indigenous } \\
\text { Women } \\
\%(n / 949)\end{array}$ & $\chi^{2}$ & $\Phi_{\mathrm{c}}$ \\
\hline Minimum & $38.0(581)$ & $27.9(151)$ & $48.4(429)$ & $60.41^{* * *}$ & .21 \\
Medium & $46.9(717)$ & $57.9(314)$ & $44.9(398)$ & $25.46^{* * *}$ & .13 \\
Maximum & $8.9(136)$ & $14.2(77)$ & $6.7(59)$ & $22.95^{* * *}$ & .13 \\
\hline
\end{tabular}

Note. $\Phi_{\mathrm{c}}=$ Cramer's V.

These results should be interpreted cautiously given that 2 cells have an expected count less than 5. Due to the number of comparisons for each security level, a Bonferonni adjustment was made $(p=.05 / 3=.017)$.

$* * p<.017 ; * * * p<.001$

Table 8 provides descriptive statistics for minor, major, and any misconduct charges for the total sample, Indigenous and non-Indigenous offenders (including the mean, standard deviation, and range for the total sample). Table 8 also provides t-tests, which were conducted to assess the mean differences between Indigenous and non-Indigenous offenders for institutional 
misconduct type. Table 9 provides the total number of minor, major and any misconduct charges for the total sample, Indigenous offenders and non-Indigenous offenders. For these tables, any misconduct includes an offender that received a minor or major charge. If an offender received a minor and major charge while incarcerated for the duration of the study, the charge that occurred first was used for the analysis while the charge that occurred afterwards was excluded.

Table 8

Mean Number of Misconducts for Total Sample, Indigenous Offenders, and non-Indigenous Offenders

\begin{tabular}{lccccccc}
\hline & \multicolumn{2}{c}{$\begin{array}{c}\text { Total Sample } \\
\text { Misconduct } \\
\text { Type }\end{array}$} & \multicolumn{2}{c}{$\begin{array}{c}\text { Indigenous Offenders } \\
(N=1528)\end{array}$} & $\begin{array}{c}\text { Non-Indigenous } \\
\text { Offenders } \\
(N=572)\end{array}$ & \multicolumn{2}{c}{$\begin{array}{c}(N=949) \\
\end{array}$} \\
\cline { 2 - 7 } & $M(S D)$ & Range & $M(S D)$ & Range & $M(S D)$ & Range \\
\hline Minor & $2.73(6.01)$ & $0-118$ & $3.90(7.95)$ & $0-118$ & $2.04(4.34)$ & $0-46$ & $-5.17 * * *$ \\
Major & $0.99(3.27)$ & $0-85$ & $1.03(2.41)$ & $0-31$ & $0.97(3.71)$ & $0-85$ & -.34 \\
Any & $3.72(8.04)$ & $0-149$ & $4.93(9.63)$ & $0-149$ & $3.01(6.87)$ & $0-93$ & $-4.18^{* * *}$ \\
\hline
\end{tabular}

Note. $M=$ Mean. $S D=$ Standard Deviation. $N=$ Number of participants in sample. $t=\mathrm{t}$-test score.

Table 9

Type of Misconducts by Misconduct Type for Total Sample, Indigenous Offenders and nonIndigenous Offenders

\begin{tabular}{lccc}
\hline \multirow{2}{*}{$\begin{array}{c}\text { Misconduct } \\
\text { Type }\end{array}$} & Total Sample & Indigenous Offenders & $\begin{array}{c}\text { Non-Indigenous } \\
\text { Offenders }\end{array}$ \\
\cline { 2 - 4 } & $\%(n / 1528)$ & $\%(n / 572)$ & $\%(n / 949)$ \\
\hline Minor & $53.1(812)$ & $63.1(361)$ & $47.3(449)$ \\
Major & $30.5(466)$ & $36.0(206)$ & $27.4(260)$ \\
Any & $58.8(899)$ & $68.4(391)$ & $53.3(506)$ \\
\hline
\end{tabular}

Note. $\Phi_{\mathrm{c}}=$ Cramer's V.

Due to the number of comparisons to assess offence type, a Bonferonni adjustment was made $(p$ $=.05 / 2=.005)$.

$* * p<.025 ; * * * p<.001$ 
As Table 9 indicates, the majority of women offenders in the total sample had minor and any misconduct charges. Indigenous women offenders and non-Indigenous women offenders also had more minor and any misconduct charges. However, Indigenous women offenders had greater base rates of minor, major and any misconduct charges than non-Indigenous women offenders. The t-tests (provided in Table 8) found that Indigenous offenders scored significantly higher on minor misconducts $(t(779)=-5.17, p<.001$, Cohen's $d=0.29)$, and any misconducts $(t(923)=-4.18, p<.001$, Cohen's $d=0.23)$. These results indicate that Indigenous offenders engage in more minor and any institutional misconducts than their non-Indigenous counterparts. It should be noted the time at risk for Indigenous offenders was approximately 7 years, while the time at risk for non-Indigenous offenders was approximately 10 years. A t-test for the time at risk was conducted for both Indigenous and non-Indigenous offenders, and results revealed no significant differences in time at risk $(t(1519)=-1.06, p=.29$, Cohen's $d=0.06)$.

\section{Research Question 1: Which gender-informed variables predict institutional misconduct?}

A series of univariate survival analyses using each gender informed variable to predict time to any misconduct was conducted to explore research question 1. Survival analysis is used to analyze time to event data, and describes the length of time from an origin in time to the event of interest (Kartsonaki, 2016). The range of the time at risk for the analysis was between October 18, 2006 and January 8, $2017(M=579.18$ days, $S D=402.53$ days). Further, it should be noted the range of dates for the time at risk for Indigenous offenders was between June 23, 2009 to January 8, 2017 ( $M=594.37$ days, $S D=396.76$ days $)$; while the range of dates for the time at risk for non-Indigenous offenders was between October 18, 2006 to January 8, 2017 ( $M=571.84$ days, $S D=406.82$ days). 
Tables 10 to 15 (as will be described below) present the survival analyses for all the gender-informed domains (Mental Health, Substance Abuse, Parental/Family Issues, Relationship Dysfunction, Victimization and Personal/Emotional). These tables present the regression coefficients $(\beta)$, standard errors, Wald's statistic, Hazard ratio, and confidence intervals. As presented in Table 10, survival analysis was conducted on the gender-informed domain Mental Health for any misconducts. Each measure from the gender-informed Mental Health variable was used to predict likelihood of any misconducts. Results indicate that Historical Suicide (previous suicide/self-harm behaviour and serious thoughts of suicide) significantly predicted any misconducts, with more severe previous suicide/self-harm behavior and serious thoughts of suicide predicting greater likelihood of engaging in any misconducts. Historical Suicide had a positive effect on engaging in any misconducts. None of the remaining Mental Health items predicted any misconducts. Details are presented in Table 10 below.

Table 10

Survival Analysis of Any Misconduct and the Gender-informed Variable Mental Health with the Total Sample

\begin{tabular}{lccccc}
\hline & $\beta$ & $S E$ & $\begin{array}{c}\chi^{2} \text { (Wald's } \\
\text { Statistic) }\end{array}$ & $\begin{array}{c}\text { Hazard } \\
\text { Ratio }\end{array}$ & $95 \%$ CI \\
\hline Severity of Distress $^{\mathrm{a}}$ & .13 & $(.09)$ & 2.16 & 1.13 & {$[.96,1.34]$} \\
DHS Depression & .02 & $(.02)$ & 1.12 & 1.02 & {$[.99,1.05]$} \\
DHS Current Suicidal Ideation & -.07 & $(.13)$ & .30 & .93 & {$[.72,1.20]$} \\
DHS Hopelessness & .00 & $(.02)$ & .01 & 1.00 & {$[.96,1.05]$} \\
DHS Cognitive Suicide & -.03 & $(.11)$ & .09 & .97 & {$[.78,1.20]$} \\
DHS Historical Suicide & .13 & $(.03)$ & $29.04 * * *$ & 1.14 & {$[1.09,1.20]$} \\
\hline
\end{tabular}

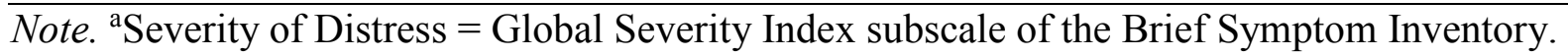
$* p<.05 ; * * p<.01 ; * * * p<.001$ 
As presented in Table 11, survival analysis was also conducted on the gender-informed domain Substance Abuse for any misconducts. Each item from the gender-informed Substance Abuse domain was used individually to predict likelihood of any misconducts. Results indicate the items 'has gone on drug-taking bouts or binges' and 'becomes violent when drinking or using drugs' significantly predicted any misconducts. Endorsement of these two variables predicted a greater likelihood of any misconducts. No other items from the gender-informed Substance Abuse variable were significant for any misconducts. Details are presented in Table 11 below. Table 11

Survival Analysis of Any Misconduct and the Gender-informed Variable Substance Abuse with the Total Sample

\begin{tabular}{|c|c|c|c|c|c|}
\hline & $\beta$ & $S E$ & $\begin{array}{l}\chi^{2}(\text { Wald's } \\
\text { Statistic })\end{array}$ & $\begin{array}{l}\text { Hazard } \\
\text { Ratio }\end{array}$ & $95 \% \mathrm{CI}$ \\
\hline Early age alcohol use & -.09 & $(.12)$ & .54 & .92 & {$[.73,1.16]$} \\
\hline $\begin{array}{l}\text { Frequently engages in binge } \\
\text { drinking }\end{array}$ & -.05 & $(.14)$ & .13 & .95 & {$[.72,1.26]$} \\
\hline $\begin{array}{l}\text { Has combined the use of alcohol } \\
\text { and drugs }\end{array}$ & -.06 & $(.12)$ & .27 & .94 & {$[.75,1.19]$} \\
\hline $\begin{array}{l}\text { Alcohol use interferes with } \\
\text { employment }\end{array}$ & -.11 & $(.14)$ & .65 & .89 & {$[.68,1.17]$} \\
\hline $\begin{array}{l}\text { Alcohol use interferes with } \\
\text { interpersonal relationships }\end{array}$ & .10 & $(.19)$ & .28 & 1.10 & {$[.77,1.59]$} \\
\hline $\begin{array}{l}\text { Alcohol use interferes with physical } \\
\text { or emotional wellbeing }\end{array}$ & -.10 & $(.18)$ & .34 & .90 & {$[.64,1.28]$} \\
\hline $\begin{array}{l}\text { Excessive alcohol use is part of the } \\
\text { offender's lifestyle }\end{array}$ & .04 & $(.18)$ & .05 & 1.04 & {$[.74,1.47]$} \\
\hline Early age drug use & .19 & $(.12)$ & 2.51 & 1.21 & {$[.96,1.54]$} \\
\hline $\begin{array}{l}\text { Has gone on drug-taking bouts or } \\
\text { binges }\end{array}$ & .35 & $(.16)$ & $4.74 *$ & 1.42 & {$[1.04,1.95]$} \\
\hline
\end{tabular}




\begin{tabular}{|c|c|c|c|c|c|}
\hline & $\beta$ & $S E$ & $\begin{array}{l}\chi^{2} \text { (Wald's } \\
\text { Statistic) }\end{array}$ & $\begin{array}{l}\text { Hazard } \\
\text { Ratio }\end{array}$ & $95 \% \mathrm{CI}$ \\
\hline $\begin{array}{l}\text { Has combined the use of different } \\
\text { drugs }\end{array}$ & .16 & $(.14)$ & 1.37 & 1.17 & {$[.90,1.54]$} \\
\hline $\begin{array}{l}\text { Drug use interferes with } \\
\text { employment }\end{array}$ & -.13 & $(.14)$ & .87 & .88 & {$[.66,1.16]$} \\
\hline $\begin{array}{l}\text { Drug use interferes with } \\
\text { interpersonal relationships }\end{array}$ & .28 & $(.24)$ & 1.35 & 1.33 & {$[.82,2.14]$} \\
\hline $\begin{array}{l}\text { Drug use interferes with physical or } \\
\text { emotional wellbeing }\end{array}$ & .12 & (.24) & .26 & 1.13 & {$[.71,1.80]$} \\
\hline $\begin{array}{l}\text { Regular drug use is part of the } \\
\text { offender's lifestyle }\end{array}$ & .05 & $(.18)$ & .07 & 1.05 & {$[.73,1.51]$} \\
\hline $\begin{array}{l}\text { Alcohol or drug use has resulted in } \\
\text { law violations }\end{array}$ & .11 & (.18) & .34 & 1.11 & {$[.78,1.59]$} \\
\hline $\begin{array}{l}\text { Becomes violent when drinking or } \\
\text { using drugs }\end{array}$ & .51 & $(.10)$ & $25.74 * * *$ & 1.67 & {$[1.37,2.03]$} \\
\hline $\begin{array}{l}\text { Alcohol and/or drug use is part of } \\
\text { the offence cycle }\end{array}$ & -.12 & $(.20)$ & .35 & .89 & {$[.61,1.31]$} \\
\hline Previously referred to programs & .04 & $(.09)$ & .20 & 1.04 & {$[.88,1.24]$} \\
\hline LOG Alcohol Dependence Scale ${ }^{a}$ & .18 & $(.24)$ & .58 & 1.20 & {$[.75,1.93]$} \\
\hline Drug Abuse Screening Test & .07 & $(.05)$ & 2.09 & 1.07 & {$[.98,1.17]$} \\
\hline
\end{tabular}

${ }^{*} p<.05 ; * * p<.01 ; * * * p<.001$

As presented in Table 12, survival analysis was conducted on each measure from the gender-informed Relationship Dysfunction domain and each item was used individually to predict likelihood of any misconducts. Results indicate the items 'inability to maintain enduring intimate relationship,' 'intimate relationships have been problematic,' and 'perpetuated spousal violence' were significant predictors of any misconducts. These items had a positive effect on engaging in any misconducts; as more severe problems maintaining an enduring relationship, relationship problems and perpetuating spousal violence predicted greater likelihood of any 
misconducts. None of the remaining Relationship Dysfunction items were related to any misconducts. Details are provided in Table 12 below.

Table 12

Survival Analysis of Any Misconduct with the Gender-informed Variable Relationship Dysfunction with the Total Sample

\begin{tabular}{lccccc}
\hline & $\beta$ & $S E$ & $\begin{array}{c}\chi^{2} \text { (Wald's } \\
\text { Statistic) }\end{array}$ & $\begin{array}{c}\text { Hazard } \\
\text { Ratio }\end{array}$ & $95 \%$ CI \\
\hline $\begin{array}{l}\text { Inability to maintain enduring intimate } \\
\text { relationship }\end{array}$ & .32 & $(.08)$ & $17.57 * * *$ & 1.38 & {$[1.19,1.60]$} \\
$\begin{array}{l}\text { Intimate relationships have been } \\
\text { problematic }\end{array}$ & .29 & $(.12)$ & $5.93 *$ & 1.33 & {$[1.06,1.68]$} \\
$\begin{array}{l}\text { Perpetuated spousal violence } \\
\text { Marital status }\end{array}$ & .34 & $(.08)$ & $19.92 * * *$ & 1.41 & {$[1.21,1.64]$} \\
\hline$p<.05 ; * * p<.01 ; * * * p<.001$ & .06 & $(.08)$ & .65 & 1.07 & {$[.91,1.25]$} \\
\hline
\end{tabular}

As presented in Table 13, survival analysis was conducted on each item from the genderinformed Parental/Family Issues domain for any misconducts. Results indicate the items 'family member criminally active during childhood,' 'relationships with parental figure were negative during childhood' and 'prosocial support from friends is limited' significantly predicted any misconducts. Having a family member that was criminally active during childhood, having negative parental relationships during childhood and having limited prosocial support from friends predicted greater likelihood for having any institutional misconduct charges. None of the remaining Parental/Family Issues items predicted any institutional misconducts. Details are provided in Table 13 below. 
Table 13

Survival Analysis of Any Misconduct and Parental/Family Issues with the Total Sample

\begin{tabular}{|c|c|c|c|c|c|}
\hline & $\beta$ & $S E$ & $\begin{array}{l}\chi^{2}(\text { Wald's } \\
\text { Statistic })\end{array}$ & $\begin{array}{l}\text { Hazard } \\
\text { Ratio }\end{array}$ & $95 \% \mathrm{CI}$ \\
\hline $\begin{array}{l}\text { Relationships with parental figure were } \\
\text { negative during childhood }\end{array}$ & .18 & $(.09)$ & $4.18^{*}$ & 1.20 & {$[1.01,1.43]$} \\
\hline $\begin{array}{l}\text { Family members criminally active } \\
\text { during childhood }\end{array}$ & .35 & $(.08)$ & $17.93 * * *$ & 1.42 & {$[1.21,1.67]$} \\
\hline $\begin{array}{l}\text { Has significant difficulties handling } \\
\text { parenting responsibilities }\end{array}$ & -.00 & $(.08)$ & .00 & 1.00 & {$[.86,1.16]$} \\
\hline Prosocial family support is limited & .09 & $(.08)$ & 1.08 & 1.09 & {$[.93,1.28]$} \\
\hline $\begin{array}{l}\text { Prosocial support from friends is } \\
\text { limited }\end{array}$ & .61 & $(.10)$ & $35.61 * * *$ & 1.84 & {$[1.51,2.25]$} \\
\hline
\end{tabular}

$* p<.05 ; * * p<.01 ; * * * p<.001$

As presented in Table 14, survival analysis was also conducted on the each of the items for the gender-informed domain Victimization for any misconducts. Results indicate that the item 'limited attachment to family unit during childhood' and 'abused during childhood' was a significant predictor of any misconducts. Having limited attachment to the family unit during childhood and being abused during childhood predicted greater likelihood of and shorter time to any misconducts. None of the remaining Parental/Family Issues items were related to any misconducts. Details are provided in Table 14 below.

Table 14

Survival Analysis of Any Misconduct with the Gender-informed Variable Victimization with the Total Sample

\begin{tabular}{llllll}
\hline & $\beta$ & $S E$ & $\begin{array}{c}\chi^{2} \text { (Wald's } \\
\text { Statistic) }\end{array}$ & $\begin{array}{c}\text { Hazard } \\
\text { Ratio }\end{array}$ & $95 \%$ CI \\
\hline $\begin{array}{l}\text { Limited attachment to family unit } \\
\text { during childhood }\end{array}$ & .34 & $(.08)$ & $18.79 * * *$ & 1.40 & {$[1.20,1.64]$} \\
& & & & &
\end{tabular}

(Continued) 


\begin{tabular}{lccccc}
\hline & $\beta$ & $S E$ & $\begin{array}{c}\chi^{2} \text { (Wald's } \\
\text { Statistic) }\end{array}$ & $\begin{array}{c}\text { Hazard } \\
\text { Ratio }\end{array}$ & 95\% CI \\
\hline Abused during childhood & .20 & $(.09)$ & $4.79^{*}$ & 1.22 & {$[1.02,1.46]$} \\
$\begin{array}{l}\text { Witnessed family violence during } \\
\text { childhood }\end{array}$ & .15 & $(.09)$ & 3.23 & 1.17 & {$[.99,1.38]$} \\
\begin{tabular}{l} 
Victimized by spousal abuse \\
\hline
\end{tabular} & .11 & $(.08)$ & 1.90 & 1.12 & {$[.95,1.31]$} \\
\hline
\end{tabular}

${ }^{*} p<.05 ; * * p<.01 ; * * * p<.001$

As presented in Table 15, survival analysis was conducted for each item on the genderinformed domain Personal/Emotional for any misconducts. Results indicate the items 'assertiveness skills are limited,' 'frequently feels intense anger,' 'frequently suppresses anger,' 'has difficulty solving interpersonal problems,' 'gives up easily when challenged,' and 'has low frustration tolerance' significantly predicted any misconducts. Frequently feeling intense anger, frequently suppressing anger, having difficulties solving interpersonal problems, giving up easily when challenged and having low frustration tolerance had a positive effect on any misconducts as these items predicted greater likelihood of any misconducts. However, limited assertiveness skills had a negative effect on engaging in any misconducts, as this item predicted a lesser likelihood of any misconducts. None of the remaining Personal/Emotional items were related to any misconducts. Details are provided in Table 15 below.

Table 15

Survival Analysis of Any Misconduct with the Gender-informed Personal/Emotional Variable with the Total Sample

\begin{tabular}{llllll}
\hline & $\beta$ & $S E$ & $\begin{array}{c}\chi^{2} \text { (Wald's } \\
\text { Statistic) }\end{array}$ & $\begin{array}{c}\text { Hazard } \\
\text { Ratio }\end{array}$ & $95 \% \mathrm{CI}$ \\
\hline Has difficulty coping with stress & .04 & $(.11)$ & .14 & 1.04 & $\begin{array}{c}{[.84,1.30]} \\
\text { (Continued) }\end{array}$
\end{tabular}




\begin{tabular}{lccccc}
\hline & $\beta$ & $S E$ & $\begin{array}{c}\chi^{2} \text { (Wald's } \\
\text { Statistic) }\end{array}$ & $\begin{array}{c}\text { Hazard } \\
\text { Ratio }\end{array}$ & $95 \%$ CI \\
\hline $\begin{array}{l}\text { Gives up easily when } \\
\text { challenged }\end{array}$ & .16 & $(.08)$ & $3.88^{*}$ & 1.18 & {$[1.00,1.38]$} \\
Assertiveness skills are limited & -.20 & $(.08)$ & $6.66^{*}$ & .82 & {$[.70, .95]$} \\
$\begin{array}{l}\text { Has difficulty solving } \\
\text { interpersonal problems }\end{array}$ & .20 & $(.09)$ & $4.42^{*}$ & 1.22 & {$[1.01,1.46]$} \\
$\begin{array}{l}\text { Frequently feels intense anger } \\
\text { Frequently supresses anger }\end{array}$ & .34 & $(.09)$ & $13.22^{* * *}$ & 1.40 & {$[1.17,1.68]$} \\
Has low frustration tolerance & .19 & $(.08)$ & $5.24 *$ & 1.21 & {$[1.03,1.43]$} \\
$*$ * $p<.05 ; * * p<.01 ; * * *<.001$ & & $(.09)$ & $26.25 * * *$ & 1.59 & {$[1.33,1.90]$} \\
\hline
\end{tabular}

\section{Creation of the Gender-Informed Scales}

Two scales were created from the items in each domain that were found to be predictive of institutional misconducts. Out of the original 60 items that were potential predictors of misconducts, a total of 17 items from each domain were found to be significant in predicting institutional misconducts. It should be noted the item, 'assertiveness skills are limited' had a negative beta value. This is contrary to previous findings in the literature, since predictors relating to the Personal/Emotional domains were found to be a predictor of engaging in institutional misconducts (e.g., Van Voorhis et al., 2010). As such, this item was dropped from further analysis due to lack of theoretical support for demonstrating a negative beta value. Hence, a total of 16 items were included in the creation of the gender-informed scales. The list of items that were included in the creation of the gender-informed scales are presented in Table 16 below. 
Table 16

Gender-informed Predictors of Institutional Misconducts Used to Create the Gender-informed Scales

Gender-Informed Variable
Mental Health
Substance Abuse
Relationship Dysfunction

Parental/Family Issues

Personal/Emotional

Victimization

\section{Total Sample}

\section{DHS Historical Suicide}

Becomes violent when drinking or using drugs

Has gone on drug-taking bouts or binges

Inability to maintain enduring intimate relationship

Perpetuated spousal violence

Intimate relationships have been problematic

Prosocial support from friends is limited

Relationships with parental figure were negative during childhood

Family members criminally active during childhood

Limited attachment to family unit during childhood

Abused during childhood

Has low frustration tolerance

Has difficulty solving interpersonal problems

Frequently feels intense anger

Frequently supresses anger

Gives up easily when challenged

It should be noted that all the predictors presented in Table 16 above were all dichotomous variables and were derived from the DFIA-R measure; with the exception of the DHS Historical Suicide item (this item was derived from CoMHISS information). Since the DHS Historical item was a continuous variable, the item was dichotomized to be included for further analysis. As such, the DHS Historical Suicide item from the Mental Health domain was 
dichotomized utilizing a median split (value of 0.00 indicates at the median or below, while a value of 1.00 indicates above the median).

Further, the DHS Historical Suicide item comprised of $27.7 \%$ of data, and such amount of missing data in this item may have impeded the validity of the findings. However, due to the theoretical importance of the item, the inclusion of the DHS Historical Suicide item was also imperative. As such, two scales were created: one scale including the DHS Historical Suicide item and one scale excluding the DHS Historical Suicide item. The first gender-informed scale that was created, the Gender-Informed Version 1 Continuous (GI-V1c) scale, included all 16 predictors listed in Table 16 above. Specifically, the GI-V1c included the dichotomized DHS Historical Suicide item and the remaining 15 predictors from the DFIA-R.

Next, the second gender-informed scale, the Gender-Informed Version 2 Continuous (GIV2c) scale, was created. The GI-V2c comprised of all 15 DFIA-R predictors displayed in Table 16 above, but excluded the dichotomized DHS Historical Suicide item. As such, GI-V1c ranged from 0.00 to $16.00(M=7.47, S D=3.90)$, while GI-V2c ranged from 0.00 to $15.00(M=7.17$, $S D=3.77)$. For Indigenous offenders, GI-V1c ranged from 0.00 to $16.00(M=9.24, S D=3.56)$, while GI-V2c ranged from 0.00 to $15.00(M=8.95, S D$ 3.44). For non-Indigenous offenders, GIV1c ranged from 0.00 to $16.00(M=6.40, S D=3.71)$, while GI-V2c ranged from 0.00 to 15.00 $(M=6.10, S D=3.56)$.

Since the GI-V1c and GI-V2c scales are continuous, it was determined that it would be advantageous to trichotomize the GI-V1c and GI-V2c scales. The CRS is restricted to three security level designations (minimum, medium, and maximum security) that can be used to predict likelihood of misconduct, thus it is advantageous for the GI-V1c and GI-V2c scales to demonstrate greater predictive accuracy than the CRS for institutional misconducts. 
Consequently, the gender-informed scales (GI-V1c and GI-V2c) were trichotomized to eliminate any statistical advantage these continuous scales may have over the CRS.

First, a frequency distribution of the Custody Rating Scale was computed. The analysis indicated $38.0 \%$ of the total sample $(N=1528)$ were designated as minimum security, $46.9 \%$ as medium security and $8.9 \%$ as maximum security. Next, the gender-informed scales were trichotomized to mirror, as proximally as possible, the CRS distribution of minimum, medium, and maximum security designations. Since the gender-informed scales are intended to be created to assist in determining the security designation of women offenders, the proper distribution of security designations must also be reflected.

To do this, the two gender-informed scales (GI-V1c and GI-V2c) were trichotomized into 'low' (value of 1), 'medium' (value of 2) and 'high' (value of 3) groups. For the GI-V1c scale, $40.0 \%(N=611)$ of the total sample had scores that ranged from 0.00 to 6.00 , and were subsequently designated as the 'low' group. Next, $50.0 \%(N=764)$ of the total sample that had scores ranging from 7.00 to 12.00 were designated as the 'medium' group, and $10.0 \%(N=153)$ of the total sample that had scores ranging from 13.00 to 16.00 were designated as the 'high' group. This new variable was renamed Gender-Informed Version 1 Trichotomized (GI-V1t). For the GI-V2c scale, $42.3 \%(N=647)$ of the total sample that had scores ranging from 0.00 to 6.00 were designated as the 'low' group, $48.7 \%(N=744)$ of the total sample that had scores ranging from 7.00 to 12.00 were designated as the 'medium' group, and $9.0 \%(N=137)$ of the total sample that had scores ranging from 13.00 to 15.00 were designated as the 'high' group. This new variable was renamed Gender-Informed Version 2 Trichotomized (GI-V2t). It should be noted that offenders that were designated to the 'high' group had the greatest genderinformed factors, while offenders designated to the 'low' group had the least gender-informed 
factors. All subsequent analysis will utilize both the continuous gender-informed scales (GI-V1c and GI-V2c) and trichtomized gender-informed scales (GI-V1t and GI-V2t).

Kaplan-Meier Analysis. To determine if the 'low,' 'medium,' and 'high' groups for the Gender-Informed Version 1 Trichotomized and Gender-Informed Version 2 Trichotomized scales appropriately distinguish between offenders that had the greatest time to failure from those that had the least time to failure, survival curves were produced using the Kaplain-Meier analysis. Further, survival curves from the Kaplain-Meier analysis was also produced for the Custody Rating Scale. The survival curves for the GI-V1t and GI-V2t scales and the Custody Rating Scale are presented in Figures 1-3 below.

As can be seen from Figures 1 and 2, the GI-V1t and GI-V2t scales distinguish offenders that had a greater time to failure from those that did not. Further, offenders in the 'high' group had a lesser time to failure and greater institutional charges in comparison to offenders in the 'low' and 'medium' groups. Further, offenders in the 'medium' group had a lesser time to failure and greater institutional charges in comparison to offenders in the 'low' group. Figure 3 reveals that offenders that had maximum security designations had the most failures (committing any misconduct) than offenders who had a medium or minimum security designation. Offenders that had a minimum security designation had the least failures than offenders who had a medium or maximum security designation. 


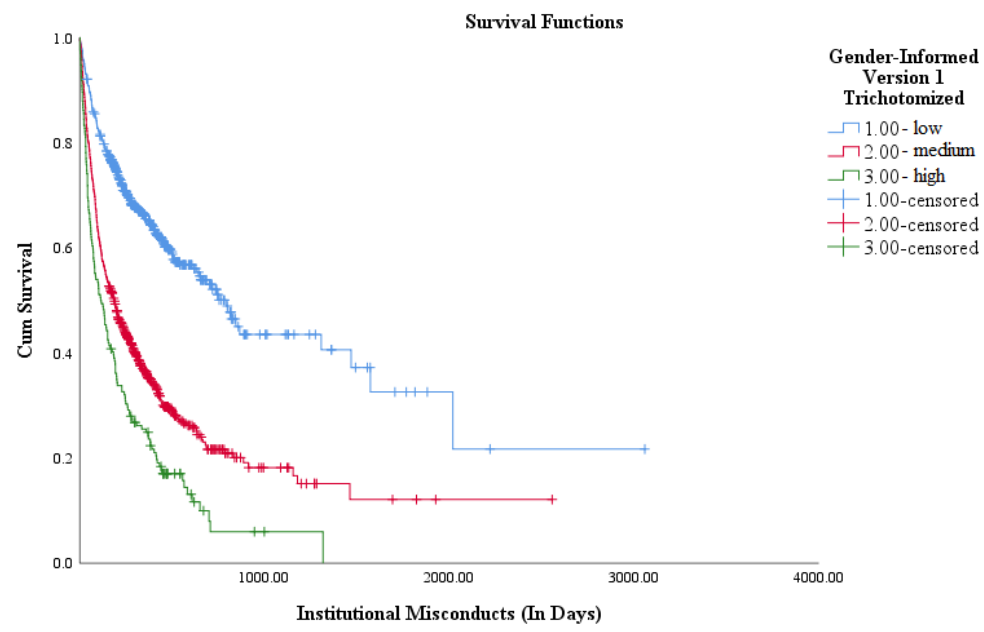

Figure 1. Survival curves for the Gender-Informed Version 1 Trichotomized scale for any misconducts with the total sample. The Gender-Informed Version 1 Continuous scale was trichotomized into 'low,' 'medium,' and 'high' groups. This scale was renamed the GenderInformed Version 1 Trichotomized scale. The value 'low' is presented numerically as ' 1 ,' the value 'medium' is presented numerically as '2,' and the value 'high' is presented numerically as '3.'

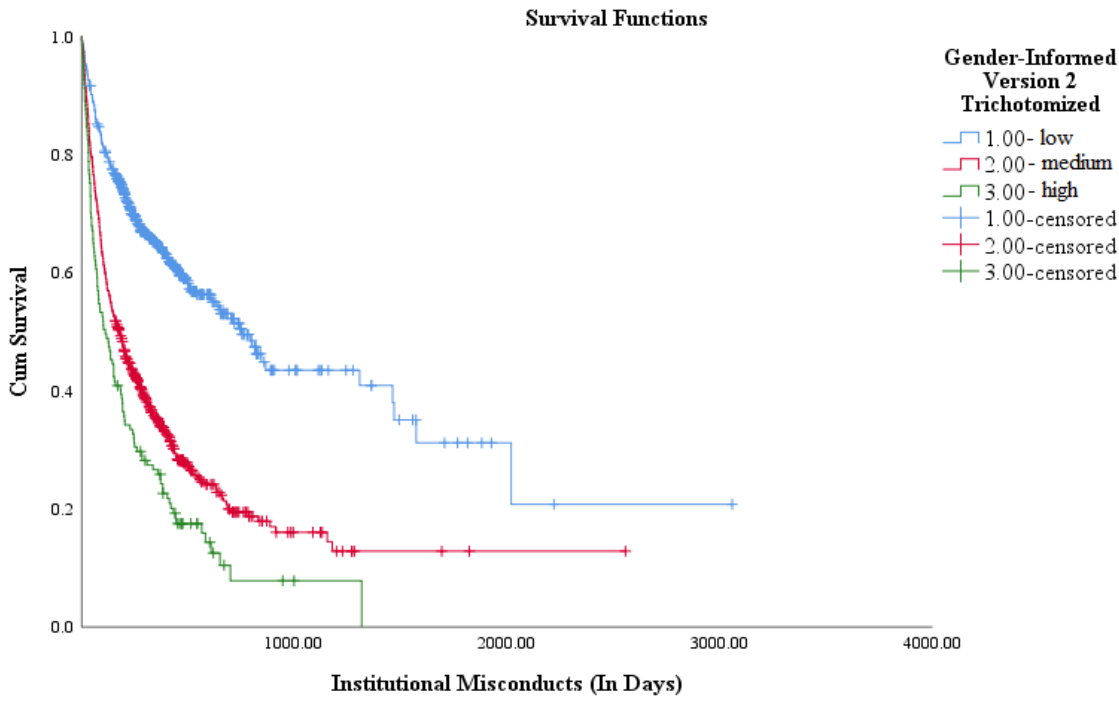

Figure 2. Survival curves for the Gender-Informed Version 2 Trichotomized scale for any misconduct with the total sample. The Gender-Informed Version 2 Continuous scale was tichotomized into 'low, 'medium,' and 'high' groups. This scale was renamed to the GenderInformed Version 2 Trichotomized scale. The value 'low' is presented numerically as ' 1 ,' the value 'medium' is presented numerically as ' 2 ,' and the value 'high' is presented numerically as '3.' 


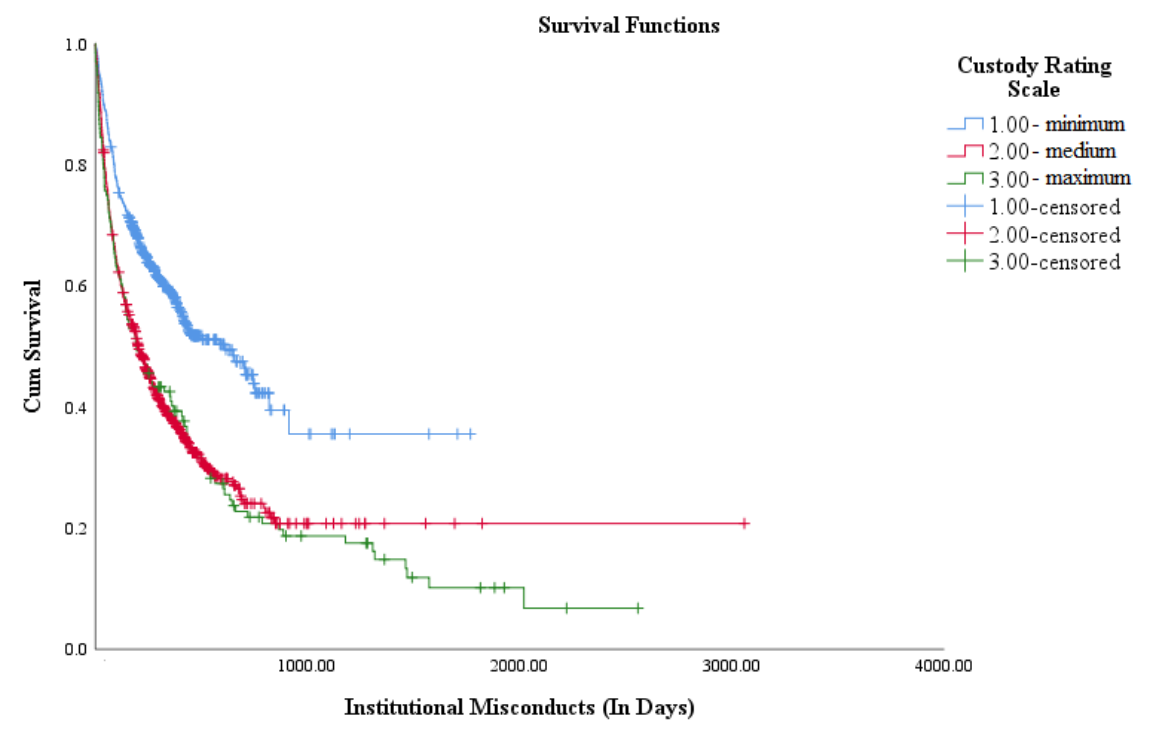

Figure 3. Survival curves for the CRS of any misconduct for the total sample. The value 1 indicates Minimum, 2 indicates Medium, and 3 indicates Maximum security designations.

Bivariate Correlations. Bivariate correlations were produced to explore the relationships between the GI-V1c, GI-V2c, GI-V1t and GI-V2t scales with institutional misconducts. Further, bivariate correlations were also created to determine the relationship between the Custody Rating Scale and institutional misconducts in this sample of women offenders. Bivariate correlations for each measure with any misconduct with the total sample, Indigenous offenders and nonIndigenous offenders are provided in Table 17 below. All measures demonstrated strong statistically significant correlations with any misconduct, as $p$ values were less than .001 . All of the gender-informed scales demonstrated stronger bivariate correlations than the Custody Rating Scale for any misconducts. Details are provided in Table 17 below 
Table 17

Bivariate Correlations with Institutional Misconduct for the Total Sample, Indigenous Offenders, and Non-Indigenous Offenders

\begin{tabular}{lccc}
\hline \multicolumn{1}{c}{ Measure } & $\begin{array}{c}\text { Total Sample } \\
(N=1528)\end{array}$ & $\begin{array}{c}\text { Indigenous } \\
\text { Offenders } \\
(N=572)\end{array}$ & $\begin{array}{c}\text { Non- } \\
\text { Indigenous } \\
\text { Offenders } \\
(N=949)\end{array}$ \\
\cline { 2 - 4 } & \multicolumn{2}{c}{ Institutional Misconduct } \\
\hline Custody Rating Scale & $.27 * * *$ & $.30 * * *$ & $.21 * * *$ \\
Gender-Informed Version 1 Continuous & $.39 * * *$ & $.34 * * *$ & $.38^{* * *}$ \\
Gender-Informed Version 2 Continuous & $.39 * * *$ & $.34 * * *$ & $.38 * * *$ \\
Gender-Informed Version 1 Trichotomized & $.35 * * *$ & $.30 * * *$ & $.34 * * *$ \\
Gender-Informed Version 2 Trichotomized & $.34 * * *$ & $.27 * * *$ & $.34 * * *$ \\
\hline
\end{tabular}

Note. Gender-Informed Version 1 Continuous is the gender-informed scale that contains all 16 gender-informed predictors of institutional misconducts. Gender-Informed Version 2 Continuous is the gender-informed scale that excludes the DHS Historical Suicide predictor but contains the remaining 15 gender-informed predictors of institutional misconducts. Gender-Informed Version 1 Trichotomized is the trichotomized Gender-Informed Version 1 Continuous scale. GenderInformed Version 2 Trichotomized is the trichotomized Gender-Informed Version 2 Continuous scale.

$* p<.05 ; * * p<.01 ; * * * p<.001$

Bivariate correlations were also produced for the relationship between the GI-V1c, GIV2c, GI-V1t and GI-V2t scales and the Custody Rating Scale. Table 18 provides bivariate correlations between the Custody Rating Scale and gender-informed scales for the total sample, Indigenous offenders, and non-Indigenous offenders. All the gender-informed scales demonstrated strong correlations with the Custody Rating Scale, as correlations reached statistical significance. However, it should be noted that for non-Indigenous offenders, correlations between the gender-informed scales and the CRS were weaker in comparison to Indigenous offenders and the total sample. Details are provided in Table 18 below. 
Table 18

Bivariate Correlations with the Custody Rating Scale for the Total Sample, Indigenous Offenders, and Non-Indigenous Offenders

\begin{tabular}{|c|c|c|c|}
\hline Measure & $\begin{array}{l}\text { Total Sample } \\
(N=1528)\end{array}$ & $\begin{array}{l}\text { Indigenous } \\
\text { Offenders } \\
(N=572)\end{array}$ & $\begin{array}{c}\text { Non- } \\
\text { Indigenous } \\
\text { Offenders } \\
(N=949)\end{array}$ \\
\hline & \multicolumn{3}{|c|}{ Custody Rating Scale } \\
\hline Gender-Informed Version 1 Continuous & $.28 * * *$ & $.30 * * *$ & $.18 * * *$ \\
\hline Gender-Informed Version 2 Continuous & $.28 * * *$ & $.29 * * *$ & $.18 * * *$ \\
\hline Gender-Informed Version 1 Trichotomized & $.27 * * *$ & $.28 * * *$ & $.17 * * *$ \\
\hline Gender-Informed Version 2 Trichotomized & $.27 * * *$ & $.27 * * *$ & $.17 * * *$ \\
\hline
\end{tabular}

Note. Gender-Informed Version 1 Continuous is the gender-informed scale that contains all 16 gender-informed predictors of institutional misconducts. Gender-Informed Version 2 Continuous is the gender-informed scale that excludes the DHS Historical Suicide predictor but contains the remaining 15 gender-informed predictors of institutional misconducts. Gender-Informed Version 1 Trichotomized is the trichotomized Gender-Informed Version 1 Continuous scale. GenderInformed Version 2 Trichotomized is the trichotomized Gender-Informed Version 2 Continuous scale.

$* p<.05 ; * * p<.01 ; * * * p<.001$

\section{Research question \#2: If gender-informed variables do emerge, can they yield incremental predictive validity above and beyond the Custody Rating Scale?}

To explore research question 2, two approaches were utilized. First, ROC statistics were computed to test the predictive accuracy of the Custody Rating Scale, Gender-Informed Version 1 Continuous, Gender-Informed Version 2 Continuous, Gender-Informed Version 1

Trichotomized, and Gender-Informed Version 2 Trichotomized. Formal ROC comparisons utilizing the method by Hanley and MacNeil (1983) were computed to determine if there were significant differences between the AUC values of the gender-informed scales and the Custody Rating Scale. Second, hierarchal cox regression analysis was computed for the gender-informed 
scales and Custody Rating Scale to determine if the gender-informed scales create incremental predictive validity above and beyond the Custody Rating Scale.

ROC Analysis. To determine the predictive accuracy of the Custody Rating Scale and gender-informed scales with any misconduct, ROC Analysis were computed ${ }^{2}$. Further, formal ROC Comparisons were also conducted to determine if there were statistically significant differences in AUC values for the Custody Rating Scale and gender-informed scales. It should be noted that for this analysis, the CRS recommendation of minimum, medium, and maximum was converted to '1' (minimum), '2' (medium), and '3' (maximum) due to SPSS Version 25 restricting ROC analysis with string variables. Results of the ROC statistics for the total sample, Indigenous offenders, and non-Indigenous offenders for any misconducts are provided in Table 18. Results of the AUC analysis for any misconducts revealed the CRS and gender-informed scales were above the .50 threshold; indicating predictive accuracy greater than chance. In accordance with Rice and Harris (2005), the results from the total sample revealed the GI-V1c and the GI-V2c scales correspond to large effect sizes. The CRS, GI-V1t scale, and GI-V2t scale correspond to medium effect sizes (Rice \& Harris, 2005). Results for the total sample indicate the gender-informed scales have greater AUC values than the CRS for any misconducts.

Results of ROC analysis revealed that for Indigenous offenders, the GI-V1c and GI-V2c scales had greater AUC values than the CRS. Further, the GI-V1c and GI-V2c scales correspond to large effect sizes, while the CRS corresponds to a medium effect size (Rice \& Harris, 2005). Results for the GI-V1t and GI-V2t scales demonstrated different findings, however, as the gender-informed scales did not demonstrate greater AUC values than the CRS. The GI-V1t scale, GI-V2t scale, and CRS all demonstrated medium effect sizes (Rice \& Harris, 2005).

\footnotetext{
${ }^{2}$ It should be noted that logistic regression analysis was also run for 6-month and 12-month follow-up periods and AUCs were computed. No substantial differences in results were found.
} 
For non-Indigenous offenders, the GI-V1c and GI-V2c scales had greater AUC values than the CRS. The GI-V1c and GI-V2c scales correspond to large effect sizes, while the CRS corresponds to a small effect size (Rice \& Harris, 2005). Results for the GI-V1t and GI-V2t scales demonstrated similar findings, as the GI-V1t and GI-V2t scales demonstrated greater AUC values than the CRS. The GI-V1t and GI-V2t scales correspond to medium effect sizes (Rice \& Harris, 2005). Details of the AUC analysis for any misconducts are presented in Table 19 below. ROC curves of the analysis for the total sample is also presented in Figures 4-7 in Appendix M, while ROC curves for Indigenous and non-Indigenous offenders are provided by Figures 8-15 in Appendix N. 
Table 19

ROC analysis of Institutional Misconduct with the Total Sample, Indigenous Offenders and non-Indigenous Offenders

\begin{tabular}{|c|c|c|c|c|c|c|c|c|c|}
\hline \multirow{2}{*}{ Measure } & \multicolumn{3}{|c|}{ Total Sample } & \multicolumn{3}{|c|}{ Indigenous Offenders } & \multicolumn{3}{|c|}{ Non-Indigenous Offenders } \\
\hline & AUC & $S E$ & $\mathrm{CI}$ & AUC & $S E$ & $\mathrm{CI}$ & AUC & $S E$ & $\mathrm{CI}$ \\
\hline \multicolumn{10}{|l|}{ Continuous GI Scales } \\
\hline Custody Rating Scale & .64 & $(.02)$ & {$[.61, .67]$} & .67 & $(.03)$ & {$[.62, .71]$} & .61 & $(.02)$ & {$[.57, .65]$} \\
\hline Gender-Informed Version 1 Continuous & .73 & $(.01)$ & {$[.70, .76]$} & .71 & $(.03)$ & {$[.66, .75]$} & .72 & $(.02)$ & {$[.68, .75]$} \\
\hline Gender-Informed Version 2 Continuous & .73 & $(.01)$ & {$[.70, .76]$} & .71 & $(.02)$ & {$[.66, .75]$} & .72 & $(.02)$ & {$[.68, .75]$} \\
\hline \multicolumn{10}{|l|}{ Trichotomized GI Scales } \\
\hline Custody Rating Scale & 64 & $(.02)$ & {$[.61, .67]$} & .67 & $(.03)$ & {$[.62, .71]$} & .61 & $(.02)$ & {$[.57, .65]$} \\
\hline Gender-Informed Version 1 Trichotomized & .69 & $(.01)$ & {$[.66, .72]$} & .66 & $(.03)$ & {$[.62, .71]$} & .68 & $(.02)$ & {$[.64, .71]$} \\
\hline Gender-Informed Version 2 Trichotomized & .69 & $(.01)$ & {$[.66, .71]$} & .65 & $(.03)$ & {$[.60, .70]$} & .67 & $(.02)$ & {$[.64, .71]$} \\
\hline
\end{tabular}

Note. Gender-Informed Version 1 Continuous is the gender-informed scale that contains all 16 gender-informed predictors of institutional misconducts. Gender-Informed Version 2 Continuous is the gender-informed scale that excludes the DHS Historical Suicide predictor but contains the remaining 15 gender-informed predictors of institutional misconducts. Gender-Informed Version 1 Trichotomized is the trichotomized Gender-Informed Version 1 Continuous scale. Gender-Informed Version 2 Trichotomized is the trichotomized Gender-Informed Version 2 Continuous scale.

$* p<.05 ; * * p<.01 ; * * * p<.001$ 
To determine if the gender-informed scales for any misconducts produce incremental predictive validity above and beyond the CRS, formal ROC comparisons were computed using the method developed by Hanley and McNeil (1983). In Test 1, the Gender-Informed Version 1 Continuous scale was compared with the CRS. Test 2 examined the Gender-Informed Version 2 Continuous scale and compared it with the CRS. Test 3 compared the Gender-Informed Version 1 Trichotomized scale to the CRS. Test 4 compared the Gender-Informed Version 2 Trichotomized scale with the CRS.

Results of the formal ROC comparisons are presented in Table 20. As illustrated in Table 20, the tests for the total sample indicated the difference in AUC values between the genderinformed scales and CRS were statistically significant as z-scores were greater than the critical value of 1.96 (two-tailed test) and confidence intervals did not contain zero. Similar findings were also demonstrated for non-Indigenous offenders. For Indigenous offenders, however, formal ROC comparisons indicated the difference in AUC values between the gender-informed scales and CRS were not statistically significant. Details are presented in Table 20 below. 
Table 20

Formal Comparison of ROC Curves with the Total Sample, Indigenous Offenders, and non-Indigenous Offenders

\begin{tabular}{|c|c|c|c|c|c|c|c|c|c|c|c|c|}
\hline \multirow{2}{*}{ Test } & \multicolumn{4}{|c|}{$\begin{array}{l}\text { Total Sample } \\
(N=1434)\end{array}$} & \multicolumn{4}{|c|}{$\begin{array}{l}\text { Indigenous Offenders } \\
\qquad(N=542)\end{array}$} & \multicolumn{4}{|c|}{$\begin{array}{l}\text { Non-Indigenous Offenders } \\
\qquad(N=886)\end{array}$} \\
\hline & $\begin{array}{l}\text { Difference } \\
\text { in Areas }\end{array}$ & $S E$ & $95 \% \mathrm{CI}$ & z score & $\begin{array}{l}\text { Difference } \\
\text { in Areas }\end{array}$ & $S E$ & $95 \%$ CI & z score & $\begin{array}{l}\text { Difference } \\
\text { in Areas }\end{array}$ & $S E$ & $95 \% \mathrm{CI}$ & z score \\
\hline Test 1 & .09 & $(.02)$ & $\begin{array}{l}{[.05} \\
.13]\end{array}$ & $4.82 * * *$ & .04 & $(.03)$ & $\begin{array}{c}{[-.02} \\
.10]\end{array}$ & 1.28 & .11 & $(.02)$ & $\begin{array}{l}{[.06,} \\
.16]\end{array}$ & $4.37 * * *$ \\
\hline Test 2 & .09 & $(.02)$ & $\begin{array}{l}{[.05} \\
.13]\end{array}$ & $4.79 * * *$ & .04 & $(.03)$ & $\begin{array}{l}{[-.02} \\
.10]\end{array}$ & 1.27 & .11 & $(.02)$ & $\begin{array}{l}{[.06} \\
.16]\end{array}$ & $4.38 * * *$ \\
\hline Test 3 & .05 & $(.02)$ & $\begin{array}{l}{[.02} \\
.08]\end{array}$ & $3.07 * *$ & .00 & $(.03)$ & $\begin{array}{c}{[-.06} \\
.07]\end{array}$ & .98 & .07 & $(.03)$ & $\begin{array}{l}{[.02} \\
.12]\end{array}$ & $2.65 * *$ \\
\hline Test 4 & .04 & $(.02)$ & $\begin{array}{l}{[.01} \\
.08]\end{array}$ & $2.65 * *$ & .02 & $(.03)$ & $\begin{array}{c}{[-.05} \\
.08]\end{array}$ & .50 & .06 & $(.03)$ & $\begin{array}{l}{[.02} \\
.11]\end{array}$ & $2.57^{*}$ \\
\hline
\end{tabular}

Note. Test 1 compares the Gender-Informed Version 1 Continuous scale with the Custody Rating Scale. Test 2 compares the GenderInformed Version 2 Continuous scale with the Custody Rating Scale. Test 3 compares the Gender-Informed Version 1 Trichotomized scale to the Custody Rating Scale. Test 4 compares the Gender-Informed Version 2 Trichotomized scale to the Custody Rating Scale. $* p<.05 ; * * p<.01 ; * * * p<.001$ 
Hierarchal Cox Regression Analysis. Hierarchal Cox regression survival analysis was also used to determine the contribution of and whether gender-informed factors produce incremental predictive validity beyond the CRS in the prediction of any misconducts. Four analyses were completed: two including the continuous gender-informed scales (GI-V1c and GIV2c), and two including the trichotomized gender-informed scales (GI-V1t and GI-V2t). Table 21 displays results for the continuous gender-informed scales with the CRS for the total sample, and Table 22 displays the results for the trichotomized gender-informed scales with the CRS for the total sample.

Table 21 presents the regression coefficients $(\beta)$, standard errors, Wald's statistic, Hazard ratio, confidence intervals, and chi-square statistic for the GI-V1c and GI-V2c scales. The CRS was entered first into the model, followed by the GI-V1c scale in the second block. For the second analysis, the CRS was entered first into the model, followed by the GI-V2c in the second block.

As demonstrated by Table 21, results indicate the CRS was a significant predictor of any misconducts in both analyses as the confidence intervals did not contain one. The addition of the GI-V1c scale in the second block did improve the model fit significantly as $\chi^{2}$ was statistically significant and the confidence intervals of the GI-V1c scale did not contain one. The GI-V1c scale was also statistically significant. Results were also similar for the addition of the GI-V2c. The addition of the GI-V2c scale in the second block also improved the model fit significantly as $\chi^{2}$ was statistically significant and confidence intervals of the GI-V2c scale did not contain one. Overall, the analysis supports the GI-V1c and GI-V2c contributed to the incremental predictive validity of the CRS. Details are provided in Table 21 below. 
Table 21

Hierarchal Cox Regression Analysis for the Custody Rating Scale and Continuous Genderinformed Scales for the Total Sample

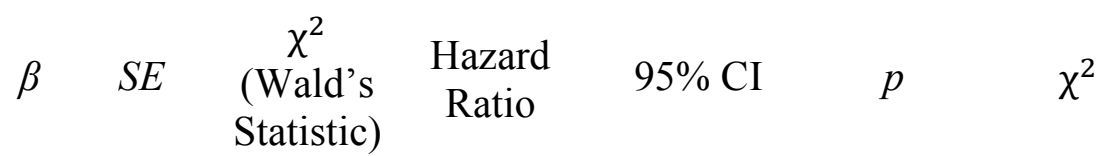

Gender-Informed Version 1 Scale

Block 1

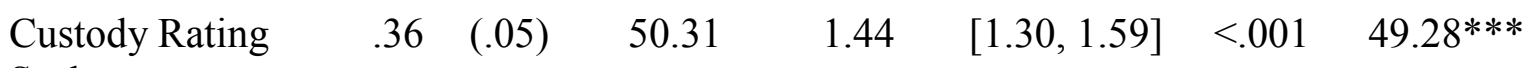

Scale

Block 2

$\begin{array}{llllllll}\begin{array}{l}\text { Custody Rating } \\ \text { Scale }\end{array} & .17 & (.05) & 10.64 & 1.19 & {[1.07,1.32]} & <.01 & 170.23 * * * \\ \begin{array}{l}\text { Gender-Informed } \\ \text { Version 1 }\end{array} & .12 & (.01) & 163.66 & 1.13 & {[1.11,1.15]} & <.001 & \\ \text { Continuous scale } & & & & & & & \end{array}$

Gender-Informed Version 2 Scale

\section{Block 1}

Custody Rating

Scale

\section{Block 2}

\begin{tabular}{|c|c|c|c|c|c|c|c|}
\hline $\begin{array}{l}\text { Custody Rating } \\
\text { Scale }\end{array}$ & .18 & $(.05)$ & 11.02 & 1.20 & {$[1.08,1.33]$} & $<.01$ & $168.63 * * *$ \\
\hline $\begin{array}{l}\text { Gender-Informed } \\
\text { Version } 2 \\
\text { Continuous scale }\end{array}$ & .13 & $(.01)$ & 161.78 & 1.13 & {$[1.11,1.16]$} & $<.001$ & \\
\hline
\end{tabular}

Note. Gender-Informed Version 1 Continuous is the gender-informed scale that contains all 16 gender-informed predictors of institutional misconducts. Gender-Informed Version 2 Continuous is the gender-informed scale that excludes the DHS Historical Suicide predictor but contains the remaining 15 gender-informed predictors of institutional misconducts.

$* p<.05 ; * * p<.01 ; * * * p<.001$

Table 22 presents the regression coefficients $(\beta)$, standard errors, Wald's statistic, Hazard ratio, confidence intervals, and chi-square statistic for the GI-V1t and GI-V2t scales. The CRS was entered first into the model, followed by GI-V1t scale in the second block. For the 
second analysis, the CRS was entered first into the model, followed by the GI-V2t scale in the second block.

As demonstrated by Table 22, results of the analysis indicates the CRS was a significant predictor of any misconducts for both analyses as confidence intervals did not contain one. The addition of the GI-V1t scale in the second block did improve the model fit significantly as $\chi^{2}$ was statistically significant. Results were similar for the GI-V2t scale as the addition of the GIV2t scale improved the model fit as $\chi^{2}$ was statistically significant. GI-V1t and GI-V2t scales were also statistically significant in the respective analysis as confidence intervals did not contain one. Both the GI-V1t and GI-V2t demonstrated statistically significant incremental predictive validity to the CRS. Details are provided in Table 22 below.

Table 22

Hierarchal Cox Regression Analysis for the Custody Rating Scale and Trichotomized Genderinformed Scales for the Total Sample

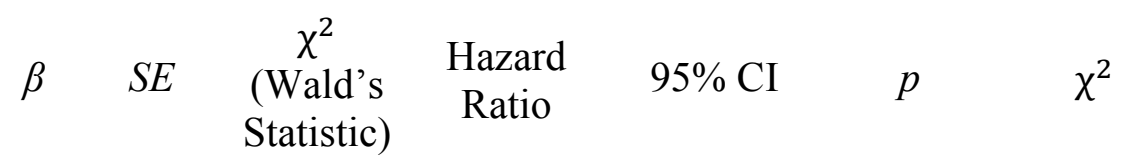

Gender-Informed Version 1 Scale

Block 1

$\begin{array}{llllllll}\text { Custody Rating } & .36 & (.05) & 50.31 & 1.44 & {[1.30,1.59]} & <.001 & 49.28 * * *\end{array}$

Scale

Block 2

$\begin{array}{lllllll}\text { Custody Rating } \quad .21 & (.05) & 15.97 & 1.24 & {[1.11,1.37]} & <.001 & 136.94 * * *\end{array}$

Scale

(Continued) 


\begin{tabular}{|c|c|c|c|c|c|c|c|}
\hline & $\beta$ & $S E$ & $\begin{array}{c}\chi^{2} \\
\text { (Wald's } \\
\text { Statistic) }\end{array}$ & $\begin{array}{c}\text { Hazard } \\
\text { Ratio }\end{array}$ & $95 \% \mathrm{CI}$ & $p$ & $\chi^{2}$ \\
\hline \multicolumn{8}{|c|}{ Gender-Informed Version 1 Scale } \\
\hline $\begin{array}{l}\text { Gender-Informed } \\
\text { Version } 1 \\
\text { Trichotomized }\end{array}$ & .61 & $(.05)$ & 139.90 & 1.84 & {$[1.67,2.04]$} & $<.001$ & \\
\hline \multicolumn{8}{|c|}{ Gender-Informed Version 2 Scale } \\
\hline $\begin{array}{l}\text { Block } 1 \\
\text { Custody Rating } \\
\text { Scale }\end{array}$ & .36 & $(.05)$ & 50.31 & 1.44 & {$[1.30,1.59]$} & $<.001$ & $49.28 * * *$ \\
\hline $\begin{array}{l}\text { Block } 2 \\
\text { Custody Rating } \\
\text { Scale }\end{array}$ & .22 & $(.05)$ & 16.95 & 1.25 & {$[1.12,1.38]$} & $<.001$ & $125.56 * * *$ \\
\hline $\begin{array}{l}\text { Gender-Informed } \\
\text { Version } 2 \\
\text { Trichotomized }\end{array}$ & .60 & $(.05)$ & 129.16 & 1.83 & {$[1.65,2.03]$} & $<.001$ & \\
\hline
\end{tabular}

Note. Gender-Informed Version 1 Trichotomized is the trichotomized Gender-Informed Version 1 Continuous scale. Gender-Informed Version 2 Trichotomized is the trichotomized GenderInformed Version 2 Continuous scale.

$* p<.05 ; * * p<.01 ; * * * p<.001$

Table 23 presents the regression coefficients $(\beta)$, standard errors, Wald's statistic, Hazard ratio, confidence intervals, and chi-square statistic for analysis with Indigenous and nonIndigenous offenders. Four analysis are presented in Table 23. For the first analysis, the CRS was entered first into the model, followed by GI-V1t scale in the second block. For the second analysis, the CRS was entered first into the model, followed by the GI-V2t scale in the second block. For the third analysis, the CRS was entered first in the model, followed by the GI-V1c scale in the second block. For the final analysis, the CRS was entered first in the model, followed by the GI-V2c scale in the second block.

As demonstrated by Table 23, results of the hierarchal cox regression analysis indicates the CRS was a significant predictor of any misconducts for all analysis in both Indigenous and non- 
Indigenous offenders as confidence intervals did not contain one. For both Indigenous and nonIndigenous offenders, the addition of the Gender-Informed Version 1 Trichotomized and Gender-Informed Version 2 Trichotmized scales in the second block improved the model fit as the $\chi^{2}$ was statistically significant and confidence intervals for the both scales did not contain one. The GI-V1t and GI-V2t scales had a significant contribution to the incremental predictive validity of the CRS.

For Indigenous and non-Indigenous offenders, the addition of the Gender-Informed Version 1 Continuous scale and the Gender-Informed Version 2 Continuous in the second block also improved the model fit as the $\chi^{2}$ was statistically significant and the confidence intervals of each scale did not contain one. Both the GI-V1c and GI-V2c demonstrated significant contribution to the incremental predictive validity of the CRS. Details are provided in Table 23 below. 
Table 23

Hierarchal Cox Regression for the Custody Rating Scale and Trichotomized Gender-informed Scales by Indigenous Status

\begin{tabular}{|c|c|c|c|c|c|c|c|c|c|c|c|c|c|c|}
\hline & \multicolumn{7}{|c|}{ Indigenous Offenders } & \multicolumn{7}{|c|}{ Non-Indigenous Offenders } \\
\hline & $\beta$ & $S E$ & $\begin{array}{c}\chi^{2} \\
\text { (Wald's } \\
\text { Statistic) }\end{array}$ & $\begin{array}{c}\text { Hazard } \\
\text { Ratio }\end{array}$ & $\begin{array}{c}95 \% \\
\text { CI }\end{array}$ & $p$ & $\chi^{2}$ & $\beta$ & $S E$ & $\begin{array}{c}\chi^{2} \\
\text { (Wald's } \\
\text { Statistic) }\end{array}$ & $\begin{array}{c}\text { Hazard } \\
\text { Ratio }\end{array}$ & $\begin{array}{c}95 \% \\
\text { CI }\end{array}$ & $p$ & $\chi^{2}$ \\
\hline \multicolumn{15}{|c|}{ Gender-Informed Version 1 Trichotomized } \\
\hline $\begin{array}{l}\text { Block } 1 \\
\text { Custody Rating } \\
\text { Scale }\end{array}$ & .34 & $(.08)$ & 19.04 & 1.40 & $\begin{array}{c}{[1.21,} \\
1.63]\end{array}$ & $<.001$ & $18.95 * * *$ & .29 & $(.07)$ & 17.11 & 1.34 & $\begin{array}{c}{[1.17,} \\
1.54]\end{array}$ & $<.001$ & $16.67 * * *$ \\
\hline $\begin{array}{l}\text { Block } 2 \\
\text { Custody Rating } \\
\text { Scale }\end{array}$ & .23 & $(.08)$ & 8.16 & 1.26 & $\begin{array}{c}{[1.08,} \\
1.47]\end{array}$ & $<.01$ & $21.79 * * *$ & .23 & $(.07)$ & 9.27 & 1.25 & $\begin{array}{c}{[1.08,} \\
1.45]\end{array}$ & $<.01$ & $104.45 * * *$ \\
\hline GI-V1t & .38 & $(.08)$ & 21.67 & 1.47 & $\begin{array}{c}{[1.25,} \\
1.73] \\
\end{array}$ & $<.001$ & & .78 & $(.07)$ & 110.64 & 2.18 & $\begin{array}{l}{[1.89} \\
2.52]\end{array}$ & $<.001$ & \\
\hline \multicolumn{15}{|c|}{ Gender-Informed Version 2 Trichotomized } \\
\hline $\begin{array}{l}\text { Block 1 } \\
\text { Custody Rating } \\
\text { Scale }\end{array}$ & .34 & $(.08)$ & 19.04 & 1.40 & $\begin{array}{c}{[1.21,} \\
1.63]\end{array}$ & $<.001$ & $18.95 * * *$ & .29 & $(.07)$ & 17.11 & 1.34 & $\begin{array}{c}{[1.17,} \\
1.54]\end{array}$ & $<.001$ & $16.67 * * *$ \\
\hline $\begin{array}{l}\text { Block } 2 \\
\text { Custody Rating } \\
\text { Scale }\end{array}$ & .24 & $(.08)$ & 9.21 & 1.28 & $\begin{array}{l}{[1.09,} \\
1.49]\end{array}$ & $<.01$ & $17.90 * * *$ & .22 & $(.07)$ & 8.97 & 1.25 & $\begin{array}{l}{[1.08,} \\
1.44]\end{array}$ & $<.01$ & $99.08 * * *$ \\
\hline GI-V2t & .36 & $(.08)$ & 17.84 & 1.43 & $\begin{array}{c}{[1.21,} \\
1.68]\end{array}$ & $<.001$ & & .78 & $(.08)$ & 105.90 & 2.18 & $\begin{array}{l}{[1.88,} \\
2.53]\end{array}$ & $<.001$ & \\
\hline Gender-Informed V & ion 1 & Continu & & & & & & & & & & & & \\
\hline $\begin{array}{l}\text { Block 1 } \\
\text { Custody Rating } \\
\text { Scale }\end{array}$ & .34 & $(.08)$ & 19.04 & 1.40 & $\begin{array}{c}{[1.21,} \\
1.63]\end{array}$ & $<.001$ & $18.95 * * *$ & .29 & $(.07)$ & 17.11 & 1.34 & $\begin{array}{c}{[1.17} \\
1.54]\end{array}$ & $<.001$ & $16.67 * * *$ \\
\hline $\begin{array}{l}\text { Block } 2 \\
\text { Custody Rating } \\
\text { Scale }\end{array}$ & .20 & $(.08)$ & 5.83 & 1.22 & $\begin{array}{c}{[1.04,} \\
1.42]\end{array}$ & $<.05$ & $32.37 * * *$ & .18 & $(.07)$ & 5.98 & 1.20 & $\begin{array}{c}{[1.04,} \\
1.38]\end{array}$ & $<.05$ & $121.52 * * *$ \\
\hline GI-V1c & .09 & $(.02)$ & 30.94 & 1.10 & $\begin{array}{c}{[1.06,} \\
1.13]\end{array}$ & $<.001$ & & .14 & $(.01)$ & 120.12 & 1.15 & $\begin{array}{c}{[1.12,} \\
1.18]\end{array}$ & $<.001$ & Continued) \\
\hline
\end{tabular}




\begin{tabular}{|c|c|c|c|c|c|c|c|c|c|c|c|c|c|c|}
\hline & \multicolumn{7}{|c|}{ Indigenous Offenders } & \multicolumn{7}{|c|}{ Non-Indigenous Offenders } \\
\hline & $\beta$ & $S E$ & $\begin{array}{c}\chi^{2} \\
\text { (Wald's } \\
\text { Statistic) }\end{array}$ & $\begin{array}{c}\text { Hazard } \\
\text { Ratio }\end{array}$ & $\begin{array}{c}95 \% \\
\text { CI }\end{array}$ & $p$ & $\chi^{2}$ & $\beta$ & $S E$ & $\begin{array}{c}\chi^{2} \\
\text { (Wald's } \\
\text { Statistic) }\end{array}$ & $\begin{array}{c}\text { Hazard } \\
\text { Ratio }\end{array}$ & $\begin{array}{c}95 \% \\
\text { CI }\end{array}$ & $p$ & $\chi^{2}$ \\
\hline \multicolumn{15}{|c|}{ Gender-Informed Version 2 Continuous } \\
\hline $\begin{array}{l}\text { Block } 1 \\
\text { Custody } \\
\text { Rating Scale }\end{array}$ & .34 & $(.08)$ & 19.04 & 1.40 & $\begin{array}{l}{[1.21} \\
1.63]\end{array}$ & $<.001$ & $18.95 * * *$ & .29 & $(.07)$ & 17.11 & 1.34 & $\begin{array}{c}{[1.17} \\
1.54]\end{array}$ & $<.001$ & $16.67 * * *$ \\
\hline $\begin{array}{l}\text { Block } 2 \\
\quad \text { Custody } \\
\text { Rating Scale }\end{array}$ & .20 & $(.08)$ & 5.93 & 1.22 & $\begin{array}{c}{[1.04,} \\
1.43]\end{array}$ & $<.05$ & $32.99 * * *$ & .19 & $(.07)$ & 6.32 & 1.20 & $\begin{array}{c}{[1.04,} \\
1.39]\end{array}$ & $<.05$ & $118.08 * * *$ \\
\hline GI-V2c & .10 & $(.02)$ & 31.31 & 1.10 & $\begin{array}{r}{[1.06,} \\
1.14]\end{array}$ & $<.001$ & & .15 & $(.01)$ & 117.18 & 1.16 & $\begin{array}{c}{[1.13,} \\
1.19]\end{array}$ & $<.001$ & \\
\hline
\end{tabular}

$* p<.05 ; * * p<.01 ; * * * p<.001$ 


\section{Discussion}

The purpose of the current study was to explore whether the proposed gender-informed domains (mental health, substance abuse, relationship dysfunction, parental/family issues, victimization, and personal/emotional issues) would predict institutional misconducts in women offenders and Indigenous women offenders. Further, the purpose of the current study was also to explore whether the gender-informed variables would create incremental predictive validity to the Custody Rating Scale (CRS) in women offenders and Indigenous women offenders. To date, this was the first study to utilize a women-centered theoretical approach to explore the addition of gender-informed variables to the CRS. The majority of forensic assessments have been developed and validated primarily with male offenders (Salisbury et al., 2009); including the use of the CRS for classification of offenders. Thus, the current study has provided the opportunity for the consideration of a woman-centered approach and factors that may be more pertinent to institutional misbehaviours in women offenders. Findings from this study and the implications in regards to the hypothesis will be discussed.

\section{Gender-Informed Predictors of Institutional Misconduct in Women Offenders}

For the first research question, it was hypothesized that the gender-informed domains mental health, substance abuse, relationship dysfunction, parental/family issues, victimization, and personal/emotional factors would be predictive of institutional misconducts. The personal/emotional factors were hypothesized to be weak predictors in accordance to the literature. In order to test this hypothesis, cox regression survival analysis were computed for all the predictors in each domain for any misconducts. Based on the results, it was concluded that

this hypothesis was partially supported. Items from each gender-informed domain were found to be predictive of receiving any institutional misconduct charge. 
Despite that items from each gender-informed domain emerged as predictive for institutional misconducts, some important aspects should be noted. First, the hypothesis that the mental health, substance abuse, relationship dysfunction, parental/family issues, and victimization domains would be predictive of institutional misconduct in this sample of women offenders was supported. These results are consistent with prior research, as mental health (Reidy \& Sorensen, 2018; Houser \& Welsh, 2014), substance abuse (Davidson et al., 2016), relationship dysfunction (Van Voorhis et al., 2010), parental/family issues (Celinska \& Sung, 2014) and victimization (Celinska \& Sung, 2014) were found to be predictors of institutional misconduct in this sample of women offenders.

However, the hypothesis that the personal/emotional domain would be weak a predictor of institutional misconducts was not supported. In fact, results suggest that a greater proportion of items from the personal/emotional domain in comparison to the other domains were significant predictors for any misconducts. Further, the items, 'frequently feels intense anger' and 'has low frustration tolerance' were found to be strong predictors of any misconducts. The item 'frequently feels intense anger' being predictive of institutional misconducts is consistent with prior research, as anger has been implicated as a predictor of institutional misconducts in offender populations (McDermott, Edens, Quanbeck, Busse \& Scott, 2008) and women offenders (Van Voorhis et al., 2010).

Despite that prior research has found that overall, the personal/emotional domain is a weak predictor of institutional misconduct in women offenders (e.g., Rettinger \& Andrews, 2010), there may be an explanation for the current findings. For instance, prior studies have found that for women, experiencing anger, poor communication, and difficulties with emotional regulation have contributed to women's psychological and physical aggression in interpersonal 
relationships (Hettrich \& O'Leary, 2007; Shorey, Cornelius \& Idema, 2011). Further, Hornsveld et al. (2018) conducted a study in a sample of 59 female offenders and 170 male offenders convicted of a violent crime to examine personality traits and problem behaviours relating to aggressive behaviours. The researchers found that, in comparison to male offenders, the female offenders had higher scores on trait anger $(F(2,217)=4.55, p=.035, \eta 2=.02)$ and neuroticism $(F(2,218)=8.26, p=.004, \eta 2=.04)$; albeit the effect sizes were small. Comparing the outpatients and detainees groups for male and female offenders, a consistent difference that emerged in the violent offenders was greater emotional instability (greater scores in neuroticism) in the female offenders. Emotional instability and dysregulation may be a contributing factor to women offenders' aggressive behaviours.

A study conducted by Moore et al. (2018) examined the relation between borderline personality disorder (BPD), aggression toward others, victimization, and institutional misconduct in a sample of 184 male and female prisoners. BPD is characterized by instability in affective regulation, impulse control, self-image, and interpersonal relationships (Lieb, Zanarini, Schmahl, Linehan \& Bohus, 2004); and also consists of severe impairment in functioning and a high risk of suicide (Leichsenring, Leibing, Kruse, New \& Leweke, 2011). Moore et al. (2018) found that individuals with BPD were more likely than individuals without BPD to self-report disciplinary infractions (defined as fighting, having arguments with prison staff, receiving disciplinary action, or being put in isolation) during their incarceration. Moore and colleagues (2018) point out that the extreme display of emotion and behavioural issues from poor tolerance to emotional distress may prompt individuals with BPD to disobey orders from correctional staff. As such, women offenders that experience difficulties in the personal/emotional domain (e.g., having low frustration tolerance, experiencing intense anger, difficulties solving interpersonal problems) 
likely also experience difficulty tolerating emotional stress which prompts these women offenders to engage in institutional misconducts.

It is also interesting to note the item 'assertiveness skills are limited' had a negative beta value and subsequently high scores on this item predicted a lesser likelihood of institutional misconduct. Assertiveness is conceptualized as the tendency for an individual to actively protect, pursue, and effectively communicate their own self-interests (Ames \& Flynn, 2007). One explanation for this finding may be that the item captures the concept of self-efficacy. According to Salisbury et al. (2009), self-efficacy is the confidence an individual has in achieving specific goals. Despite that there is little research in regards to self-efficacy and institutional misconducts, Salisbury et al. (2009) found self-efficacy increased the likelihood of prison misconducts but decreased the likelihood of community recidivism. The authors hypothesize that self-efficacy is a potential risk factor for women in the institutional setting as opposed to in the community; as women that are self-efficacious may challenge prison authorities more often and receive more citations from correctional staff. It is possible that women offenders that display confidence to defend their own self-interests (assertiveness) may challenge prison authorities and other inmates more frequently, leading to greater likelihood of citations for misconduct and engaging in misconducts.

It is also possible that women offenders are receiving institutional charges by correctional officers for assertive behaviour. Assertiveness may also be implicated in engaging in aggressive behaviours in offender populations. Ireland (2002) found in a sample of 285 men and 217 women offenders that assertiveness is a factor of bullying behaviour in offenders. Significant, positive correlations were found between overall assertiveness and overall bullying $(r=.13$, $p<.004)$, indirect bullying $(r=.11, p<.01)$, and direct bullying $(r=.12, p<.01)$. Further, 
significant positive correlations were also found between argumentativeness/combativeness and overall bullying $(r=.28, p<.001)$, indirect bullying $(r=.23, p<.001)$, and direct bullying $(r=$ $.25, p<.001)$. As such, women offenders that display more assertive behaviours may be more likely to display combative and argumentative behaviours that escalate problematic situations with correctional staff and other inmates. The assertive behaviour may be perceived as being defiant or confrontational, which may lead to greater likelihood of aggressive behaviours towards others and subsequent institutional charges.

Figures 1 and 2 also demonstrate support for hypothesis 1 . Women offenders that scored in the high group on gender-informed predictors (had greater scores on the gender-informed predictors that emerged) had lesser time to failure, and were more likely to engage in misconducts than women offenders in the medium and low groups. Women offenders that were in the medium group also had lesser time to failure and were more likely to engage in misconducts than women offenders in the low group. As such, women offenders that demonstrated more concerns in the domains of mental health, substance abuse, relationship dysfunction, parental/family issues, victimization, and personal/emotional factors engaged in more misconducts and had lesser time to failure. Thus, these domains are predictive of any misconducts in this sample of women offenders.

In comparison to Figure 3 of the CRS, Figures 1 and 2 also reveal the Gender-Informed Version 1 Trichotomized and Gender-Informed Version 2 Trichotomized scales demonstrate improvement in distinguishing the medium group from the high group; in comparison to the CRS distinction between medium and maximum security. This is illustrated by the overlap in the survival curves of medium and maximum security offenders in accordance to the CRS (Figure 3) and the lack of overlap in the medium and high group for the GI-V1t and GI-V2t scales (Figures 
1 and 2). Overall, the figures reveal that both the CRS and gender-informed scales distinguish offenders that are more likely to engage in misconducts and have less time to failure.

Further, bivariate correlations examining the relationship between the CRS and genderinformed scales also demonstrated interesting findings. All gender-informed scales had a strong relation to the CRS, providing support for the convergent validity of the gender-informed scales. Murphy and Davidshofer (2005) indicate a common method to assessing convergent validity (also construct validity) is to correlate measures to determine how well scores provide a good measure of a specific construct. However, it is interesting to note that the relationship between the gender-informed scales and the CRS was weaker for non-Indigenous offenders; albeit still strong. As such, the strong positive correlations of the gender-informed scales with the CRS demonstrate support for the gender-informed scales to adequately measure likelihood of misconducts and security designations in women offenders.

These analysis provide support that items from each gender-informed domain were predictive of institutional misconducts in this sample of women offenders. The analysis also provides support for the importance of each gender-informed domain in predicting institutional misconducts for women offenders and the ability to distinguish women offenders that are more likely to incur institutional charges.

\section{Incremental Predictive Validity of Gender-informed Factors to the CRS}

For the second research question, it was hypothesized the gender-informed domains would provide incremental predictive validity above and beyond the Custody Rating Scale. Overall, results from the analyses provide support for hypothesis two that the gender-informed domains did yield incremental predictive validity above and beyond the CRS. It is not to argue that the CRS is not predictive of institutional misconducts in women or does not offer usefulness 
in the initial security classification of women offender populations. The CRS did demonstrate strong associations to any institutional misconducts and adequate predictive accuracy as the AUC values were consistent with medium effect sizes.

The argument is the overall analysis provides support for the inclusion of genderinformed factors to current gender-neutral approaches. For instance, bivariate correlations for the total sample provided support for the association between the gender-informed domains and engaging in institutional misconducts. It is interesting to note that overall, the gender-informed scales demonstrated stronger associations to institutional misconducts than the CRS for the total sample, Indigenous women offenders, and non-Indigenous women offenders.

All gender-informed scales for the total sample demonstrated greater AUC values than the CRS, supporting greater predictive accuracy of the gender-informed domains for institutional misconducts. To determine if these differences were meaningful, ROC comparisons were computed and results supported a meaningful difference between the gender-informed domains and CRS. The gender-informed scales demonstrate improved predictive accuracy in comparison to the CRS and ROC comparisons provide support for the incremental predictive validity of the gender-informed scales.

To further test for the incremental predictive validity of the gender-informed scales, hierarchal cox regression was also used. The addition of the gender-informed scales in the total sample provided better model fit when included in the analysis, and each gender-informed scale demonstrated significance as confidence intervals did not contain one. Thus, the genderinformed scales contributing to the incremental predictive validity above and beyond the CRS was supported. These analyses indicate that for the overall sample, gender-informed factors by themselves provide strong predictive accuracy for the prediction of institutional misconducts and 
when added to the CRS they enhance predictive validity. This finding is consistent with prior research that found the addition of gender-informed factors to gender-neutral assessments contributed to the incremental predictive validity of institutional misconduct in women offenders (Van Voorhis et al., 2010; Wright et al., 2007). The current analysis provides additional support for inclusion of gender-informed factors to gender-neutral assessments - in particular the CRS. Incremental Predictive Validity of Gender-Informed Factors to the CRS and Indigenous

\section{Women Offenders}

The analyses were also run to determine if these findings were consistent for Indigenous and non-Indigenous offenders. Consideration must be given to Indigenous offenders in current research as Indigenous peoples are over-represented in the correctional system (CSC, 2017), and the over-representation is more pronounced in Indigenous women offenders as they comprise one out of every three incarcerated women (Wesley, 2012). It is imperative to explore if genderinformed factors predict institutional misconducts and yield incremental predictive validity to the CRS in Indigenous women offenders.

Analysis of the predictive accuracy of the gender-informed scales was supported as AUCs corresponded to medium and large effect sizes (Rice \& Harris, 2005) for Indigenous offenders. Predictive accuracy of the gender-informed scales was also supported for nonIndigenous offenders, as AUCs also corresponded to medium and large effect sizes (Rice \& Harris, 2005). Thus, the gender-informed scales were found to be predictive of receiving an institutional charge for Indigenous and non-Indigenous women offenders.

However, the ROC comparisons and hierarchical cox regression analysis that was used to test incremental predictive validity demonstrated mixed findings for Indigenous women offenders. Results from the ROC comparisons for Indigenous women offenders demonstrated the 
difference between the AUC values for all of the gender-informed scales and the CRS were not statistically significant. For the hierarchical cox regression analysis, the gender-informed scales (GI-V1t, GI-V2t, GI-V1c and GI-V2c) improved the model fit and contributed incremental predictive validity when added to the CRS.

Findings for non-Indigenous offenders were more consistent, as overall analysis provided support for the utilization of gender-informed factors in classification for non-Indigenous women offenders. The results from ROC comparisons for non-Indigenous offenders revealed the difference in AUC values between all of the gender-informed scales with the CRS was statistically significant. Further, overall analysis of the hierarchical cox regression provided support for the incremental predictive validity of the gender-informed scales as addition of these scales were significant and improved the model fit. To the author's knowledge, no prior studies have examined the predictive accuracy of gender-informed factors with non-Indigenous and Indigenous women offenders, and the inclusion of gender-informed factors to classification assessments such as the CRS.

However, Barnum and Gobeil (2012) did an exploratory analysis of the inclusion of criminogenic needs to the CRS in Indigenous and non-Indigenous women offenders. Despite that gender-informed factors specifically were not investigated, there is some relevance to the criminogenic needs that were studied to the gender-informed factors. Barnum and Gobeil (2012) examined the associations between the DFIA domains (Substance Abuse, Marital/Family, Employment, Associates, Community Functioning, and Personal/Emotional) and minor/serious institutional charges, minor/serious incidents, release type, any return, and any return with offence for Indigenous and non-Indigenous women offenders. They found the DFIA domains most pertinent to gender-informed domains in this study (Substance Abuse, Marital/Family, and 
Personal/Emotional) had moderate to strong associations with institutional outcomes for both Indigenous and non-Indigenous women. However, when examining the overall pattern of findings for all outcomes in Indigenous and non-Indigenous women offenders, Barnum and Gobeil (212) found no clear pattern emerged for institutional behaviors in Indigenous offenders. Barnum and Gobeil (2012) suggest the level of need in the DFIA domains may contribute to security classification in non-Indigenous offenders as they noted the DFIA domains (with the exception of Employment and Associates) demonstrated moderate relationships with major institutional incidents.

Taken collectively, some caution is needed for the use of gender-informed domains in classification with Indigenous women offenders. The mixed findings of this study and lack of prior research on gender-informed predictors with Indigenous women offenders does question the evidence for inclusion of gender-informed domains to the CRS for Indigenous women offenders. The results from the overall sample and non-Indigenous women offenders did provide more consistent findings for the addition of gender-informed domains to gender-neutral classification assessments.

\section{Implications of the Current Study}

The current study contributes to the literature by exploring potential predictors of institutional misconduct for women offenders. Research is still limited in using female-only samples to determine predictors of institutional misconducts in women offenders. The current

study also contributed to the lack of research of gender-informed predictors to the addition of gender-neutral assessments, and contributed to the predominately male-centric approach of research with women offenders by utilizing a theoretically driven women-centered approach. This study also utilized more robust statistical analysis to explore the predictive validity of 
gender-informed domains and the potential contribution of gender-informed domains to the CRS in women offenders. Prior studies by Van Voorhis et al. (2010) and Wright et al. (2007) utilized correlational analysis to determine the incremental predictive validity of the addition of genderinformed factors to gender-neutral assessments. The current study used ROC comparisons and hierarchal cox regression to determine the incremental predictive validity of addition of genderinformed domains to the CRS. Further, this study also explored the analysis with Indigenous women offenders as well, which has been neglected in the current literature.

Overall, findings were supportive of the hypotheses and encouraging for the inclusion of gender-informed domains to classification of women offenders. It is not the intention to argue that gender-neutral assessments are not useful with women offenders, as prior research has supported and validated the use of the CRS with women offenders (Barnum \& Gobeil, 2012). However, the addition of gender-informed predictors to the CRS can enhance the predictive accuracy of the CRS in women offenders. The findings in this study have implications for Correctional Service of Canada and the classification process for women offenders. Even though the CRS demonstrated 'acceptable' predictive ability for any misconduct in this sample of women offenders, the majority of the gender-informed scales had greater predictive accuracy than the CRS. Not only did the gender-informed scales demonstrate greater predictive ability, but overall the addition of gender-informed factors improved the incremental predictive validity of the CRS for women offenders. Modification to the CRS for classification of women offenders is supported by the findings in this study.

The overall analysis for Indigenous women offenders supported the addition genderinformed predictors to the CRS as the gender-informed scales produced incremental predictive validity to the CRS. However, some caution should be noted for the analysis with Indigenous 
women offenders. Despite that most of the gender-informed scales provided incremental predictive validity to the CRS, ROC comparisons for all gender-informed scales demonstrated an inability to contribute incremental predictive validity to the CRS. Further, the GI-V2t had slightly lower AUC values than the CRS. Due to the over-representation of Indigenous women offenders in the correctional system (CSC, 2017b), it is imperative to be cautious with how Correctional Service of Canada classifies Indigenous women offenders.

Concerns surrounding the use of actuarial assessments with Indigenous peoples warrants caution. The case of Ewert v. Canada (2015) brought forth issues regarding the use of actuarial risk assessments with Indigenous peoples. The case concluded the research supporting the use of these actuarial measures with Indigenous offenders was inadequate, and the measures were susceptible to cultural bias (Shepherd \& Lewis-Fernandez, 2016). It is imperative to obtain more substantial, rigorous evidence for the use of gender-informed factors with Indigenous women offenders before concluding that gender-informed factors are applicable with Indigenous women.

It should be noted the creation of two versions for the gender-informed scales that either included or excluded the DHS Historical Suicide also has implications. The overall analysis between the two versions for the continuous scales (GI-V1c versus GI-V2c) and the trichotomized scales (GI-V1t versus GI-V2t) did not produce any substantial differences in the findings. Since the inclusion of the DHS Historical Suicide item did not produce any differences in the analysis, it is recommended to exclude the DHS Historical Suicide item from the genderinformed scales. The DHS Historical Suicide item captures previous suicide/self-harm behaviours and serious thoughts of suicide (Mills \& Kroner, 2008). The inclusion of the DHS Historical Suicide item can penalize women for having prior self-harm/suicidal behaviours and 
serious thoughts of suicide. This is problematic and has the potential to designate women to higher security levels due to prior suicidal behaviours or serious thoughts of suicide.

Taken collectively, the findings provide support for the addition of gender-informed predictors to the CRS in women offenders, non-Indigenous women offenders, and Indigenous offenders. Further, the study also provides support for gender-informed perspective in general in women offenders. The CRS is considered gender-neutral, and the predictive accuracy of this instrument was enhanced with gender-informed predictors. The addition of gender-informed predictors should be considered for other gender-neutral assessments that have also been normed and validated with male samples. It is possible that these factors are applicable to women offender populations in other contexts than classification (e.g., assessing risk of recidivism).

\section{Limitations and Future Research}

There are a number of limitations in the current study that need to be addressed. The first limitation that should be addressed is the use of archival data in the study. Despite that archival data can provide extensive information that is easier for researchers to gather, it also can lead to bias as some information may be selected to be stored in archival databases but others may not be included. Further, archival data may include incomplete information or records that are missing (Shaughnessy, Zechmeister \& Zechmeister, 2012). The database of the current study did have some measures (e.g., Mental Health Indicators) with substantial missing values, thus they had to be dropped from inclusion in the analysis. The most concerning limitation to the use of archival data is the lack of causal inferences that can be made, thus leading to the potential for another third variable to explain relationships between variables (Shaughnessy et al., 2012). Despite that the findings did provide support for gender-informed factors in women offenders, it is not established that greater issues of gender-informed factors in women offenders (e.g., having 
relationship dysfunction) causes institutional misconducts. Thus, caution in interpretation of the findings of this study is warranted.

The second limitation to this study is that some of the gender-informed domains in this study differed from prior research, specifically, from Van Voorhis et al. (2010) and Wright et al. (2007). For instance, Van Voorhis et al. (2010) and Wright et al. (2007) incorporate the genderinformed domain of poverty and self-efficacy in their analysis. The current study was limited in the ability to provide items that directly coincided with these gender-informed domains.

However, based on prior research from Van Voorhis et al. (2010) and Wright et al. (2007), the Personal/Emotional domain includes items that coincide with self-esteem and self-efficacy. As such, items such as 'gives up easily when challenged,' 'assertiveness skills are limited,' and 'has difficulty solving interpersonal problems' were selected to best capture issues with self-esteem and self-efficacy. Despite attempts to align items in the dataset with the prior gender-informed research, data was limited by previously collected archival data.

A third limitation to the study is the lack of a male sample comparison group. A male comparison group would assist in deciphering if certain predictors are applicable for both men and women equally, are stronger for one gender in comparison to the other, or are pertinent for only one gender (Scott, 2017). Despite that the current study found support for gender-informed domains to be predictive of institutional misconduct in women offenders, the lack of a male comparison group limits the ability to determine if these predictors are relevant to women only. Feminist perspectives, especially pathways theory, tends to focus on victimization at young ages leading to antisocial behaviours in women (Kruttschnitt, 2016). However, victimization is not a sole experience of women offenders (Kruttschnitt, 2016), and may also be implicated in institutional misconducts in men offenders. For instance, Cain, Steiner, Wright and Meade 
(2016) found that prior to incarceration, being physically assaulted by a non-stranger had a stronger effect for men offenders in comparison to women offenders for engaging in violent misconducts. Without a male comparison group, the relative extent of how the gender-informed domains are pertinent to women cannot be established.

A fourth limitation to the study is the use of the substance abuse domain as genderinformed. Although Van Voorhis et al. (2010) and Wright et al. (2007) have indicated the substance abuse can be conceptualized as a gender-responsive need due to substance abuse having unique effects on women, the domain still warrants caution. Substance abuse is also incorporated as a predictor for recidivism and criminal behaviours in gender-neutral theoretical perspectives of assessment measures (Andrews et al., 2012). As such, until further research explores the extent of substance abuse (as well as other gender-informed domains) as a predictor of institutional misconducts in men and women offenders, interpretation of substance abuse as a gender-informed construct is limited.

The final limitation to the study concerns the amount of missing data in that dataset. The t-tests and chi-square tests found some interesting patterns that emerged in the missing data. The DHS indicators and GSI subscale of the Brief Symptom Inventory demonstrated significant patterns with the date CoMHISS was administered, date DFIA-R was administered, and date WCASA was administered; and the DHS Indicators only demonstrated significant patterns for the version number of CoMHISS. Further, Indigenous women were more likely to have missing values on the DHS indicators and GSI subscale of the Brief Symptom Inventory than nonIndigenous women. The DFIA-R PER06 item, 'gives up easily when challenged' demonstrated some significant patterns with the Personal/Emotional predictors of the DFIA-R. The finding that the DFIA-R PER06 item 'gives up easily when challenged' demonstrated some significant 
patterns with other Personal/Emotional domain predictors is not surprising. It is possible the missing values for this item may be attributed to a general lack of responding on the Personal/Emotional domain in general. Individuals that were less likely to respond in general on the Personal/Emotional domain may be more likely to not respond on this particular item.

One possible explanation for the missing data may be due to the implementation and administration of CoMHISS to federal offenders. CoMHISS was developed and implemented by CSC in 2009 (Stewart, Archambault \& Wilton, 2013). The first version of CoMHISS included the GSI subscale of the Brief Symptom Inventory and the Depression, Hopelessness and Suicide Screening Form; while CSC created version 2 in 2012 which included additional screening for cognitive functioning, mental health and ADHD (Martin, Wamboldt, O'Connor, Fortier \& Simpson, 2013). Women offenders with a Warrant of Committal after 2012 would have been given version 2 of the CoMHISS that included the Mental Health Indicators, GAMA and ASRS. Women offenders that had a Warrant of Committal before 2012 would have been given version 1 and data for the Mental Health Indicators, GAMA and ASRS would be missing for this cohort.

The finding that missing data was predominant for Indigenous women offenders is concerning. The missing data for Indigenous women offenders may be explained by the actual screening process for incoming federal offenders. Martin, Crocker, Potter, Wells, Grace and Colman (2018) conducted a study that examined the screening process of CoMHISS in Canadian prisons. There were 7965 admissions from the Canadian correctional system that was included in the study. Martin et al. (2018) found that out of the total of women offenders in the sample $(N=$ 510), $24.5 \%$ of these women were not screened with CoMHISS. Further, Martin et al. (2018) also found that for Indigenous offenders $(N=1860), 22.2 \%$ were also not screened with CoMHISS. Unfortunately, the Indigenous offender analysis was not disaggregated by gender. It 
may be that a substantial portion of missing data in this study sample is due to a lack of screening and administration of CoMHISS to these women offenders with missing CoMHISS data. Further, CoMHISS is a self-administered assessment tool. Potential reasons CoMHISS as a self-administered assessment tool may contribute to the missing data in Indigenous women may include a lack of trust to fully disclose symptoms and the length of CoMHISS may impede ability to complete all questions. Future research should explore the potential factors that may be contributing to missing data in CoMHISS information for Indigenous women offenders.

It should be noted, however, that due to the concern of missing data, two genderinformed scales were created. The decision to exclude the DHS Historical Suicide item was due to the amount of missing data in this item. Since the overall analysis did not differ between the gender-informed scales that excluded the DHS Historical Suicide item from the gender-informed scales the included the DHS Historical Suicide item, concerns regarding missing data are reduced. That is, the GI-V2c and GI-V2t scales are recommended to be utilized for future research as these scales do not incorporate an item with substantial missing data and the performance of these scales were equivalent with the scales that included the DHS Historical Suicide item (GI-V1c and GI-V1t scales).

There is a substantial lack of research that explores the predictive validity of genderinformed factors and their utilization in gender-neutral assessments. It would be beneficial for replication of this study to determine the extent of the generalizability of the findings and whether findings are consistent in women offender populations. Future research should incorporate male comparison samples as well, to help address the current limitations in this study and other studies that incorporate a gender-informed perspective in women offenders. Future 
research should also incorporate items that coincide with the gender-informed poverty and selfefficacy/self-esteem domain to include these in analysis.

Not only is replication needed, but more research that focuses on Indigenous women offenders is also imperative. To date, there is a dearth of research that explores the utilization of gender-informed perspectives with Indigenous women offenders. In fact, future research can explore if there is any differences in which gender-informed predictors are predictive for Indigenous women offenders in comparison to non-Indigenous women offenders. Future research can explore the creation of gender-informed scales pertaining to Indigenous women offenders only, and determine if these gender-informed scales contribute to the incremental predictive validity of the CRS or other gender-neutral assessments. Further, future research should also consider culturally appropriate and relevant factors for Indigenous women that may be potential predictors of institutional misconducts. For instance, researchers can examine experiences of racism within and outside the correctional institution, loss of identity/culture, traumas relating to the residential school experience, and generational impacts of colonialism. Better representation of Indigenous perspectives are needed in research with Indigenous offenders to address culturally sensitive issues and concerns pertinent to Indigenous offenders.

\section{Conclusion}

There is very limited research that explores the inclusion of gender-informed factors to gender-neutral assessments, and to the author's knowledge, this is the first study that examined addition of gender-informed predictors to the CRS. Further, this study was also theoretically driven with a women-centric lens to guide analysis. The current study explored which items in the gender-informed domains would be predictive of any misconducts in women offenders. The items that were predictive of any misconducts were examined if the addition of these items 
produced incremental predictive validity above and beyond the CRS. Overall, results provided support for the predictive accuracy of gender-informed domains to any misconducts and the gender-informed scales produced incremental predictive validity to the CRS. Caution should be given to Indigenous offenders, as results for the incremental predictive validity were mixed. Taken collectively, the area of gender-informed domains as predictive factors to institutional misconduct in women offenders is substantially understudied. More research is needed to verify these findings and if the addition of gender-informed factors to other genderneutral assessments is warranted; especially with Indigenous women offenders. This study provides an adequate foundation for the continuation of future research of gender-informed predictors in women offenders. 


\section{References}

Ægisdóttir, S., White, M. J., Spengler, P. M., Maugherman, A. S., Anderson, L. A., Cook, R. S., . . Rush, J. D. (2006). The meta-analysis of clinical judgment project: Fifty-six years of accumulated research on clinical versus statistical prediction. The Counseling Psychologist, 34(3), 341-382. doi: 10.1177/0011000005285875

American Psychiatric Association. (2000). Diagnostic and statistical manual of mental disorders: DSM-IV-TR. Washington, DC.

Ames, D. R., \& Flynn, F. J. (2007). What breaks a leader: The curvilinear relation between assertiveness and leadership. Journal of Personality and Social Psychology, 92(2), 307324. doi:10.1037/0022-3514.92.2.307

Andrews, D.A., \& Bonta, J. (1995). The level of service inventory-revised. Toronto, Canada: Multihealth Systems.

Andrews, D. A., Guzzo, L., Raynor, P., Rowe, R. C., Rettinger, L. J., Brews, A., \& Wormith, J. S. (2012). Are the major risk/need factors predictive of both female and male reoffending?: A test with the eight domains of the level of service/case management inventory. International Journal of Offender Therapy and Comparative Criminology, 56(1), 113-133. doi:10.1177/0306624X10395716

Anumba, N., Dematteo, D., \& Heilbrun, K. (2012). Social functioning, victimization, and mental health among female offenders. Criminal Justice and Behavior, 39(9), 1204-1218. doi:10.1177/0093854812443489

Arbach-Lucioni, K., Martinez-García, M., \& Andrés-Pueyo, A. (2012). Risk factors for violent behavior in prison inmates: A cross-cultural contribution. Criminal Justice and Behavior, 39(9), 1219-1239. doi:10.1177/0093854812445875 
Archambault, K., Stewart, L., Wilton, G., \& Cousineau, C. (2010). Initial results of the Computerized Mental Health Intake Screening System (CoMHISS) for Federally Sentenced Women. Research Report R-230. Ottawa, ON: Correctional Service Canada.

Asscher, J. J., van der Put, C. E., \& Stams, G. J. J. M. (2015). Gender differences in the impact of abuse and neglect victimization on adolescent offending behavior. Journal of Family Violence, 30(2), 215-225. doi:10.1007/s10896-014- 9668-4

Auditor General of Canada. (2017). Preparing women offenders for release - Correctional service of canada. Retrieved from http://www.oagbvg.gc.ca/internet/English/parl_oag_201711_05_e_42670.html

Austin, J., \& Hardyman, P. L. (2004). Objective prison classification: A guide for correctional agencies (Accession Number 019319). Washington, DC: National Institute of Corrections.

Barnum, G. \& Gobeil, R. (2012). Revalidation of the custody rating scale for Aboriginal and non-Aboriginal women offenders. Research Report R-273. Ottawa, ON: Correctional Service of Canada.

Barrett, M. R., Allenby, K., \& Taylor, K. (2010). Twenty years later: Revisiting The Task Force on Federally Sentenced Women. Research Report R-222. Ottawa: Correctional Service Canada. Retrieved from http://www.csc-scc.gc.ca/005/008/092/005008-0222-01-eng.pdf

Becker, S. P., Kerig, P. K., Lim, J., \& Ezechukwu, R. N. (2012). Predictors of recidivism among delinquent youth: Interrelations among ethnicity, gender, age, mental health problems, and posttraumatic stress. Journal of Child \& Adolescent Trauma, 5(2), 145-160.

doi:10.1080/19361521.2012.671798 
Bengtson, S., \& LÅngström, N. (2007). Unguided clinical and actuarial assessment of reoffending risk: A direct comparison with sex offenders in denmark. Sexual Abuse: A Journal of Research and Treatment, 19(2), 135-153. doi: 10.1177/107906320701900205

Benning, C. L., \& Lahm, K. F. (2016). Effects of Parent-Child relationships on inmate behavior: A comparison of male and female inmates. International Journal of Offender Therapy and Comparative Criminology, 60(2), 189-207. doi:10.1177/0306624X14551402

Blackburn, A. G., \& Trulson, C. R. (2010). Sugar and spice and everything nice? exploring institutional misconduct among serious and violent female delinquents. Journal of Criminal Justice, 38(6), 1132-1140. doi: 10.1016/j.jcrimjus.2010.09.001

Blanchette, K. (2002) 'Classifying female offenders for effective intervention: application of the case-based principles of risk and need', Forum on Correctional Research, 14, 31-5.

Blanchette, K. (2004). III. revisiting effective classification strategies for women offenders in canada. Feminism \& Psychology, 14(2), 231-236. doi:10.1177/0959353504042176

Blanchette, K. (2005). Field-test of a gender-informed security re-classification scale for female offenders. Unpublished thesis dissertation.

Blanchette, K., \& Brown, S.L. (2006). The assessment and treatment of women offenders: An integrative perspective. Hoboken, NJ; Chichester, West Sussex, England: J. Wiley \& Sons.

Blanchette, K., \& Taylor, K. N. (2007). Development and field test of a gender-informed security reclassification scale for female offenders. Criminal Justice and Behavior, 34(3), 362-379. doi:10.1177/0093854806290162

Blanchette, K., Verbrugge, P., \& Wichmann, C. (2002). The custody rating scale, initial security placement, and women offenders. Research Report R-127. Ottawa, ON: Correctional Service of Canada. 
Bloom, B., Owen, B., \& Covington, S. S. (2003). Gender responsive strategies: Theory, policy, guiding principles and practices. In R. Immarigeon (Ed.), Women and girls in the criminal justice system (pp. 29-1-29-21). Kingston, NJ: Civic Research Institute. Retrieved from https://s3.amazonaws.com/static.nicic.gov/Library/018017.pdf

Bonta, J., \& Andrews, D. A. (2017). The psychology of criminal conduct (Sixth ed.). London; NY; Routledge, Taylor and Francis Group.

Bonta, J., Pang, B., \& Wallace-Capretta, S. (1995). Predictors of recidivism among incarcerated female offenders. Prison Journal, 75(3), 277-294. doi:10.1177/0032855595075003002

Bowles, M. A., DeHart, D., \& Webb, J. R. (2012). Family influences on female offenders' substance use: The role of adverse childhood events among incarcerated women. Journal of Family Violence, 27(7), 681-686. doi:10.1007/s10896-012-9450-4

Brennan, T., Austin, J., \& National Institute of Corrections (U.S.). (1997). Women in jail: Classification issues.

Brown, S.L. (2017a). A review of the women offender risk/need research: In search of genderneutral, women-salient, and women specific risk factors. (Report No. R-386). Ottawa: Correctional Service of Canada.

Brown, S. L., \& Motiuk, L.L. (2005). The dynamic factors identification and analysis (DFIA) component of the offender intake (OIA) assessment process: a meta-analytic, psychometric and consultative review. Research Report R-164. Ottawa, ON: Correctional Service of Canada. Retrieved from http://www.csc-scc.gc.ca/research/r164-eng.shtml\#intro

Bui, H. N., \& Morash, M. (2010). The impact of network relationships, prison experiences, and internal transformation on women's success after prison release. Journal of Offender Rehabilitation, 49(1), 1-22. doi: 10.1080/10509670903435381 
Casey-Acevedo, K., Bakken, T., \& Karle, A. (2004). Children visiting mothers in prison: The effects on mothers behaviour and disciplinary adjustment. Australian and New Zealand Journal of Criminology, 37(3), 418-430. doi:10.1375/0004865042194412

Celinska, K., \& Sung, H. (2014). Gender differences in the determinants of prison rule violations. The Prison Journal, 94(2), 220-241. doi:10.1177/0032885514524882

Cesaroni, C., Grol, C., \& Fredericks, K. (2018). Overrepresentation of indigenous youth in Canada's criminal justice system: Perspectives of indigenous young people. Australian and New Zealand Journal of Criminology, , 486581877874. doi:10.1177/0004865818778746

Chesney-Lind, M., Morash, M., \& Stevens, T. (2008). Girls' troubles, girls' delinquency, and gender responsive programming: A review. Australian and New Zealand Journal of Criminology, the, 41(1), 162-189. doi:10.1375/acri.41.1.162

Cihan, A., Davidson, M., \& Sorensen, J. (2017). Analyzing the heterogeneous nature of inmate behavior: Trajectories of prison misconduct. The Prison Journal, 97(4), 431-450. doi: $10.1177 / 0032885517711420$

Cobbina, J. E., Huebner, B. M., \& Berg, M. T. (2012). Men, women, and postrelease offending: An examination of the nature of the link between relational ties and recidivism. Crime \& Delinquency, 58(3), 331-361. doi: 10.1177/0011128710382348

Collica, K. (2010). Surviving incarceration: Two prison-based peer programs build communities of support for female offenders. Deviant Behavior, 31(4), 314-347. doi: $10.1080 / 01639620903004812$

Correctional Service of Canada. (2018a). Security classification and penitentiary placement. Retrieved from: http://www.csc-scc.gc.ca/acts-and-regulations/705-7-cd-eng.shtml\#s8 
Correctional Service of Canada. (2018b). Section 84 releases: Impact on post-release outcomes (Research Report RIB-17-5). Ottawa, ON: Correctional Service of Canada.

Correctional Service of Canada. (2018c). Review of inmate security classification. Retrieved from http://www.csc-scc.gc.ca/lois-et-reglements/710-6-cd-eng.shtml

Correctional Service of Canada. (2017). Women offenders. Retrieved from: http://www.cscscc.gc.ca/publications/092/005007-3012-eng.pdf

Correctional Service of Canada. (2017). Indigenous corrections. Retrieved from http://www.cscscc.gc.ca/publications/005007-3001-eng.shtml

Correctional Service of Canada. (2016a). 2016-2017 Report on Plans and Priorities. Retrieved from: http://www.csc-scc.gc.ca/publications/005007-2604-eng.shtml

Correctional Service of Canada. (2016b). Serving Time. Retrieved from: http://www.cscscc.gc.ca/correctional-process/002001-1000-eng.shtml

Correctional Service of Canada. (2015). Discipline of Inmates. Retrieved from http://www.cscscc.gc.ca/lois-et-reglements/580-cd-eng.shtml

Correctional Service of Canada. (2013a). Mission, values, and ethics framework of correctional service of canada. Retrieved from http://www.csc-scc.gc.ca/acts-and-regulations/001-cdeng.shtml

Correctional Service of Canada. (2013b). History of women's corrections. Retrieved from http://www.csc-scc.gc.ca/women/002002-0003-eng.shtml

Correctional Service of Canada. (2013c). Women's facilities. Retrieved from http://www.cscscc.gc.ca/women/002002-0002-eng.shtml 
Correctional Service of Canada. (2008). Section 2: The mandate, mission, and priorities of correctional service of canada. Retrieved from http://www.csc-scc.gc.ca/text/pblct/sbgo/02-eng.shtml

Correctional Service of Canada. (2007). Computerized mental health intake screening system (comhiss). Retrieved from http://www.csc-scc.gc.ca/atip/007006-0010-eng.shtml Corrections and Conditional Release Act [CCRA]. (1992). Retrieved from http://lawslois.justice.gc.ca/eng/acts/C-44.6/

Daly, K. (1992). Women's pathways to felony court: Feminist theories of lawbreaking and problems of representation. Southern California Review of Law and Women's Studies, 2, $11-52$.

Davidson, M., Sorensen, J., \& Reidy, T. (2016). Gender-responsiveness in corrections: Estimating female inmate misconduct risk using the personality assessment inventory (PAI). Law and Human Behavior, 40(1), 72-81. doi: 10.1037/lhb0000157

DeHart, D., Lynch, S., Belknap, J., Dass-Brailsford, P., \& Green, B. (2014). Life history models of female offending: The roles of serious mental illness and trauma in women's pathways to jail. Psychology of Women Quarterly, 38(1), 138- 151. doi:10.1177/0361684313494357

Derkzen, D., Booth, L., Taylor, K., \& McConnell, A. (2013). Mental health needs of federal female offenders. Psychological Services, 10(1), 24-36. doi:10.1037/a0029653

Derogatis, L.R. (1993). BSI Brief Symptom Inventory. Administration, Scoring, and Procedures Manual (4th Ed.). Minneapolis, MN: National Computer Systems.

Drapalski, A. L., Youman, K., Stuewig, J., \& Tangney, J. (2009). Gender differences in jail inmates' symptoms of mental illness, treatment history and treatment seeking. Criminal Behaviour and Mental Health, 19(3), 193-206. doi:10.1002/cbm.733 
Drury, A. J., \& Delisi, M. (2010). Past is the prologue: Prior adjustment to prison and institutional misconduct. The Prison Journal, 90, 331-352.

Edens, J. F., Poythress, N. G., Lilienfeld, S. O., Patrick, C. J., \& Test, A. (2008). Further evidence of the divergent correlates of the psychopathic personality inventory factors: Prediction of institutional misconduct among male prisoners. Psychological Assessment, 20(1), 86-91. doi: 10.1037/1040-3590.20.1.86

Ewert v. Canada, 2015 FC 1093.

Farr, K. A. (2000). Classification for female inmates: Moving forward. Crime \& Delinquency, 46(1), 3-17. doi: 10.1177/0011128700046001001

Farrell MacDonald, S., Gobeil, R., Biro, S. M., Ritchie, M. B., \& Curno, J. (2015). Women offenders, substance use, and behaviour (Research Report R-358). Ottawa, Ontario: Correctional Service of Canada.

Fazel, S., \& Seewald, K. (2012). Severe mental illness in 33588 prisoners worldwide: Systematic review and metaregression analysis. British Journal of Psychiatry, 200(5), 364373. doi: 10.1192/bjp.bp.111.096370

Ferraro, K. J., \& Moe, A. M. (2003). Mothering, crime, and incarceration. Journal of Contemporary Ethnography, 32(1), 9-40. doi:10.1177/0891241602238937

Gendreau, P., Goggin, C. E., \& Law, M. A. (1997). Predicting prison misconducts. Criminal Justice and Behavior, 24(4), 414-431. doi:10.1177/0093854897024004002

Gehring, K. S. (2016). A direct test of pathways theory. Feminist Criminology, doi: $10.1177 / 1557085116646195$

Gobeil, R. (2008). Assessing security reclassification with male aboriginal and non-aboriginal offenders. Ottawa, ON: Research Branch, Correctional Service of Canada. 
Gobeil, R., \& Blanchette, K. (2007). Revalidation of a gender-informed security reclassification scale for women inmates. Journal of Contemporary Criminal Justice, 23(4), 296-309. doi: $10.1177 / 1043986207309411$

Gobeil, R., Blanchette, K., \& Stewart, L. (2016). A meta-analytic review of correctional interventions for women offenders: Gender-neutral versus gender-informed approaches. Criminal Justice and Behavior, 43(3), 301-322. doi:10.1177/0093854815621100

Gover, A. R., Pérez, D. M., \& Jennings, W. G. (2008). Gender differences in factors contributing to institutional misconduct. The Prison Journal, 88(3), 378-403. doi: $10.1177 / 0032885508322453$

Grant, B., Varis, D., \& Lefebvre, D. (2005). Intensive support units (ISU) for federal offenders with substance abuse problems: An impact analysis. Research Report R-151. Ottawa, ON: Correctional Service of Canada. Retrieved from http://www.csc-scc.gc.ca/research/r151eng.shtml\#institutional_charges

Grant, B., \& Luciani, F. (1998). Security classification using the custody rating scale. Research Report R-67. Ottawa, ON: Correctional Service of Canada.

Green, S. B., \& Salkind, N.J. (2008). Using SPSS for windows and macintosh; Analyzing and understanding data (5th ed.). Upper Saddle River, NJ: Pearson.

Grella, C. E., Lovinger, K., \& Warda, U. S. (2013). Relationships among trauma exposure, familial characteristics, and PTSD: A case-control study of women in prison and in the general population. Women and Criminal Justice, 23(1), 63- 79.

doi:10.1080/08974454.2013.743376

Grieves, A. (2010). An empirical examination of gender neutral, salient, and specific risk factors for male and female criminal recidivism (Order No. MR71710). Available from 
Dissertations \& Theses @ Carleton University; ProQuest Dissertations \& Theses Global. (864801564). Retrieved from http://proxy.library.carleton.ca/login?url=https://searchproquest-com.proxy.library.carleton.ca/docview/864801564?accountid=9894

Hanley, J. A., \& McNeil, B. J. (1983). A method of comparing the areas under receiver operating characteristic curves derived from the same cases. Radiology, 148(3), 839-843.

Hanson, R. K. (2009). The psychological assessment of risk for crime and violence. Canadian Psychology/Psychologie Canadienne, 50(3), 172-182. doi: 10.1037/a0015726

Hare, R. D. (2003). Manual for the Hare Psychopathy Checklist-Revised (2nd ed.). Toronto, ON: Multi-Health Systems.

Harris, P. M. (2006). What community supervision officers need to know about actuarial risk assessment and clinical judgment. Federal Probation, 70(2), 8.

Harris, G. T., Rice, M. E., Quinsey, V. L., \& Cormier, C. A. (2015). Final conclusions. (3rd ed., pp. 267-284). Washington, DC; American Psychological Association. doi: 10.1037/14572010

Hayes, M. (2015). The life pattern of incarcerated women: The complex and interwoven lives of trauma, mental illness, and substance abuse. Journal of Forensic Nursing, 11(4), 214-222. doi:10.1097/JFN.0000000000000092

Herbst, J., Branscomb-Burgess, O., Gelaude, D., Seth, P., Parker, S., \& Fogel, C. (2016). risk profiles of women experiencing initial and repeat incarcerations: Implications for prevention programs. Aids Education and Prevention, 28(4), 299-311. doi:10.1521/aeap.2016.28.4.299 
Holtfreter, K., Reisig, M. D., \& Morash, M. (2004). poverty, state capital, and recidivism among women offenders. Criminology \& Public Policy, 3(2), 185-208. doi:10.1111/j.17459133.2004.tb00035.x

Houck, K., \& Loper, A. (2002). The relationship of parenting stress to adjustment among mothers in prison. American Journal of Orthopsychiatry, 72(4), 548-558. doi:10.1037//0002-9432.72.4.548

Hubbard, D. J., \& Matthews, B. (2008). Reconciling the differences between the "genderresponsive" and the "what works" literatures to improve services for girls. Crime and Delinquency, 54(2), 225-258. doi:10.1177/0011128706296733

Ireland, J. L. (2002). How does assertiveness relate to bullying behaviour among prisoners? Legal and Criminological Psychology, 7(1), 87-100. doi:10.1348/135532502168405

Irwin, J., \& Cressey, D. R. (1962). Thieves, convicts and the inmate culture. Social Problems, 10(2), 142-155. doi: 10.1525/sp.1962.10.2.03a00040

Jordan, J. V. (2008). Recent developments in relational-cultural theory. Women \& Therapy, 31(2-4), 1-4. doi: 10.1080/02703140802145540

Kartsonaki, C. (2016). Survival analysis. Diagnostic Histopathology, 22(7), 263-270. doi:10.1016/j.mpdhp.2016.06.005

Katsiyannis, A., Whitford, D. K., Zhang, D., \& Gage, N. A. (2017). Adult recidivism in united states: A meta-analysis 1994-2015. Journal of Child and Family Studies, , 1-11. doi:10.1007/s10826-017-0945-8

Kennedy, T. D. (1986). Trends in inmate classification: A status report of two computerized psychometric approaches. Criminal Justice and Behavior, 13(2), 165-184. doi: $10.1177 / 0093854886013002003$ 
Kessler, R.C., Adler, L., Barkley, R., Biederman, J., Conners, C.K., Demler, O., \& Zaslavsky, A.M. (2006). The prevalence and correlates of adult adhd in the united states: Results from the national comorbidity survey replication. American Journal of Psychiatry, 163(4), 716723.

Kolivoski, K. M., \& Shook, J. J. (2016). Incarcerating juveniles in adult prisons: Examining the relationship between age and prison behavior in transferred juveniles. Criminal Justice and Behavior, 43(9), 1242-1259. doi: 10.1177/0093854816631793

Kruttschnitt, C. (2016). The politics, and place, of gender in research on crime: The politics, and place, of gender. Criminology, 54(1), 8-29. doi:10.1111/1745-9125.12096

Kuanliang, A., \& Sorensen, J. (2008). Predictors of self-reported prison misconduct. Criminal Justice Studies, 21(1), 27- 35. doi:10.1080/14786010801972662

Kunic, D. (2006). The computerized assessment of substance abuse (casa). Ottawa, ON: Correctional Service of Canada. http://www.cscscc.gc.ca/research/forum/e181/e181e_e.pdf

Kunic, D., \& Grant, B.A. (2006). The computerized assessment of substance abuse (casa): Results from the demonstration project. Ottawa, ON: Correctional Service of Canada. Retrieved from http://www.csc-scc.gc.ca/research/r173-eng.shtml

Kroner, D. G., \& Mills, J. F. (2001). The accuracy of five risk appraisal instruments in predicting institutional misconduct and new convictions. Criminal Justice and Behavior, 28(4), 471 489. doi:10.1177/009385480102800405

Lahm, K. (2016). Official incidents of inmate-on-inmate misconduct at a women's prison: Using importation and deprivation theories to compare perpetrators to victims. Criminal Justice Studies, 29(3), 214-231. doi: 10.1080/1478601X.2016.1154263 
Lahm, K. F. (2017). Violent and nonviolent misconduct among female inmates: An exploration of competing theories. Victims \& Offenders, 12(2), 175-204. doi: $10.1080 / 15564886.2015 .1017132$

Lombroso, C., \& Ferrero, W. (1895). The female offender. London; Peter Own Limited.

Luciani, F. (1997). Tried and true: Proof that the Custody Rating Scale is still reliable and valid. Forum on Corrections Research, 9, 13-17.

Luciani, F. P., Motiuk, L. L., \& Nafekh, M. (1996). An operational review of the Custody Rating Scale: Reliability, validity, and practical utility (Research Report R-47). Ottawa, ON: CSC.

Luciani, F., Taylor, G., \& Motiuk, L. (1998). National field test results of the security reclassification protocol: Final report. Unpublished Manuscript. Correctional Service of Canada, Ottawa.

MacDonald, S. F., Gobeil, R., Biro, S. M., Ritchie, M. B., \& Curno, J. (2015). Women offenders, substance use, and behaviour (Research Report R-358). Ottawa, Ontario: Correctional Service of Canada.

Martin, M. S., Wamboldt, A. D., O'Connor, S. L., Fortier, J., \& Simpson, A. I. F. (2013). A comparison of scoring models for computerised mental health screening for federal prison inmates. Criminal Behaviour and Mental Health, 23(1), 6- 17. doi:10.1002/cbm.1853

McKeown, A. (2010). Female offenders: Assessment of risk in forensic settings. Aggression and Violent Behavior, 15(6), 422-429. doi: 10.1016/j.avb.2010.07.004

Michael, M.S., Crocker, A.G., Potter, B.K., Wells, G.A., Grace, R.M., \& Colman, I. (2018). Mental health screening differences in access to care among prisoners. The Canadian Jouranl of Psychiatry, 63(10), 692-700. doi: 10.1177/0706743718762099

Miller, J. B. (1976). Toward a new psychology of women. 
Miller, M., \& Mullins, C.W. (2009). Feminist theories of girls' delinquency. In M. Zahn (Eds.), The delinquent girl (pp. 30-49). Philadelphia, PA: Temple University Press

Mills, J. F., \& Kroner, D. G. (2008). Predicting suicidal ideation with the depression hopelessness and suicide screening form (DHS). Journal of Offender Rehabilitation, 47(12), 74-100. doi:10.1080/10509670801940680

Mills, J.F. \& Kroner, D.G. (2004). A new instrument to screen for depression, hopelessness and suicide in offenders. Psychological Services, 1, 83-91.

Motiuk, L. (1997). Classification for correctional programming: The offender intake assessment (OIA) process. Forum on Corrections Research, 9, 18-22.

Naglieri., A., \& Bardosa, N. (1997) General Ability Measure for Adults. Minneapolis, MN: National Computer Systems.

Public Safety Canada. (2017). Corrections and conditional release: Statistical overview. Retrieved from: https://www.publicsafety.gc.ca/cnt/rsrcs/pblctns/ccrso-2016/ccrso-2016en.pdf

Reidy, T., Cihan, A., \& Sorensen, J. (2017). Women in prison: Investigating trajectories of institutional female misconduct. Journal of Criminal Justice, 52, 49-56. doi:10.1016/j.jcrimjus.2017.07.013

Rettinger, L. J., \& Andrews, D. A. (2010). General risk and need, gender specificity, and the recidivism of female offenders. Criminal Justice and Behavior, 37, 29-46.

Rice, M. E., \& Harris, G. T. (2005). Comparing effect sizes in follow-up studies: ROC area, cohen's d, and r. Law and Human Behavior, 29(5), 615-620. doi:10.1007/s10979-005$6832-7$ 
Rice, M. E., \& Harris, G. T. (1995). Violent recidivism: Assessing predictive validity. Journal of Consulting and Clinical Psychology, 63(5), 737-748. doi:10.1037/0022-006X.63.5.737

Rubenfeld S. (2014). An Examination of a Reweighted Custody Rating Scale for Women.

Research Report R289. Ottawa, Ontario: Correctional Service of Canada.

Ruddell, R., \& Gottschall, S. (2011). Are all gangs equal security risks? an investigation of gang types and prison misconduct. American Journal of Criminal Justice, 36(3), 265-279. doi: $10.1007 / \mathrm{s} 12103-011-9108-4$

Salisbury, E. J., \& Van Voorhis, P. (2009). Gendered pathways: A quantitative investigation of women probationers' paths to incarceration. Criminal Justice and Behavior, 36, 541-566. doi: $10.1177 / 0093854809334076$

Salisbury, E. J., Van Voorhis, P., \& Spiropoulos, G. V. (2009). The predictive validity of a gender-responsive needs assessment: An exploratory study. Crime \& Delinquency, 55(4), 550-585. doi:10.1177/0011128707308102

Scott, C. K., Grella, C. E., Dennis, M. L., \& Funk, R. R. (2016). A time-varying model of risk for predicting recidivism among women offenders over 3 years following their release from jail. Criminal Justice and Behavior, 43(9), 1137-1158. doi:10.1177/0093854816632551

Shepherd, S. M., \& Lewis-Fernandez, R. (2016). Forensic risk assessment and cultural diversity: Contemporary challenges and future directions. Psychology, Public Policy, and Law, 22(4), 427-438. doi:10.1037/law0000102

Simons, R., \& Barr, A. (2014). Shifting perspectives: Cognitive changes mediate the impact of romantic relationships on desistance from crime. Justice Quarterly, 31(5), 793-821. doi: $10.1080 / 07418825.2012 .704388$ 
Skinner, H. A. (1982). The Drug Abuse Screening Test. Addictive Behaviors, 7, 363-371.

Skinner, H. A., \& Horn, J. L. (1984). Alcohol Dependence Scale (ADS): Users Guide. Toronto, ON: Addiction Research Foundation.

Solicitor General of Canada (1987). Development of a security classification model for Canadian federal offenders. Ottawa, ON: Author.

Stalans, L. J., \& Lurigio, A. J. (2015). Parenting and intimate relationship effects on women offenders' recidivism and noncompliance with probation. Women \& Criminal Justice, 25(3), 152-168. doi:10.1080/08974454.2014.909764

Steiner, B., Butler, H., \& Ellison, J. (2014). Causes and correlates of prison inmate misconduct: A systematic review of the evidence. Journal of Criminal Justice, 42(6), 462-470. doi:10.1016/j.jcrimjus.2014.08.001

Steiner, B., \& Wooldredge, J. (2009). Individual and environmental effects on assaults and nonviolent rule breaking by women in prison. Journal of Research in Crime and Delinquency, 46(4), 437-467. doi:10.1177/0022427809341936

Steiner, B., \& Wooldredge, J. (2014). Sex differences in the predictors of prisoner misconduct. Criminal Justice and Behavior, 41(4), 433-452. doi:10.1177/0093854813504404

Steward, L.A., Archambault, K., \& Wilton, G. (2013). Results of the computerized mental health screening system for female offenders. Journal of Socialomics, 2(2): 103. doi: $10.4172 / 2167-0358.1000103$

Stewart, S., Wardrop, K., Wilton, G., Thompson, J., Derkzen, D., \& Motiuk, L. (2017). Reliability and validity of the Dynamic Factors Identification and Analysis - Revised (Research Report R-395). Ottawa, Ontario: Correctional Service of Canada. 
Stewart, L.A., Wilton, G. \& Malek, A. (2011). Validation of the Computerised Mental Health Intake Screening System (CoMHISS) in a Federal Male Offender Population. Research Report R 244. Ottawa, ON.: Correctional Service of Canada

Swan, A. C., \& Goodman-Delahunty, J. (2013). The relationship between drug use and crime among police detainees: Does gender matter? International Journal of Forensic Mental Health, 12(2), 107-115. doi:10.1080/14999013.2013.787561

Sykes, G. M. (1958). The society of captives: A study of a maximum security prison. Princeton, N. J: Princeton University Press.

Tasca, M., Griffin, M. L., \& Rodriguez, N. (2010). The effect of importation and deprivation factors on violent misconduct: An examination of black and latino youth in prison. Youth Violence and Juvenile Justice, 8(3), 234-249. doi:10.1177/1541204010366619

Taylor, C. (2015). Recent victimization and recidivism: The potential moderating effects of family support. Violence and Victims, 30(2), 342-360. doi:10.1891/0886-6708.VV-D-1300139

Taylor, A. (2008). Substance use and abuse: Women's criminal reoffending in new zealand. Affilia, 23(2), 167-178. doi:10.1177/0886109908314328

Thompson, J., Forrester, T., \& Stewart, L. (2015). Factors related to community supervision outcomes: Revocations (Research Report R-304). Ottawa, ON: Correctional Service of Canada.

Thomson, N., Towl, G., \& Centifanti, L. (2016). The habitual female offender inside: How psychopathic traits predict chronic prison violence. Law and Human Behavior, 40(3), 257269. doi: $10.1037 / \mathrm{hb} 0000178$ 
van der Put, C. E., \& de Ruiter, C. (2016). Child maltreatment victimization by type in relation to criminal recidivism in juvenile offenders. BMC Psychiatry, 16, 24. doi:10.1186/s12888016-0731-y

Van Voorhis, P., Wright, E., Salisbury, E., \& Bauman, A. (2010). Women's risk factors and their contributions to existing risk/needs assessment: The current status of a gender-responsive supplement. Criminal Justice and Behavior, 37(3), 261-288. doi: $10.1177 / 0093854809357442$

Verona, E., \& Carbonell, J. L. (2000). Female violence and personality: Evidence for a pattern of overcontrolled hostility among one-time violent female offenders. Criminal Justice and Behavior, 27(2), 176-195. doi:10.1177/0093854800027002003

Walters, G. D. (2015). Criminal thinking as a predictor of prison misconduct and mediator of the static Risk-Infractions relationship. The Prison Journal, 95(3), 353-369. doi: $10.1177 / 0032885515587468$

Webster, C. D., Douglas, K. S., Eaves, D., \& Hart, S. D. (1997). HCR-20: Assessing risk for violence (Version 2). Vancouver, British Columbia, Canada: Mental Health, Law, \& Policy Institute, Simon Fraser University

Wesley, M., Canada. Aboriginal Corrections Policy Unit, \& Canada. Public Safety Canada. (2012). Marginalized, the aboriginal women's experience in federal corrections. (No. APC 33 CA 2012). Ottawa: Public Safety Canada.

Wulf-Ludden, T. (2016). Pseudofamilies, misconduct, and the utility of general strain theory in a women's prison. Women \& Criminal Justice, 26(4), 233-259.

doi:10.1080/08974454.2015.1113154 
Wright, E. M., DeHart, D. D., Koons-Witt, B. A., \& Crittenden, C. A. (2013). 'Buffers' against crime? exploring the roles and limitations of positive relationships among women in prison. Punishment \& Society, 15(1), 71-95. doi:10.1177/1462474512466199

Wright, E. M., Salisbury, E. J., \& Van Voorhis, P. (2007). Predicting the prison misconducts of women offenders: The importance of gender-responsive needs. Journal of Contemporary Criminal Justice, 23(4), 310-340. doi:10.1177/1043986207309595

Young, M. E. (2016). The problem with categorical thinking by psychologists. Behavioural Processes, 123, 43-53. doi:10.1016/j.beproc.2015.09.009

Yudko, E., Lozhkina, O., \& Fouts, A. (2007). A comprehensive review of the psychometric properties of the Drug Abuse Screening Test. Journal of Substance Abuse Treatment, 32, 189-198. 


\section{Appendix A}

\section{Custody Rating Scale}

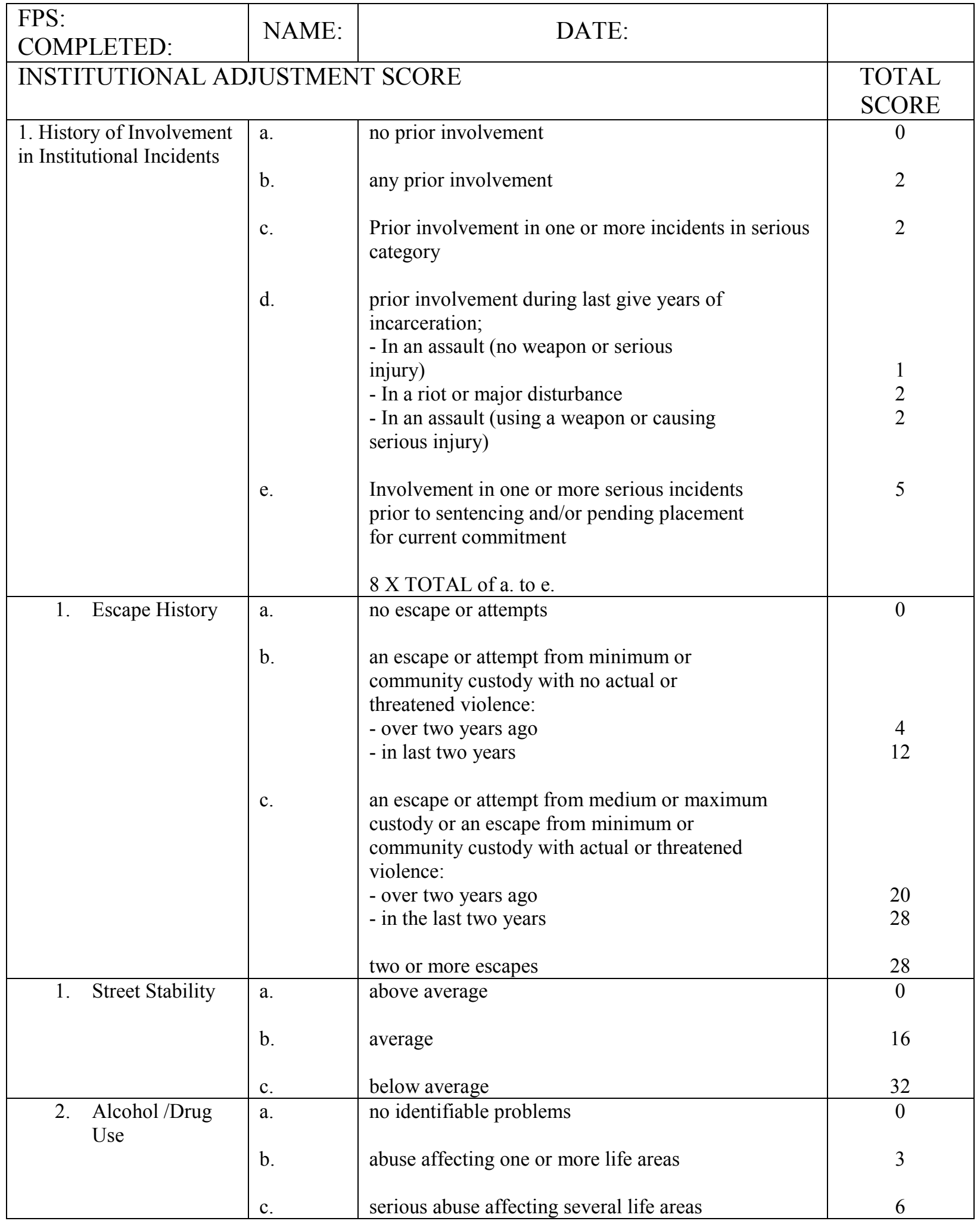




\begin{tabular}{|c|c|c|c|}
\hline $\begin{array}{l}\text { FPS: } \\
\text { COMPLETED: }\end{array}$ & NAME: & DATE: & \\
\hline \multicolumn{3}{|c|}{ INSTITUTIONAL ADJUSTMENT SCORE } & $\begin{array}{l}\text { TOTAL } \\
\text { SCORE }\end{array}$ \\
\hline \multirow{13}{*}{$\begin{array}{l}\text { 1. Age (At any time } \\
\text { of sentencing) }\end{array}$} & a. & 18 years or less & 24 \\
\hline & b. & 19 years & 32 \\
\hline & c. & 20 years & 30 \\
\hline & d. & 21 years & 18 \\
\hline & e. & 22 years & 16 \\
\hline & f. & 23 years & 14 \\
\hline & g. & 24 years & 12 \\
\hline & h. & 25 years & 10 \\
\hline & i. & 26 years & 08 \\
\hline & $\mathrm{j}$. & 27 years & 06 \\
\hline & k. & 28 years & 04 \\
\hline & 1. & 29 years & 02 \\
\hline & $\mathrm{m}$ & 30 years or more & 00 \\
\hline
\end{tabular}




\begin{tabular}{|c|c|c|c|}
\hline \multicolumn{3}{|c|}{ SECURITY RISK SCORE } & \multirow[b]{2}{*}{$\begin{array}{l}\text { TOTAL SCORE } \\
0 \\
3 \\
6 \\
9 \\
12 \\
15 \\
\end{array}$} \\
\hline $\begin{array}{l}\text { 1. Number of prior } \\
\text { convictions }\end{array}$ & $\begin{array}{l}\mathrm{a} \\
\mathrm{b} \\
\mathrm{c} \\
\mathrm{d} \\
\mathrm{e} \\
\mathrm{f}\end{array}$ & $\begin{array}{l}\text { none } \\
\text { one } \\
2 \text { to } 4 \\
5 \text { to } 9 \\
10 \text { to } 14 \\
\text { over } 15 \\
\end{array}$ & \\
\hline $\begin{array}{l}\text { 2. Most serious } \\
\text { outstanding charge }\end{array}$ & $\begin{array}{l}\text { a. } \\
\text { b. } \\
\text { c. } \\
\text { d. } \\
\text { e. }\end{array}$ & $\begin{array}{l}\text { no outstanding charges } \\
\text { minor } \\
\text { moderate } \\
\text { serious } \\
\text { major/extreme }\end{array}$ & $\begin{array}{l}0 \\
12 \\
15 \\
25 \\
35\end{array}$ \\
\hline $\begin{array}{l}\text { 3. Severity of current } \\
\text { offence }\end{array}$ & $\begin{array}{l}\text { a. } \\
\text { b. } \\
\text { c. }\end{array}$ & $\begin{array}{l}\text { minor or moderate } \\
\text { serious or major } \\
\text { extreme }\end{array}$ & $\begin{array}{l}12 \\
36 \\
69\end{array}$ \\
\hline 4. Sentence length & $\begin{array}{l}\text { a. } \\
\text { b. } \\
\text { c. } \\
\text { d. }\end{array}$ & $\begin{array}{l}1 \text { day to } 4 \text { years } \\
5 \text { to } 9 \text { years } \\
10 \text { to } 24 \text { years } \\
\text { over } 24 \text { years }\end{array}$ & $\begin{array}{l}5 \\
20 \\
45 \\
65\end{array}$ \\
\hline 5. Street Stability & $\begin{array}{l}\text { a. } \\
\text { b. } \\
\text { c. } \\
\text { d. }\end{array}$ & $\begin{array}{l}\text { above average } \\
\text { average } \\
\text { below average } \\
\text { Other (i.e., convicted of } \\
\text { criminal organization } \\
\text { offences or terrorism } \\
\text { offences) }\end{array}$ & $\begin{array}{l}0 \\
5 \\
10 \\
20\end{array}$ \\
\hline $\begin{array}{l}\text { 6. Prior parole and/or } \\
\text { statutory release }\end{array}$ & $\begin{array}{l}\text { a. } \\
\text { b. } \\
\text { c. }\end{array}$ & $\begin{array}{l}\text { None } \\
1 \text { point for each prior } \\
\text { parole release } \\
2 \text { points for each prior } \\
\text { statutory release }\end{array}$ & 0 \\
\hline $\begin{array}{l}\text { 7. Age (at time of } \\
\text { admission) }\end{array}$ & $\begin{array}{l}\text { a. } \\
\text { b. } \\
\text { c. } \\
\text { d. } \\
\text { e. } \\
\text { f. }\end{array}$ & $\begin{array}{l}25 \text { years or less } \\
26 \text { years } \\
27 \text { years } \\
28 \text { years } \\
29 \text { years } \\
30 \text { years }\end{array}$ & $\begin{array}{l}30 \\
27 \\
24 \\
21 \\
18 \\
15\end{array}$ \\
\hline
\end{tabular}




\begin{tabular}{|l|l|l|l|}
\hline \multicolumn{2}{|l|}{ SECURITY RISK SCORE } & TOTAL SCORE \\
\hline 7. Age (at time of & g. & 31 years & 12 \\
admission) & h. & 32 years & 09 \\
& i. & 33 years & 06 \\
& j. & 34 years & 03 \\
& k & 35 years or more & 00 \\
\hline
\end{tabular}


Appendix B

Correlation Matrix of Gender-informed Substance Abuse Domain

Table B1

Correlation Matrix of Gender-informed Substance Abuse Domain with Substance Abuse Domain Using N=1528

\begin{tabular}{|c|c|c|c|c|c|c|c|c|c|c|c|c|c|c|c|c|c|c|c|c|}
\hline Measure & 1 & 2 & 3 & 4 & 5 & 6 & 7 & 8 & 9 & 10 & 11 & 12 & 13 & 14 & 15 & 16 & 17 & 18 & 19 & 20 \\
\hline 1. SUB01 & - & & & & & & & & & & & & & & & & & & & \\
\hline 2. SUB02 & $.43 * *$ & - & & & & & & & & & & & & & & & & & & \\
\hline 3. SUB03 & $.55 * *$ & $.50 * *$ & - & & & & & & & & & & & & & & & & & \\
\hline 4. SUB04 & $.40 * *$ & $.69 * *$ & $.47 * *$ & - & & & & & & & & & & & & & & & & \\
\hline 5.SUB05 & $.47 * *$ & $.73 * *$ & $.54 * *$ & $.74 * *$ & - & & & & & & & & & & & & & & & \\
\hline 6. SUB06 & $.47 * *$ & $.73 * *$ & $.52 * *$ & $.72 * *$ & $.87 * *$ & - & & & & & & & & & & & & & & \\
\hline 7. SUB07 & $.43 * *$ & $.82 * *$ & $.51 * *$ & $.73^{* *}$ & $.80 * *$ & $.79 * *$ & - & & & & & & & & & & & & & \\
\hline 8. SUB08 & $.65 * *$ & $.27 * *$ & $.50 * *$ & $.27 * *$ & $.29 * *$ & $.28 * *$ & $.26^{* *}$ & - & & & & & & & & & & & & \\
\hline 9. SUB09 & $.45^{* *}$ & $.29 * *$ & $.51 * *$ & $.28 * *$ & $.26 * *$ & $.25^{* *}$ & $.26^{* *}$ & $.59 * *$ & - & & & & & & & & & & & \\
\hline 10. SUB10 & $.47 * *$ & $.26 * *$ & $.57 * *$ & $.27 * *$ & $.30 * *$ & $.27 * *$ & $.25^{* *}$ & $.58 * *$ & $.75 * *$ & - & & & & & & & & & & \\
\hline 11. SUB11 & $.38 * *$ & $.22 * *$ & $.43 * *$ & $.38^{* *}$ & $.25 * *$ & $.24 * *$ & $.22 * *$ & $.51 * *$ & $.74 * *$ & $.66^{* *}$ & - & & & & & & & & & \\
\hline 12. SUB12 & $.44 * *$ & $.25 * *$ & $.51 * *$ & $.29 * *$ & $.30 * *$ & $.28 * *$ & $.25 * *$ & $.57 * *$ & $.78 * *$ & $.72 * *$ & $.80 * *$ & - & & & & & & & & \\
\hline 13. SUB13 & $.44 * *$ & $.27 * *$ & $.51 * *$ & $.30 * *$ & $.31 * *$ & $.32 * *$ & $.27 * *$ & $.56^{* *}$ & $.77 * *$ & $.72 * *$ & $.78 * *$ & $.90 * *$ & - & & & & & & & \\
\hline
\end{tabular}




\begin{tabular}{|c|c|c|c|c|c|c|c|c|c|c|c|c|c|c|c|c|c|c|c|c|}
\hline Measure & 1 & 2 & 3 & 4 & 5 & 6 & 7 & 8 & 9 & 10 & 11 & 12 & 13 & 14 & 15 & 16 & 17 & 18 & 19 & 20 \\
\hline 14. SUB14 & $.44 * *$ & $.25 * *$ & $.51 * *$ & $.28 * *$ & $.27 * *$ & $.27 * *$ & $.26 * *$ & $.57 * *$ & $.78 * *$ & $.70 * *$ & $.76^{* *}$ & $.81 * *$ & $.82 * *$ & - & & & & & & \\
\hline 15. SUB15 & $.52 * *$ & $.39 * *$ & $.50 * *$ & $.35 * *$ & $.41 * *$ & $.42 * *$ & $.40 * *$ & $.53 * *$ & $.61 * *$ & $.59^{* *}$ & $.58^{* *}$ & $.64 * *$ & $.63 * *$ & $.63 * *$ & & & & & & \\
\hline 16. SUB16 & $.47 * *$ & $.51 * *$ & $.50 * *$ & $.46^{* *}$ & $.52 * *$ & $.53 * *$ & $.52 * *$ & $.41 * *$ & $.43 * *$ & $.40^{* *}$ & $.37 * *$ & $.42 * *$ & $.42 * *$ & $.42 * *$ & $.48 * *$ & - & & & & \\
\hline 17. SUB17 & $.52 * *$ & $.42 * *$ & $.52 * *$ & $.38 * *$ & $.44 * *$ & $.45 * *$ & $.43 * *$ & $.54 * *$ & $.63 * *$ & $.57 * *$ & $.61 * *$ & $.68 * *$ & $.68 * *$ & $.70 * *$ & $.82 * *$ & $.52 * *$ & - & & & \\
\hline 18. SUB18 & $.29 * *$ & $.22 * *$ & $.32 * *$ & $.20 * *$ & $.21 * *$ & $.21 * *$ & $.22 * *$ & $.37 * *$ & $.45 * *$ & $.40 * *$ & $.41 * *$ & $.46^{* *}$ & $.44 * *$ & $.44 * *$ & $.47 * *$ & $.30 * *$ & $.45 * *$ & - & & \\
\hline 19. ADS & $.42 * *$ & $.21 * *$ & $.45^{* *}$ & $.24 * *$ & $.22 * *$ & $.21 * *$ & $.19 * *$ & $.55 * *$ & $.72 * *$ & $.66^{* *}$ & $.71 * *$ & $.75 * *$ & $.72 * *$ & $.72 * *$ & $.59 * *$ & $.39 * *$ & $.63 * *$ & $.47 * *$ & - & \\
\hline 20. DAST & $.46^{* *}$ & $.62 * *$ & $.51 * *$ & $.57 * *$ & $.59 * *$ & $.60^{* *}$ & $.65^{* *}$ & $.33 * *$ & $.29 * *$ & $.32 * *$ & $.27 * *$ & $.27 * *$ & $.29 * *$ & $.28 * *$ & $.42 * *$ & $.47 * *$ & $.44 * *$ & $.25^{* *}$ & $.35 * *$ & - \\
\hline
\end{tabular}




\section{Appendix C}

Pearson Product Moment Correlations of the Gender-informed Substance Abuse Domain

Table C1

Pearson Product Moment Correlations of Gender-informed Substance Abuse Domain with Mental Health Domain Using N=1528

\begin{tabular}{|c|c|c|c|c|c|c|}
\hline Measure & $\begin{array}{l}\text { GSI Subscale of the } \\
\text { Brief Symptom } \\
\text { Inventory }\end{array}$ & DHS Depression & $\begin{array}{l}\text { DHS Current } \\
\text { Suicidal } \\
\text { Ideation }\end{array}$ & $\begin{array}{c}\text { DHS } \\
\text { Hopelessness }\end{array}$ & $\begin{array}{l}\text { DHS Cognitive } \\
\text { Indicator }\end{array}$ & $\begin{array}{l}\text { DHS Historical } \\
\text { Suicide }\end{array}$ \\
\hline SUB01 & $.15^{* *}$ & $.16^{* *}$ & $.07 *$ & $.08 * *$ & .02 & $.20 * *$ \\
\hline SUB02 & $.16^{* *}$ & $.13^{* *}$ & $.08 * *$ & $.12 * *$ & .05 & $.20 * *$ \\
\hline SUB03 & $.18^{* *}$ & $.17 * *$ & $.07 *$ & $.09 * *$ & $.07 *$ & $.27 * *$ \\
\hline SUB04 & $.11 * *$ & $.09 * *$ & $.06^{*}$ & $.07 *$ & .03 & $.18 * *$ \\
\hline SUB05 & $.17 * *$ & $.14^{* *}$ & $.08^{*}$ & $.09 * *$ & .04 & $.21 * *$ \\
\hline SUB06 & $.14^{* *}$ & $.11 * *$ & $.06^{*}$ & $.08^{*}$ & .03 & $.21 * *$ \\
\hline SUB07 & $.13 * *$ & $.11^{* *}$ & $.07 *$ & $.08 * *$ & .04 & $.21 * *$ \\
\hline SUB08 & $.14 * *$ & $.19^{* *}$ & .05 & $.08 * *$ & .03 & $.18 * *$ \\
\hline SUB09 & $.23 * *$ & $.26^{* *}$ & .06 & $.13^{* *}$ & $.08 * *$ & $.22 * *$ \\
\hline SUB10 & $.23 * *$ & $.25^{* *}$ & $.08 * *$ & $.13 * *$ & $.08^{*}$ & $.23 * *$ \\
\hline \multirow[t]{2}{*}{ SUB11 } & $.20 * *$ & $.24 * *$ & $.07^{*}$ & $.15^{* *}$ & .05 & $.18^{* *}$ \\
\hline & & & & & & (Conti \\
\hline
\end{tabular}




\begin{tabular}{|c|c|c|c|c|c|c|}
\hline Measure & $\begin{array}{l}\text { GSI Subscale of the } \\
\text { Brief Symptom } \\
\text { Inventory }\end{array}$ & DHS Depression & $\begin{array}{c}\text { DHS Current } \\
\text { Suicidal } \\
\text { Ideation }\end{array}$ & $\begin{array}{c}\text { DHS } \\
\text { Hopelessness }\end{array}$ & $\begin{array}{l}\text { DHS Cognitive } \\
\text { Indicator }\end{array}$ & $\begin{array}{l}\text { DHS Historical } \\
\text { Suicide }\end{array}$ \\
\hline SUB12 & $.23 * *$ & $.27 * *$ & $.08^{*}$ & $.14 * *$ & $.06^{*}$ & $.22 * *$ \\
\hline SUB13 & $.23^{* *}$ & $.26^{* *}$ & .06 & $.14^{* *}$ & $.06^{*}$ & $.21 * *$ \\
\hline SUB14 & $.20 * *$ & $.23^{* *}$ & $.06^{*}$ & $.13^{* *}$ & $.07 *$ & $.18^{* *}$ \\
\hline SUB15 & $.22 * *$ & $.24 * *$ & .03 & $.10^{* *}$ & .01 & $.17 * *$ \\
\hline SUB16 & $.14^{* *}$ & $.17^{* *}$ & $.09 * *$ & $.12 * *$ & .06 & $.26^{* *}$ \\
\hline SUB17 & $.22 * *$ & $.23 * *$ & .05 & $.13^{* *}$ & .05 & $.20 * *$ \\
\hline SUB18 & $.17 * *$ & $.19 * *$ & $.09 * *$ & $.13^{* *}$ & $.09 * *$ & $.18 * *$ \\
\hline ADS & $.27 * *$ & $.23 * *$ & $.12 * *$ & $.19 * *$ & $.09 * *$ & $.30 * *$ \\
\hline DAST & $.27 * *$ & $.31 * *$ & $.07 *$ & $.18^{*}$ & $.09 * *$ & $.24 * *$ \\
\hline
\end{tabular}

Note: $* p<.05 . * * p<.01 . * * * p<.001$ 


\section{Table C2}

Pearson Product Moment Correlations of the Gender-informed Substance Abuse Domain with Relationship Dysfunction Domain Using $N=1528$

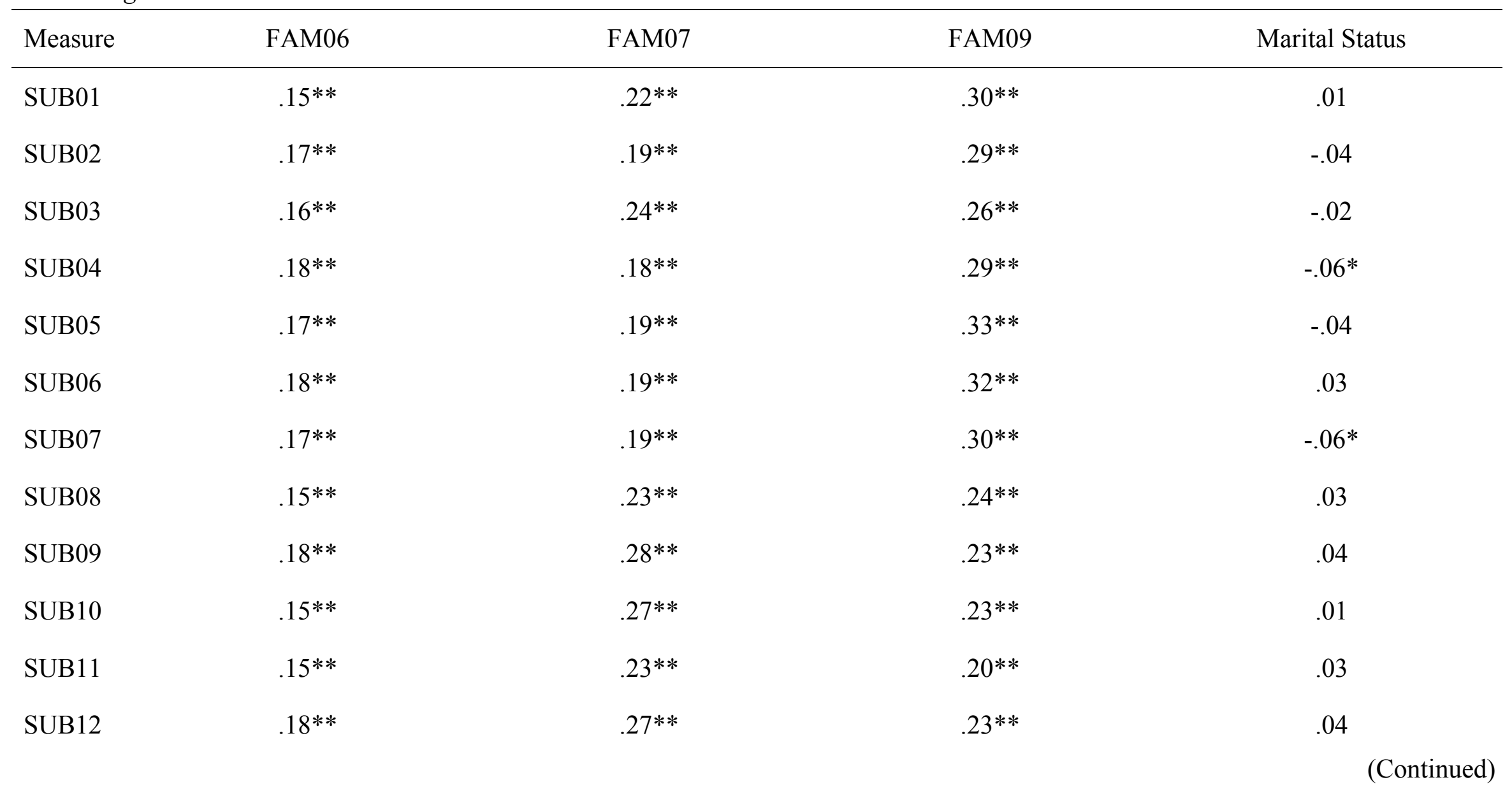




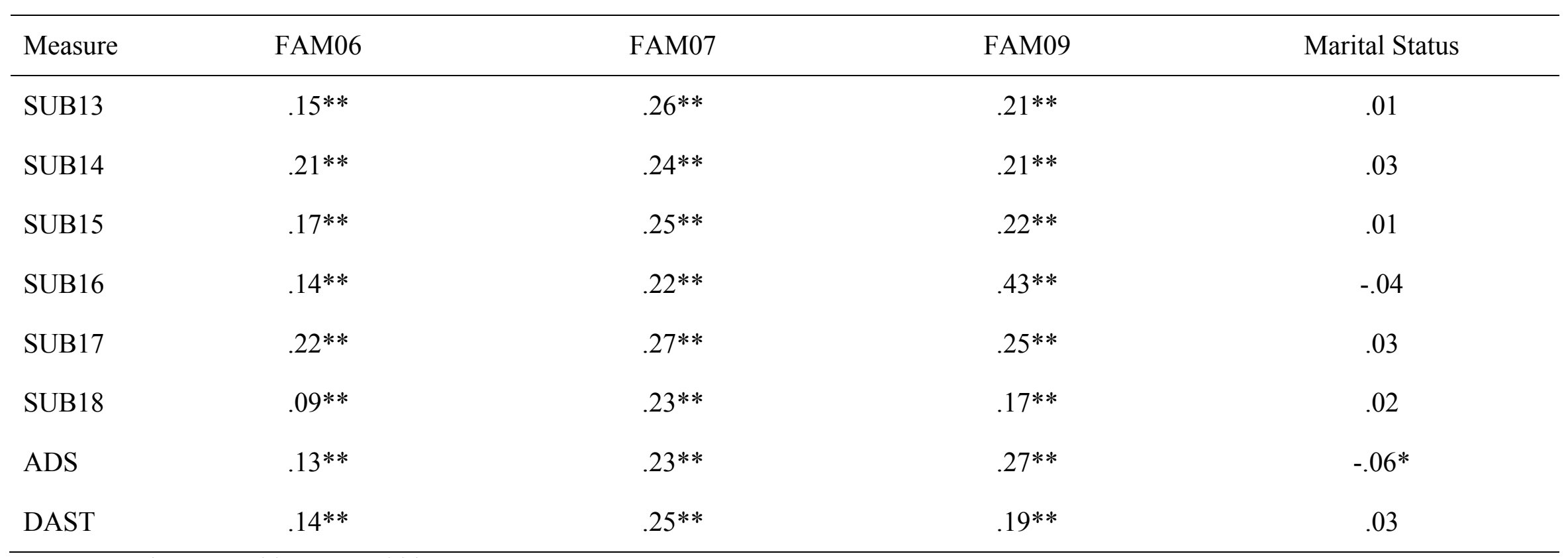

Note: ${ }^{*} p<.05 .{ }^{* *} p<.01 .{ }^{* * *} p<.001$ 
Table C3

Pearson Product Moment Correlations of the Gender-informed Substance Abuse Domain with Parental/Family Issues Domain Using $N=1528$

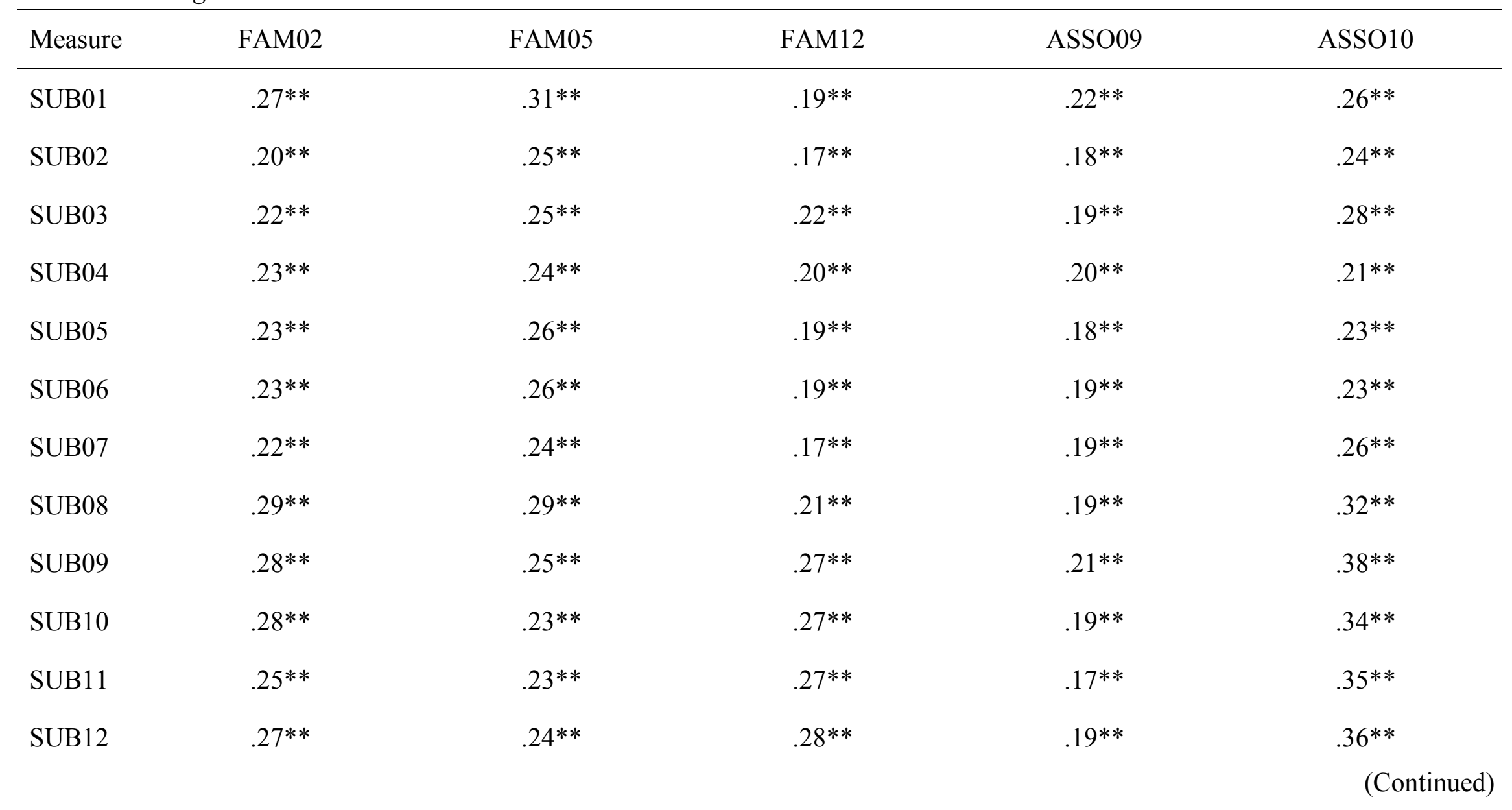




\begin{tabular}{|c|c|c|c|c|c|}
\hline SUB13 & $.28 * *$ & $.25^{* *}$ & $.26^{* *}$ & $.20 * *$ & $.37 * *$ \\
\hline SUB15 & $.26^{* *}$ & $.27 * *$ & $.24 * *$ & $.18 * *$ & $.36 * *$ \\
\hline SUB17 & $.25^{* *}$ & $.26^{* *}$ & $.25^{* *}$ & $.20 * *$ & $.34 * *$ \\
\hline SUB18 & $.19 * *$ & $.18^{* *}$ & $.20 * *$ & $.10 * *$ & $.22 * *$ \\
\hline ADS & $.22 * *$ & $.23 * *$ & $.17^{* *}$ & $.18 * *$ & $.27 * *$ \\
\hline
\end{tabular}

Note: $* p<.05 . * * p<.01 . * * * p<.001$. 


\section{Table C4}

Pearson Product Moment Correlations of the Gender-informed Substance Abuse Domain with Victimization Domain Using N=1528

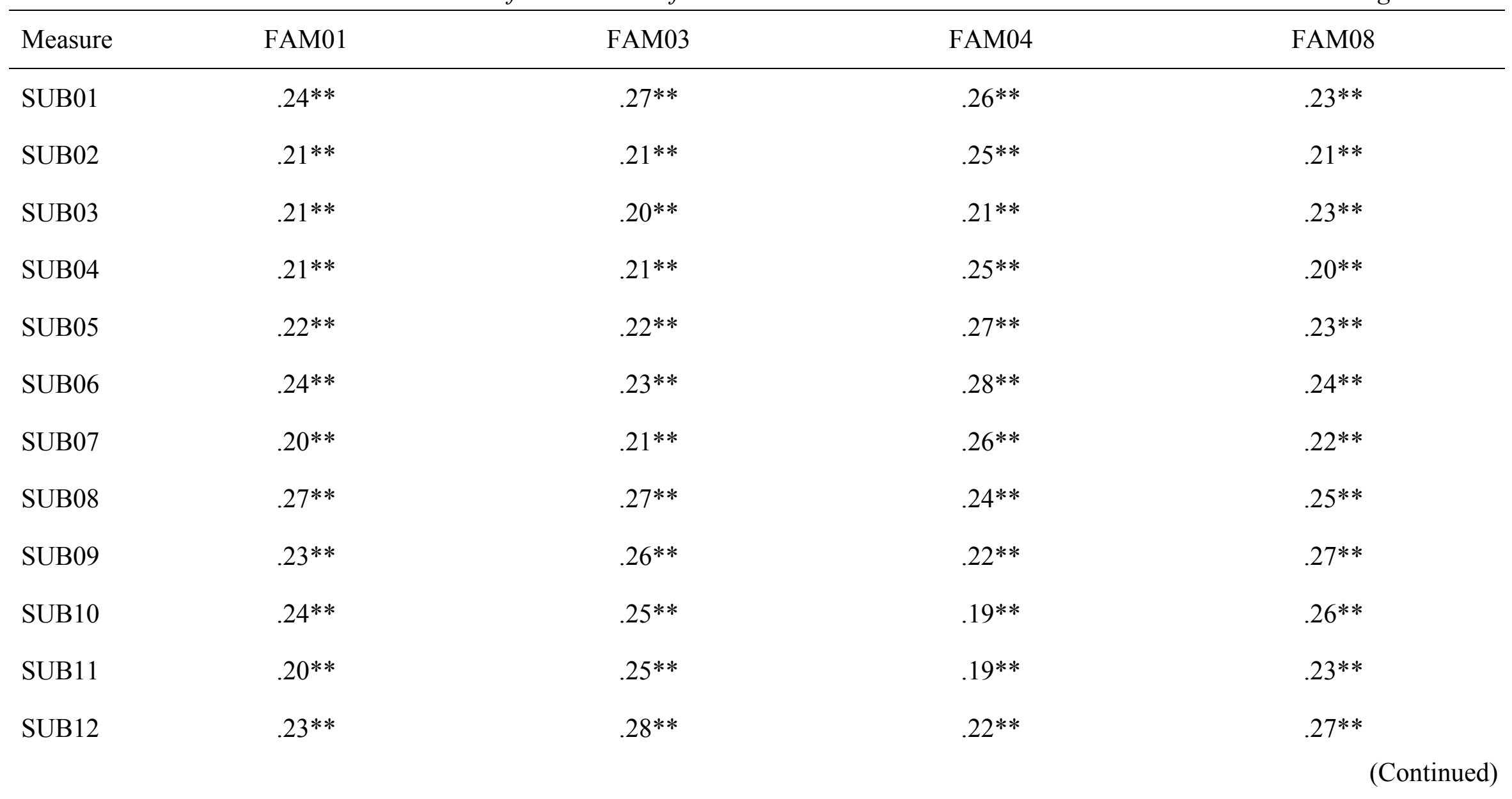




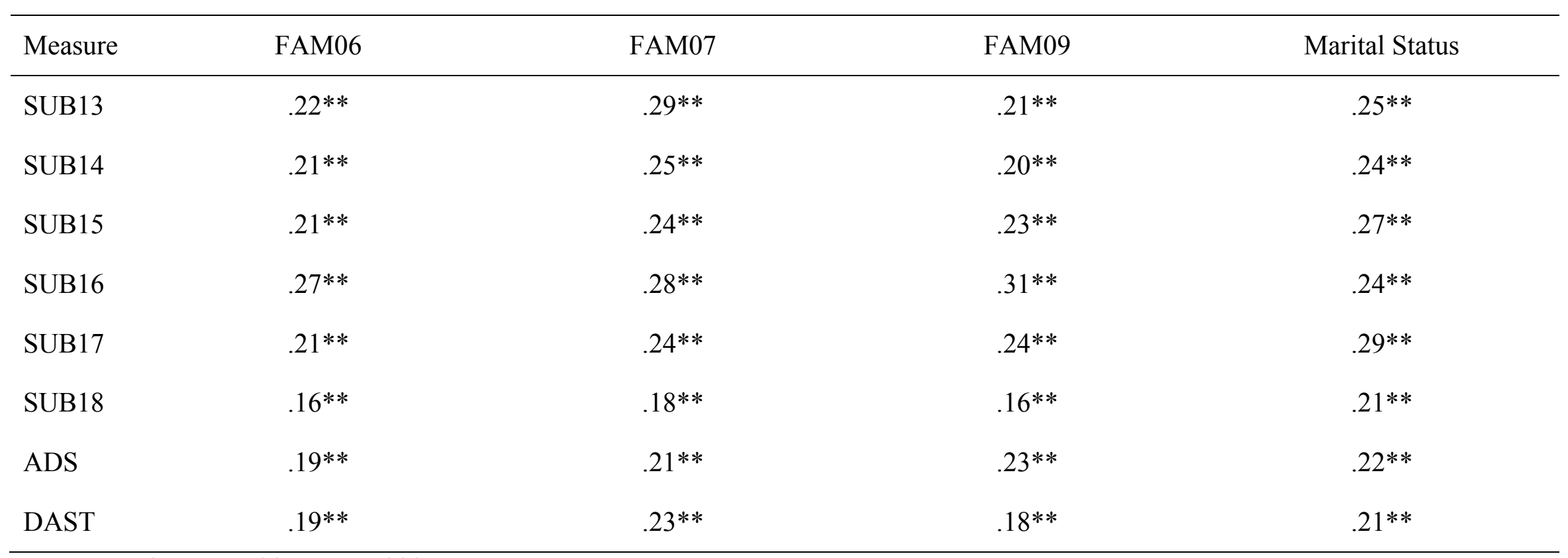

Note: ${ }^{*} p<.05 .{ }^{* *} p<.01 .{ }^{* * *} p<.001$ 


\section{Table C5}

Pearson Product Moment Correlations of the Gender-informed Substance Abuse Domain with Personal/Emotional Domain Using $N=1528$

\begin{tabular}{|c|c|c|c|c|c|c|c|}
\hline Measure & PER05 & PER06 & PER13 & PER15 & PER18 & PER19 & PER21 \\
\hline SUB01 & $.17 * *$ & $.18 * *$ & $.09 * *$ & $.10 * *$ & $.20 * *$ & $.23 * *$ & $.26 * *$ \\
\hline SUB03 & $.16^{* *}$ & $.19 * *$ & $.11 * *$ & $.18^{* *}$ & $.21 * *$ & $.24 * *$ & $.29 * *$ \\
\hline SUB05 & $.18^{* *}$ & $.20 * *$ & $.13 * *$ & $.20 * *$ & $.27 * *$ & $.23^{* *}$ & $.27 * *$ \\
\hline SUB06 & $.19 * *$ & $.17^{* *}$ & $.10^{* *}$ & $.18 * *$ & $.26 * *$ & $.23^{* *}$ & $.26^{* *}$ \\
\hline SUB07 & $.16^{* *}$ & $.15^{* *}$ & $.11 * *$ & $.19 * *$ & $.27 * *$ & $.24 * *$ & $.27 * *$ \\
\hline SUB11 & $.19 * *$ & $.20 * *$ & .05 & $.14 * *$ & $.13^{* *}$ & $.15^{* *}$ & $.20 * *$ \\
\hline SUB12 & $.21 * *$ & $.21 * *$ & $.05^{*}$ & $.14 * *$ & $.16^{* *}$ & $.18^{* *}$ & $\begin{array}{l}.26^{* *} \\
\text { (Cont }\end{array}$ \\
\hline
\end{tabular}




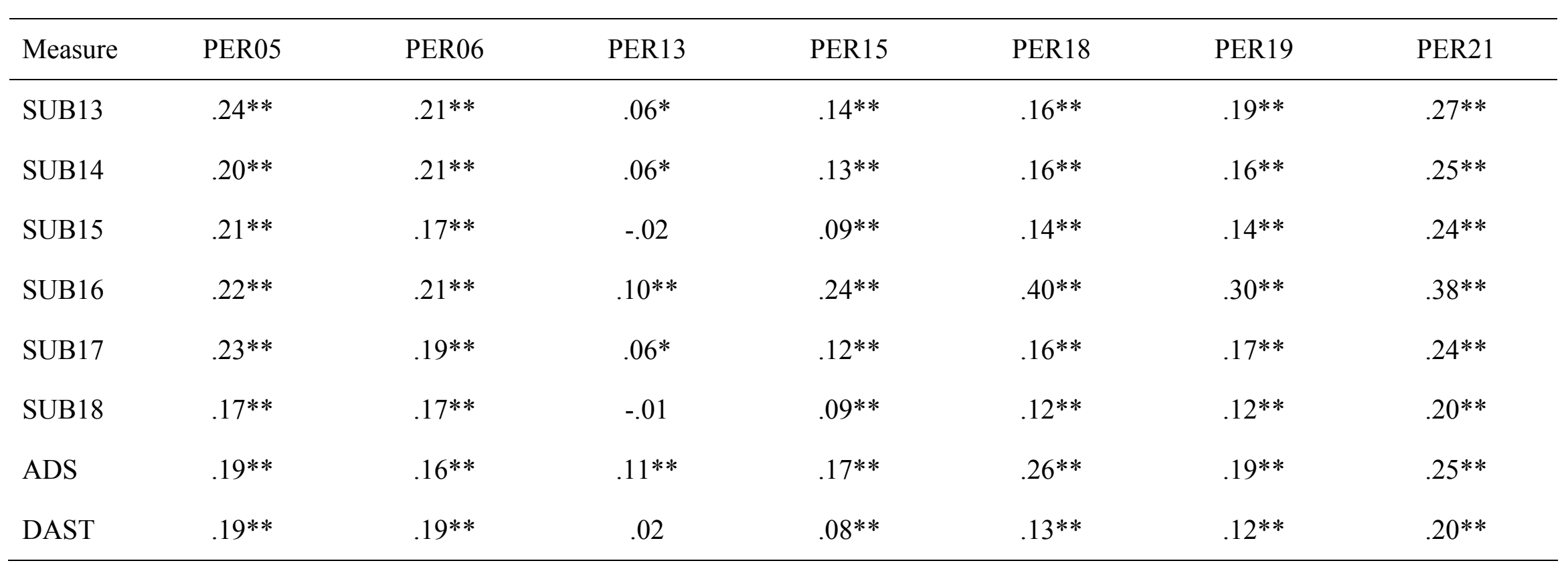

Note: ${ }^{*} p<.05 .{ }^{* *} p<.01 .{ }^{* * *} p<.001$ 


\section{Appendix D}

Correlation Matrix of Gender-informed Mental Health Domain

Table D1

Correlation Matrix of Gender-informed Mental Health Domain with Mental Health Domain Using N=1528

\begin{tabular}{|c|c|c|c|c|c|c|}
\hline Measure & 1 & 2 & 3 & 4 & 5 & 6 \\
\hline $\begin{array}{l}\text { 1. GSI subscale of the } \\
\text { Brief Symptom Inventory }\end{array}$ & - & & & & & \\
\hline 2. DHS Depression & $.79 * *$ & - & & & & \\
\hline $\begin{array}{l}\text { 3. DHS Current Suicidal } \\
\text { Ideation }\end{array}$ & $.37 * *$ & $.33 * *$ & - & & & \\
\hline 4. DHS Hopelessness & $.67 * *$ & $.73 * *$ & $.47 * *$ & - & & \\
\hline $\begin{array}{l}\text { 5. DHS Cognitive } \\
\text { Indicator }\end{array}$ & $.25^{* *}$ & $.21 * *$ & $.53 * *$ & $.32 * *$ & - & \\
\hline 6. DHS Historical Suicide & $.40 * *$ & $.39 * *$ & $.33 * *$ & $.37 * *$ & $.30 * *$ & - \\
\hline
\end{tabular}

Note: ${ }^{*} p<.05 .{ }^{* *} p<.01 .{ }^{* * *} p<.001$ 


\section{Appendix E}

Pearson Product Moment Correlations of the Gender-informed Mental Health Domain

Table E1.

Pearson Product Moment Correlations of the Gender-informed Mental Health Domain with Relationship Dysfunction Domain Using $N=1528$

\begin{tabular}{|c|c|c|c|c|}
\hline Measure & FAM06 & FAM07 & FAM09 & Marital Status \\
\hline $\begin{array}{l}\text { GSI subscale of the } \\
\text { Brief Symptom } \\
\text { Inventory }\end{array}$ & $.06^{*}$ & $.18^{* *}$ & .01 & -.03 \\
\hline DHS Depression & $.10 * *$ & $.18 * *$ & .03 & -.05 \\
\hline $\begin{array}{l}\text { DHS Current Suicidal } \\
\text { Ideation }\end{array}$ & .03 & .03 & .05 & -.04 \\
\hline DHS Hopelessness & $.10 * *$ & $.12 * *$ & .03 & -.06 \\
\hline $\begin{array}{l}\text { DHS Cognitive } \\
\text { Indicator }\end{array}$ & .03 & .05 & .04 & .02 \\
\hline $\begin{array}{l}\text { DHS Historical } \\
\text { Suicide }\end{array}$ & $.09 * *$ & $.15^{* *}$ & $.16^{* *}$ & -.02 \\
\hline
\end{tabular}


Table E2

Pearson Product Moment Correlations of the Gender-informed Mental Health Domain with Parental/Family Issues Domain Using $N=1528$

\begin{tabular}{|c|c|c|c|c|c|}
\hline Measure & FAM02 & FAM05 & FAM12 & ASSO09 & ASSO10 \\
\hline $\begin{array}{l}\text { GSI subscale of } \\
\text { the Brief Symptom } \\
\text { Inventory }\end{array}$ & $.19 * *$ & $.11 * *$ & $.10 * *$ & $.10^{* *}$ & $.18^{* *}$ \\
\hline DHS Depression & $.19 * *$ & $.11 * *$ & $.14 * *$ & $.11 * *$ & $.24 * *$ \\
\hline $\begin{array}{l}\text { DHS Current } \\
\text { Suicidal Ideation }\end{array}$ & $.09 * *$ & $.11^{* *}$ & -.01 & $.10^{* *}$ & $.09 * *$ \\
\hline DHS Hopelessness & $.10 * *$ & .04 & $.12 * *$ & $.11 * *$ & $.18 * *$ \\
\hline $\begin{array}{l}\text { DHS Cognitive } \\
\text { Indicator }\end{array}$ & .04 & .06 & -.04 & $.08^{*}$ & .05 \\
\hline $\begin{array}{l}\text { DHS Historical } \\
\text { Suicide }\end{array}$ & $.19^{* *}$ & $.16^{* *}$ & $.08^{*}$ & $.13^{* *}$ & $.17 * *$ \\
\hline
\end{tabular}


Table E3

Pearson Product Moment Correlations of the Gender-informed Mental Health Domain with Victimization Domain Using $N=1528$

\begin{tabular}{|c|c|c|c|c|}
\hline Measure & FAM01 & FAM03 & FAM04 & FAM08 \\
\hline $\begin{array}{l}\text { GSI subscale of the } \\
\text { Brief Symptom } \\
\text { Inventory }\end{array}$ & $.14^{* *}$ & $.16^{* *}$ & $.15^{* *}$ & $.15^{* *}$ \\
\hline DHS Depression & $.15 * *$ & $.18 * *$ & $.16^{* *}$ & $.17 * *$ \\
\hline $\begin{array}{l}\text { DHS Current Suicidal } \\
\text { Ideation }\end{array}$ & .06 & $.07 *$ & $.16^{* *}$ & $.17 * *$ \\
\hline DHS Hopelessness & $.09 * *$ & $.09 * *$ & $.10 * *$ & $.13 * *$ \\
\hline $\begin{array}{l}\text { DHS Cognitive } \\
\text { Indicator }\end{array}$ & .05 & $.06^{*}$ & .06 & .05 \\
\hline $\begin{array}{l}\text { DHS Historical } \\
\text { Suicide }\end{array}$ & $.19 * *$ & $.24 * *$ & $.18^{* *}$ & $.14^{* *}$ \\
\hline
\end{tabular}


Table E4

Pearson Product Moment Correlations of the Gender-informed Mental Health Domain with Personal/Emotional Domain Using $N=1528$

\begin{tabular}{|c|c|c|c|c|c|c|c|}
\hline Measure & PER05 & PER06 & PER13 & PER15 & PER18 & PER19 & PER21 \\
\hline $\begin{array}{l}\text { GSI subscale of } \\
\text { the Brief } \\
\text { Symptom } \\
\text { Inventory }\end{array}$ & $.19 * *$ & $.18^{* *}$ & $.11 * *$ & $.09 * *$ & $.09 * *$ & .03 & $.15 * *$ \\
\hline DHS Depression & $.21 * *$ & $.22 * *$ & $.12 * *$ & $.10^{* *}$ & $.09 * *$ & .04 & $.14 * *$ \\
\hline $\begin{array}{l}\text { DHS Current } \\
\text { Suicidal Ideation }\end{array}$ & $.12 * *$ & $.12 * *$ & $.07 *$ & $.08^{*}$ & $.17 * *$ & $.08 *$ & $.11 * *$ \\
\hline $\begin{array}{l}\text { DHS } \\
\text { Hopelessness }\end{array}$ & $.16^{* *}$ & $.18 * *$ & $.14^{* *}$ & $.09 * *$ & $.11 * *$ & .05 & $.13 * *$ \\
\hline $\begin{array}{l}\text { DHS Cognitive } \\
\text { Indicator }\end{array}$ & $.09 * *$ & $.09 * *$ & $.07 *$ & .05 & $.11 * *$ & $.07 *$ & $.11^{* *}$ \\
\hline $\begin{array}{l}\text { DHS Historical } \\
\text { Suicide }\end{array}$ & $.21 * *$ & $.17 * *$ & $.17 * *$ & $.18^{* *}$ & $.24 * *$ & $.16^{* *}$ & $.23 * *$ \\
\hline
\end{tabular}

Note: $* p<.05 . * * p<.01 . * * * p<.001$ 
Appendix F

Correlation Matrix of the Gender-informed Relationship Dysfunction Domain

Table F1

Correlation Matrix of Gender-informed Relationship Dysfunction Domain with Relationship Dysfunction Domain Using N=1528

\begin{tabular}{lcccc}
\hline \multicolumn{1}{c}{ Measure } & 1 & 2 & 3 & 4 \\
\hline 1. FAM06 & - & & \\
2. FAM07 & $.24^{* *}$ & - & - \\
3. FAM09 & $.16^{* *}$ & $.25^{* *}$ & .02 & - \\
4. Marital Status & $-.11^{* *}$ & -.04 &
\end{tabular}




\section{Appendix G}

Pearson Product Moment Correlations of the Gender-informed Relationship Dysfunction Domain

Table G1

Pearson Product Moment Correlations of the Gender-informed Relationship Dysfunction Domain with Parental/Family Issues Domain Using $N=1528$

\begin{tabular}{|c|c|c|c|c|c|}
\hline Measure & FAM02 & FAM05 & FAM12 & ASSO09 & ASSO10 \\
\hline FAM06 & $.23 * *$ & $.18^{* *}$ & $.16^{* *}$ & $.20 * *$ & $.22 * *$ \\
\hline FAM07 & $.23 * *$ & $.14^{* *}$ & $.23 * *$ & $.16^{* *}$ & $.23 * *$ \\
\hline FAM09 & $.19 * *$ & $.16^{* *}$ & $.21^{* *}$ & $.20 * *$ & $.21^{* *}$ \\
\hline Marital Status & -.04 & -.02 & .01 & -.04 & -.01 \\
\hline
\end{tabular}

Note: $* p<.05 . * * p<.01 . * * * p<.001$ 
Table G2

Pearson Product Moment Correlations of the Gender-informed Relationship Dysfunction Domain with Victimization Domain Using $N=1528$

\begin{tabular}{|c|c|c|c|c|}
\hline Measure & FAM01 & FAM03 & FAM04 & FAM08 \\
\hline FAM06 & $.22 * *$ & $.18^{* *}$ & $.19 * *$ & $.14^{* *}$ \\
\hline FAM07 & $.17 * *$ & $.21 * *$ & $.20 * *$ & $.54 * *$ \\
\hline FAM09 & $.16^{* *}$ & $.18 * *$ & $.21 * *$ & $.34 * *$ \\
\hline Marital Status & .00 & -.02 & -.01 & .02 \\
\hline
\end{tabular}

Table G3

Pearson Product Moment Correlations of the Gender-informed Relationship Dysfunction Domain with Personal/Emotional Domain Using $N=1528$

\begin{tabular}{lccccccc}
\hline \multicolumn{1}{c}{ Measure } & PER05 & PER06 & PER13 & PER15 & PER18 & PER19 & PER21 \\
\hline FAM06 & $.15^{* *}$ & $.28^{* *}$ & $.10^{* *}$ & $.22^{* *}$ & $.20^{* *}$ & $.14^{* *}$ & $.21^{* *}$ \\
FAM07 & $.22^{* *}$ & $.17^{* *}$ & $.14^{* *}$ & $.28^{* *}$ & $.13^{* *}$ & $.18^{* *}$ & $.18^{* *}$ \\
FAM09 & $.14^{* *}$ & $.12^{* *}$ & $.07^{* *}$ & $.25^{* *}$ & $.30^{* *}$ & $.23^{* *}$ & $.35^{* *}$ \\
Marital Status & .02 & -.03 & .00 & -.04 & $-.06^{*}$ & -.01 & -.01 \\
\hline Note: $* p<.05 . * * p<.01 . * * * p<.001$ & & & &
\end{tabular}


Appendix H

Correlation Matrix of the Gender-informed Parental/Family Issues Domain

Table H1

Correlation Matrix of Gender-informed Parental/Family Issues Domain with Parental/Family Issues Domain Using N=1528

\begin{tabular}{|c|c|c|c|c|c|}
\hline Measure & 1 & 2 & 3 & 4 & 5 \\
\hline 1. FAM02 & - & & & & \\
\hline 2. FAM05 & $.40 * *$ & - & & & \\
\hline 3. FAM12 & $.24 * *$ & $.15^{* *}$ & - & & \\
\hline 4. ASSO09 & $.39 * *$ & $.28 * *$ & $.24 * *$ & - & \\
\hline 5. ASSO10 & $.29 * *$ & $.25^{* *}$ & $.30 * *$ & $.42 * *$ & - \\
\hline
\end{tabular}

Note: ${ }^{*} p<.05 . * * p<.01 .{ }^{* * *} p<.001$ 


\section{Appendix I}

Pearson Product Moment Correlations for the Gender-informed Parental/Family Issues Domain

Table I1.

Pearson Product Moment Correlations of the Gender-informed Parental/Family Issues Domain with Victimization Domain Using $N=1528$

\begin{tabular}{lcccc}
\hline Measure & FAM01 & FAM03 & FAM04 & FAM08 \\
\hline FAM02 & $.59^{* *}$ & $.62^{* *}$ & $.58^{* *}$ & $.24 * *$ \\
FAM05 & $.36^{* *}$ & $.38^{* *}$ & $.43^{* *}$ & $.17^{* *}$ \\
FAM12 & $.20^{* *}$ & $.19^{* *}$ & $.19^{* *}$ & $.28^{* *}$ \\
ASSO09 & $.41^{* *}$ & $.32^{* *}$ & $.28^{* *}$ & $.16^{* *}$ \\
ASSO10 & $.27^{* *}$ & $.22^{* *}$ & $.22 * *$ & $.24 * *$ \\
\hline
\end{tabular}

Note: ${ }^{*} p<.05 .{ }^{* *} p<.01 .{ }^{* * *} p<.001$ 
Table I2

Pearson Product Moment Correlations of the Gender-informed Parental/Family Issues Domain with Personal/Emotional Domain Using $N=1528$

\begin{tabular}{|c|c|c|c|c|c|c|c|}
\hline Measure & PER05 & PER06 & PER13 & PER15 & PER18 & PER19 & PER21 \\
\hline FAM02 & $.22 * *$ & $.20 * *$ & $.14 * *$ & $.22 * *$ & $.22 * *$ & $.22 * *$ & $.26^{* *}$ \\
\hline FAM05 & $.16^{* *}$ & $.17 * *$ & $.09 * *$ & $.11 * *$ & $.18 * *$ & $.20 * *$ & $.22 * *$ \\
\hline FAM12 & $.22 * *$ & $.27 * *$ & $.09 * *$ & $.23 * *$ & $.16^{* *}$ & $.19 * *$ & $.22 * *$ \\
\hline ASSO09 & $.21 * *$ & $.24 * *$ & $.11 * *$ & $.24 * *$ & $.22 * *$ & $.19 * *$ & $.26^{* *}$ \\
\hline ASSO10 & $.24 * *$ & $.24 * *$ & $.11 * *$ & $.23 * *$ & $.21 * *$ & $.22 * *$ & $.28 * *$ \\
\hline
\end{tabular}

Note: $* p<.05 . * * p<.01 . * * * p<.001$ 


\section{Appendix J}

\section{Correlation Matrix of the Gender-informed Victimization Domain}

Table J1

Correlation Matrix of Gender-informed Victimization Domain with Victimization Domain Using N=1528

\begin{tabular}{lccc}
\hline \multicolumn{1}{c}{ Measure } & 1 & 2 & 3 \\
\hline 1. FAM01 & - & & \\
2. FAM03 & $.45^{* *}$ & - & - \\
3. FAM04 & $.40^{* *}$ & $.56^{* *}$ & $.23 * *$ \\
4. FAM08 & $.17^{* *}$ & $.27^{* *}$ & - \\
\hline
\end{tabular}

Note: ${ }^{*} p<.05 . * * p<.01 .{ }^{* * *} p<.001$ 


\section{Appendix K}

\section{Pearson Product Moment Correlations for the Gender-informed Victimization Domain}

Table K1

Pearson Product Moment Correlations of the Gender-informed Victimization Domain with Personal/Emotional Domain Using $N=1528$

\begin{tabular}{cccccccc}
\hline Measure & PER05 & PER06 & PER13 & PER15 & PER18 & PER19 & PER21 \\
\hline 1. FAM01 & $.16^{* *}$ & $.23^{* *}$ & $.11^{* *}$ & $.20^{* *}$ & $.22^{* *}$ & $.19^{* *}$ & $.23^{* *}$ \\
2. FAM03 & $.28^{* *}$ & $.21^{* *}$ & $.13^{* *}$ & $.20^{* *}$ & $.22^{* *}$ & $.24^{* *}$ & $.25^{* *}$ \\
3. FAM04 & $.20^{* *}$ & $.16^{* *}$ & $.12^{* *}$ & $.19^{* *}$ & $.21^{* *}$ & $.22^{* *}$ & $.20^{* *}$ \\
4. FAM08 & $.23^{* *}$ & $.18^{* *}$ & $.15^{* *}$ & $.20^{* *}$ & $.14^{* *}$ & $.18^{* *}$ & $.18^{* *}$ \\
\hline
\end{tabular}

Note: ${ }^{*} p<.05 .{ }^{* *} p<.01 .{ }^{* * *} p<.001$ 
Appendix L

Correlation Matrix for the Gender-informed Personal/Emotional Domain

Table L1

Correlation Matrix of Gender-informed Personal/Emotional Domain with Personal/Emotional Domain Using N=1528

\begin{tabular}{|c|c|c|c|c|c|c|c|}
\hline Measure & 1 & 2 & 3 & 4 & 5 & 6 & 7 \\
\hline 1. PER05 & - & & & & & & \\
\hline 2. PER06 & $.30 * *$ & - & & & & & \\
\hline 3. PER13 & $.19 * *$ & $.23 * *$ & - & & & & \\
\hline 4. PER15 & $.32 * *$ & $.26 * *$ & $.22 * *$ & - & & & \\
\hline 5. PER18 & $.19 * *$ & $.21 * *$ & $.13 * *$ & $.29 * *$ & - & & \\
\hline 6. PER19 & $.22 * *$ & $.16^{* *}$ & $.26 * *$ & $.29 * *$ & $.40 * *$ & - & \\
\hline 7. PER21 & $.24 * *$ & $.30 * *$ & $.15^{* *}$ & $.33 * *$ & $.51 * *$ & $.39 * *$ & - \\
\hline
\end{tabular}

Note: $* p<.05 . * * p<.01 . * * * p<.001$ 


\section{Appendix M}

ROC Curves for Any Institutional Misconduct Charges for the Total Sample

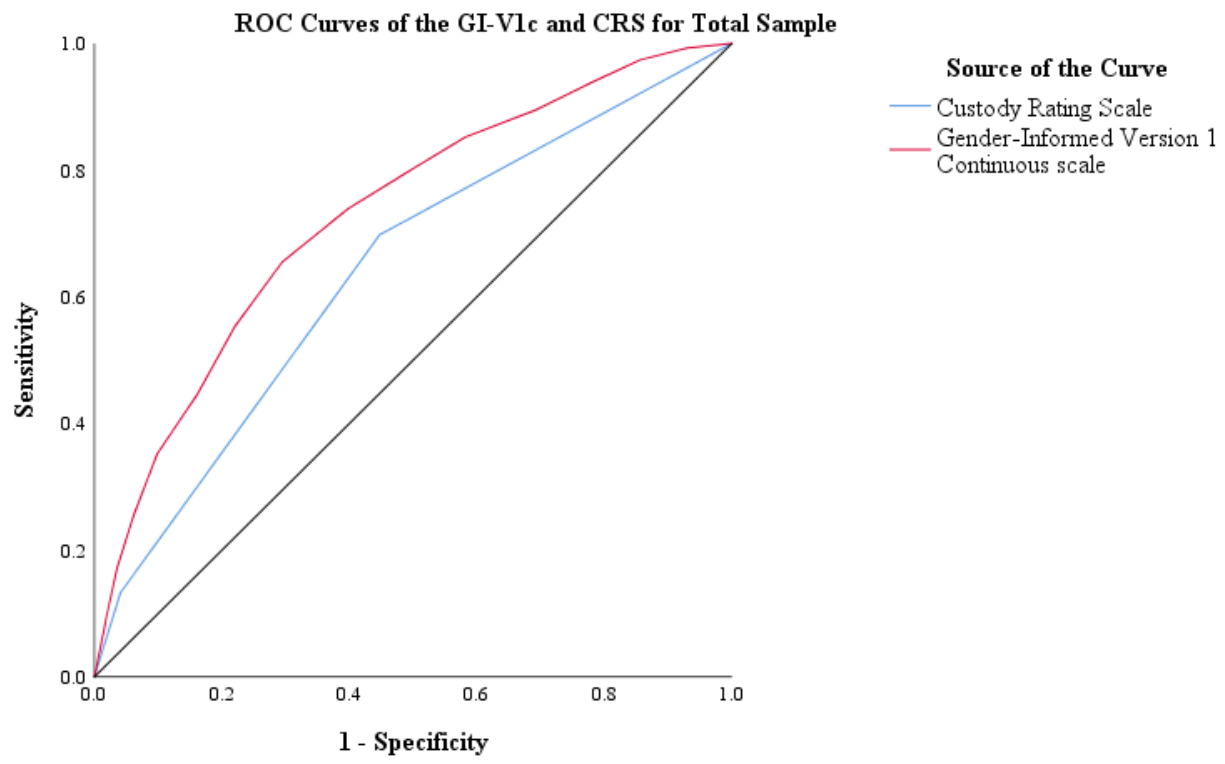

Figure 4. ROC Curves of the Gender-Informed Version 1 Continuous scale and Custody Rating Scale for any institutional misconduct charges.

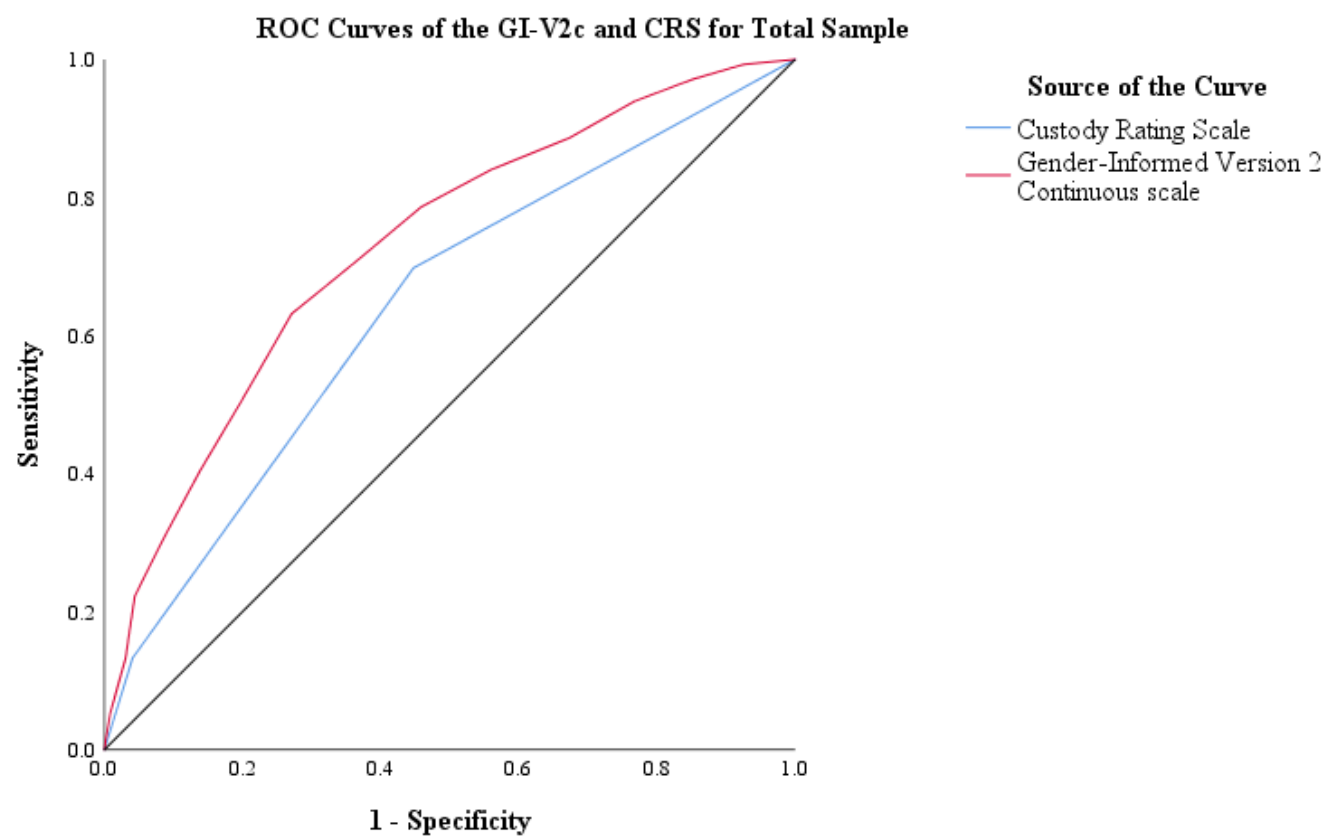

Figure 5. ROC Curves of the Gender-Informed Version 2 Continuous scale and Custody Rating Scale for any institutional misconduct charges. 


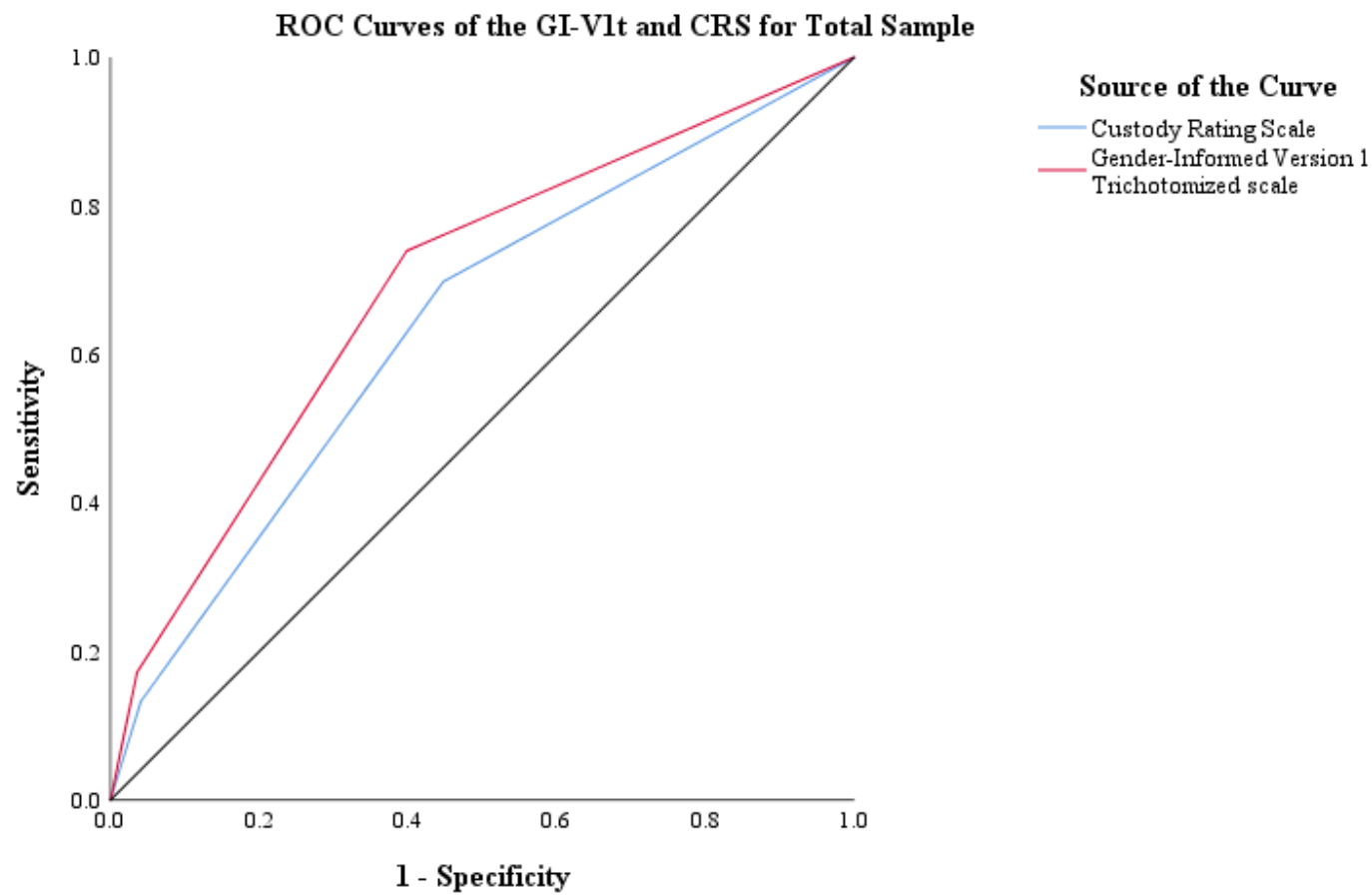

Figure 6. ROC Curves of the Gender-Informed Version 1 Trichotomized scale and Custody Rating Scale for any institutional misconduct charges.

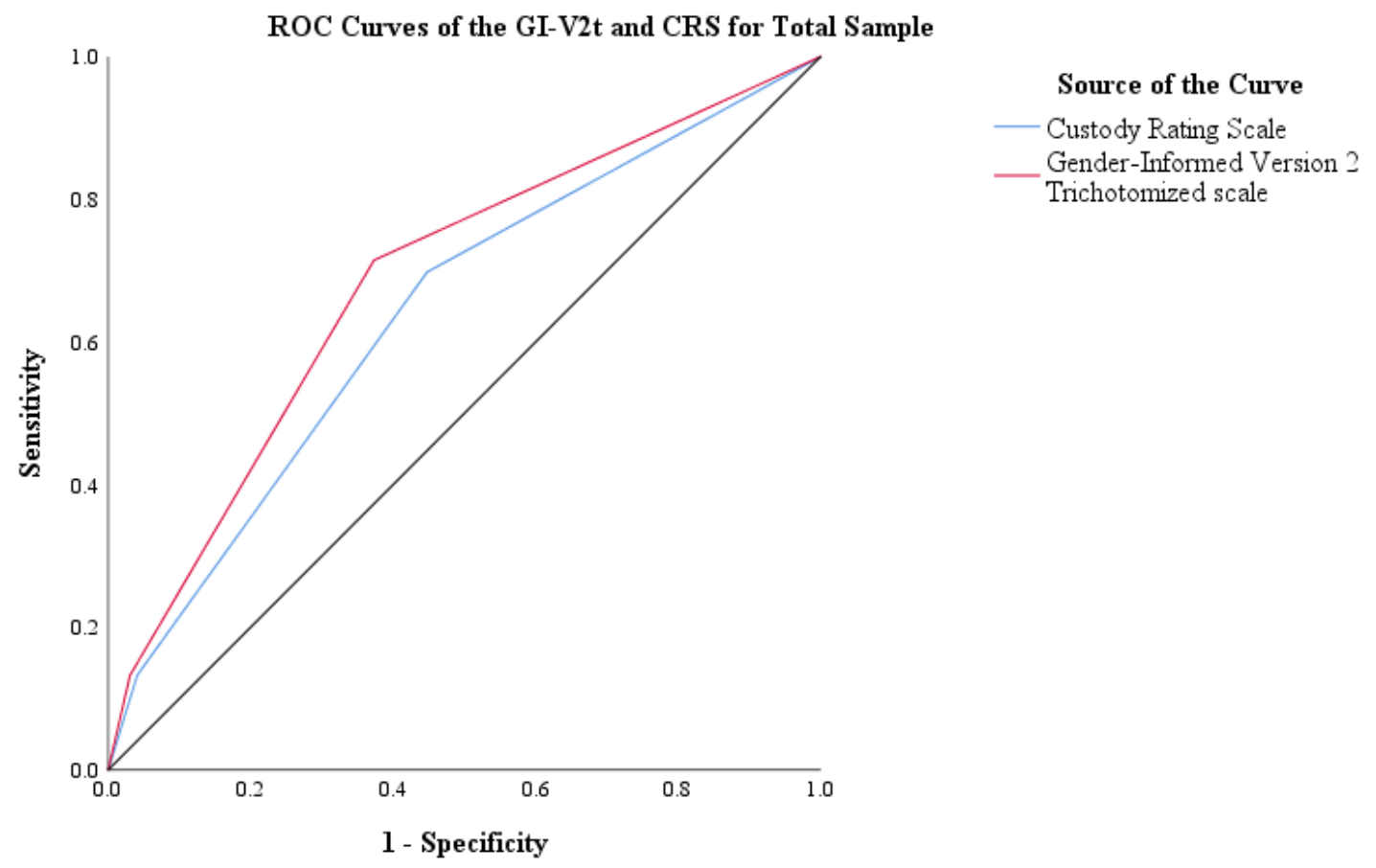

Figure 7. ROC Curves of the Gender-Informed Version 2 Trichotomized scale and Custody Rating Scale for any institutional misconduct charges. 


\section{Appendix N}

ROC Curves for Any Institutional Misconduct Charges by Indigenous Status

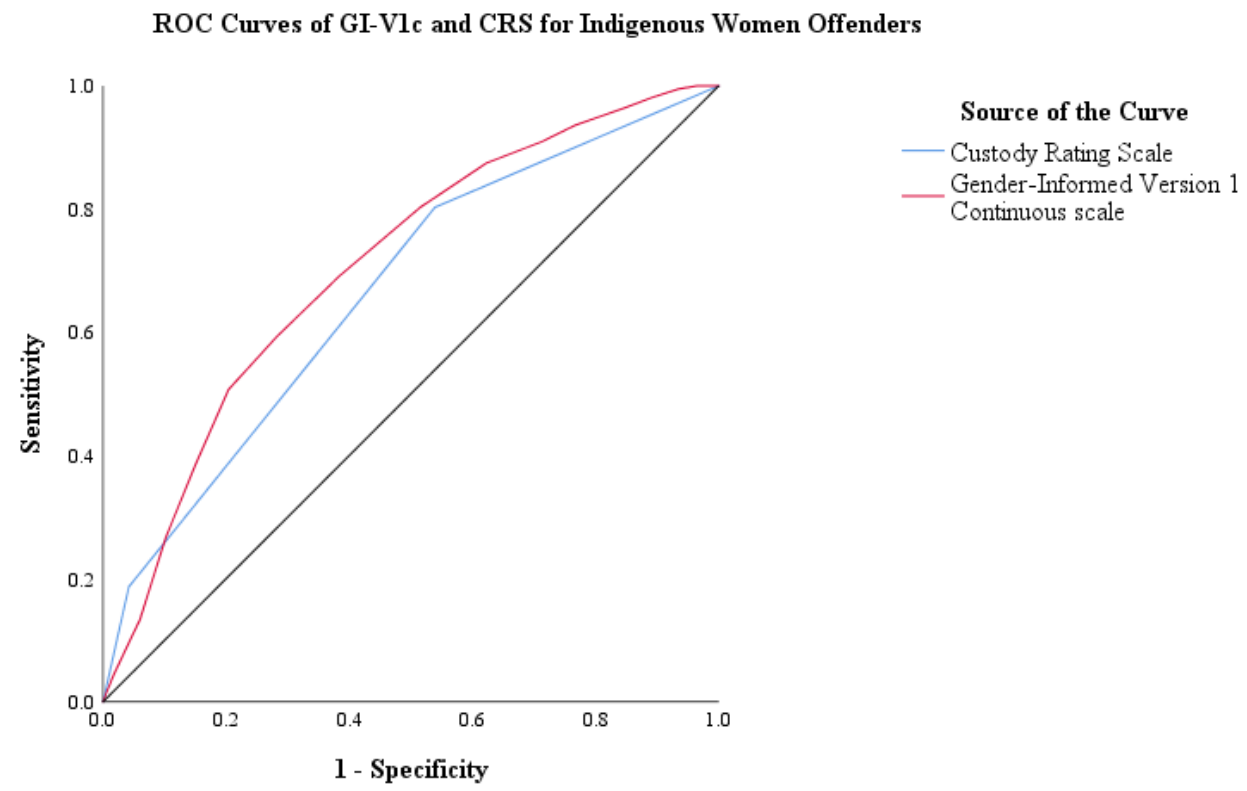

Figure 8. ROC Curves of the Gender-Informed Version 1 Continuous scale and Custody Rating Scale for any institutional misconduct charges for Indigenous women offenders.

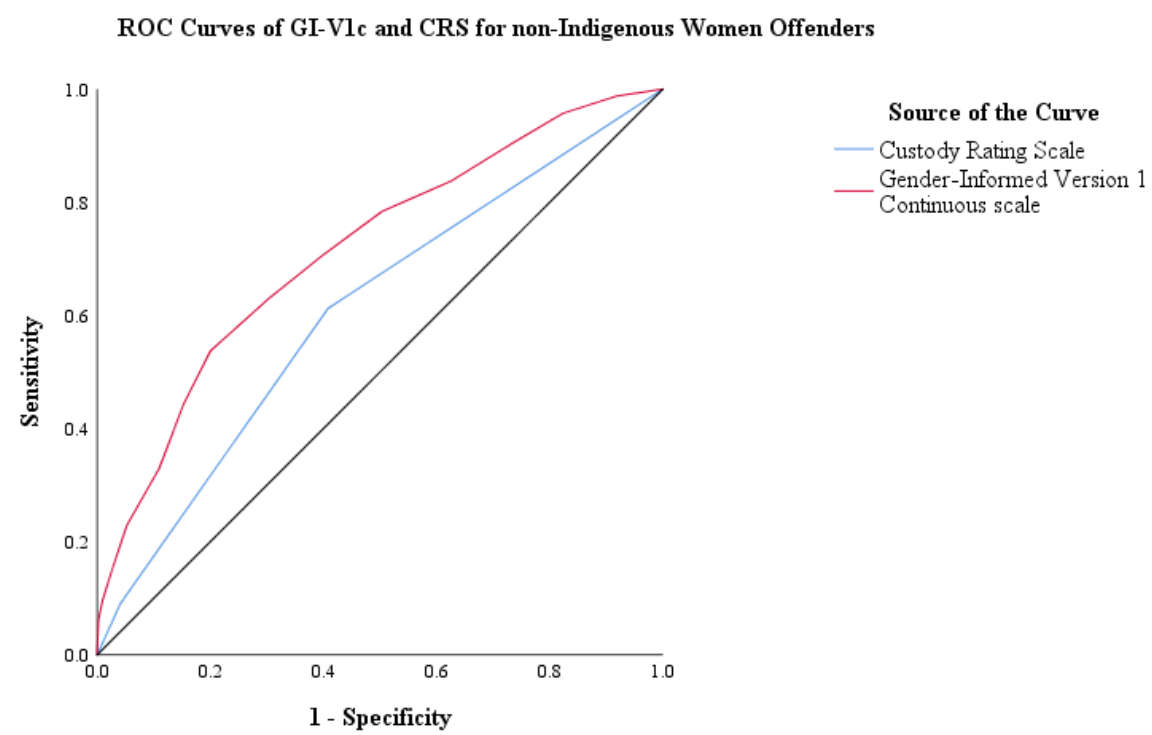

Figure 9. ROC Curves of the Gender-Informed Version 1 Continuous scale and Custody Rating Scale for any institutional misconduct charges for non-Indigenous women offenders. 


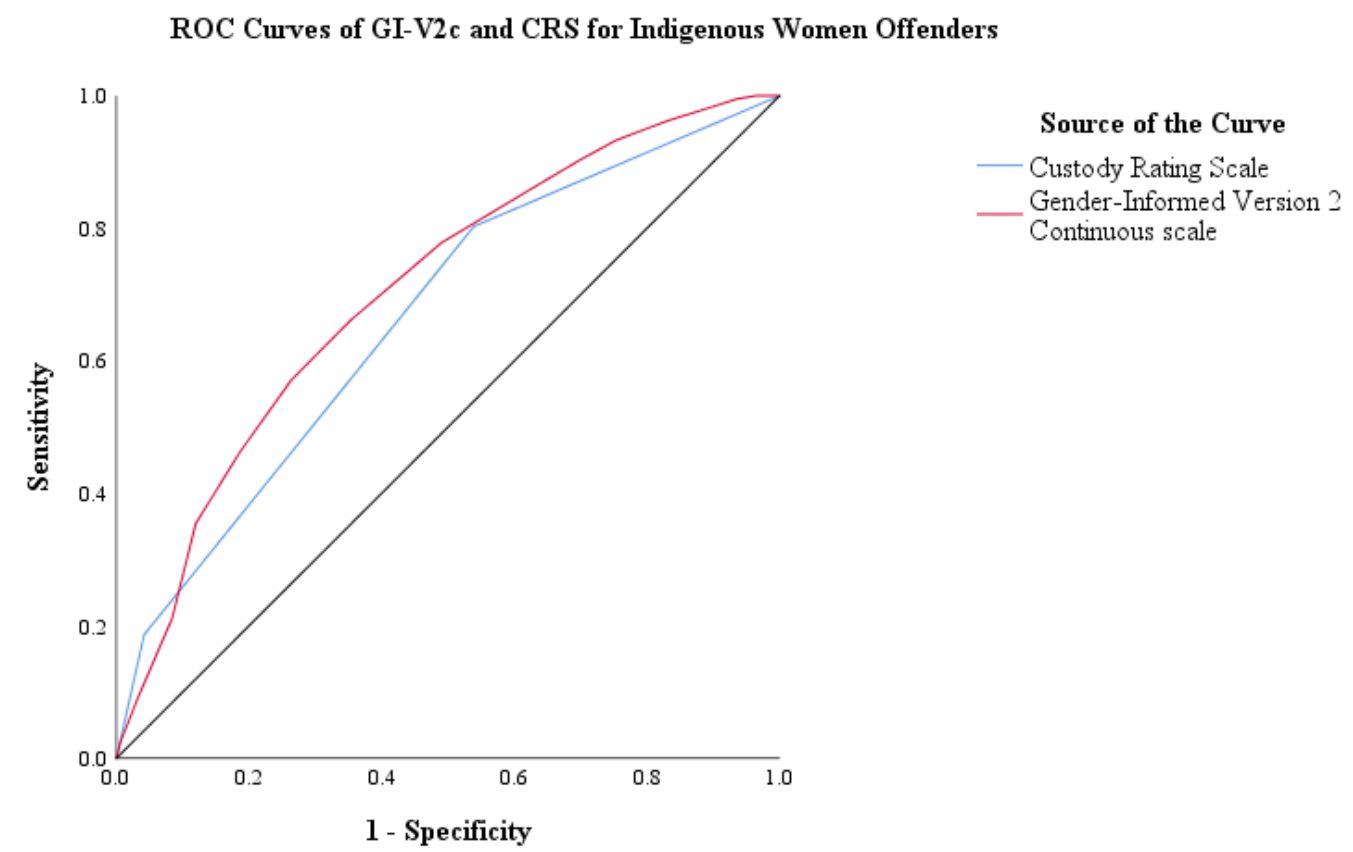

Figure 10. ROC Curves of the Gender-Informed Version 2 Continuous scale and Custody Rating Scale for any institutional misconduct charges for Indigenous women offenders.

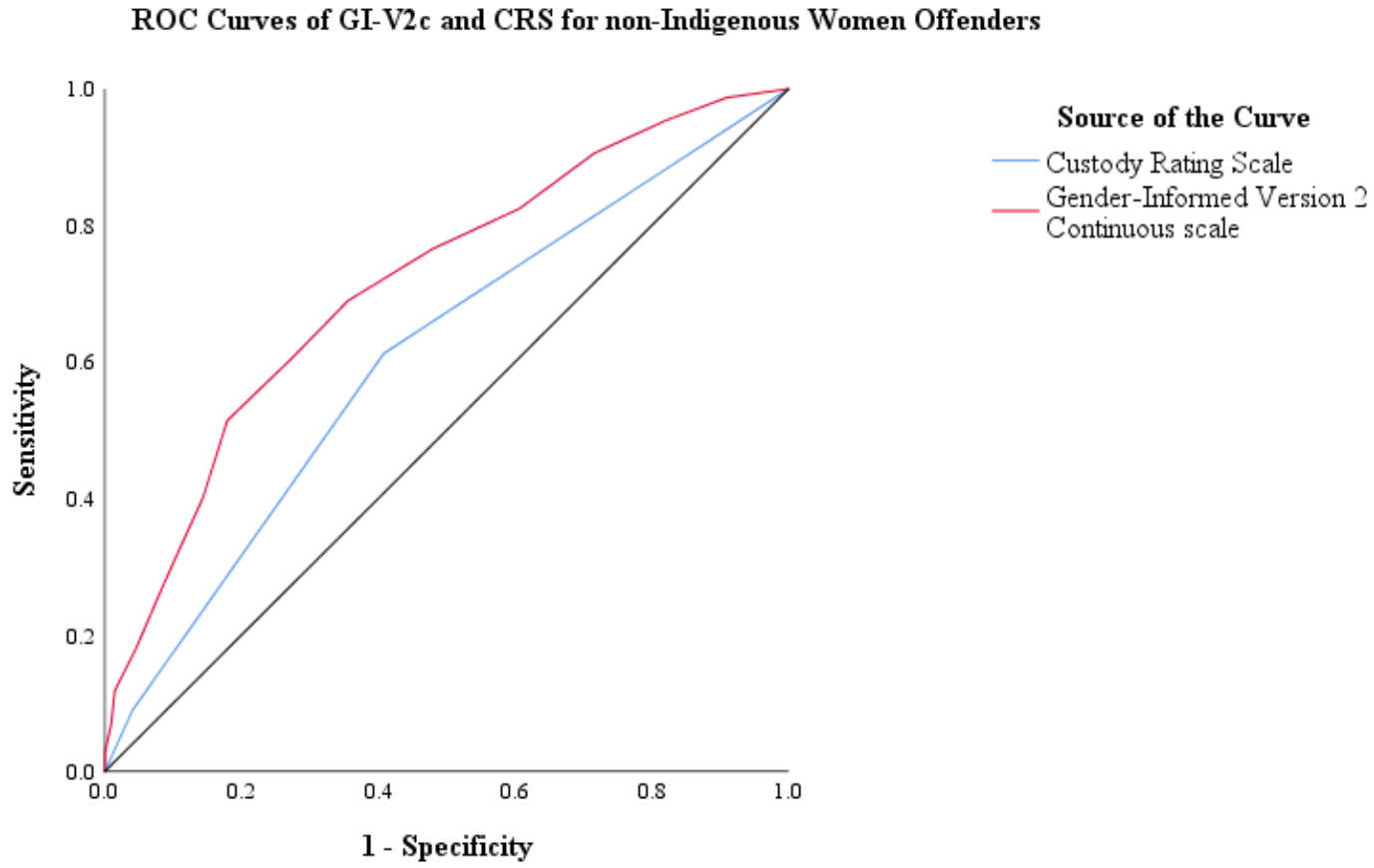

Figure 11. ROC Curves of the Gender-Informed Version 2 Continuous scale and Custody Rating Scale for any institutional misconduct charges for non-Indigenous women offenders. 


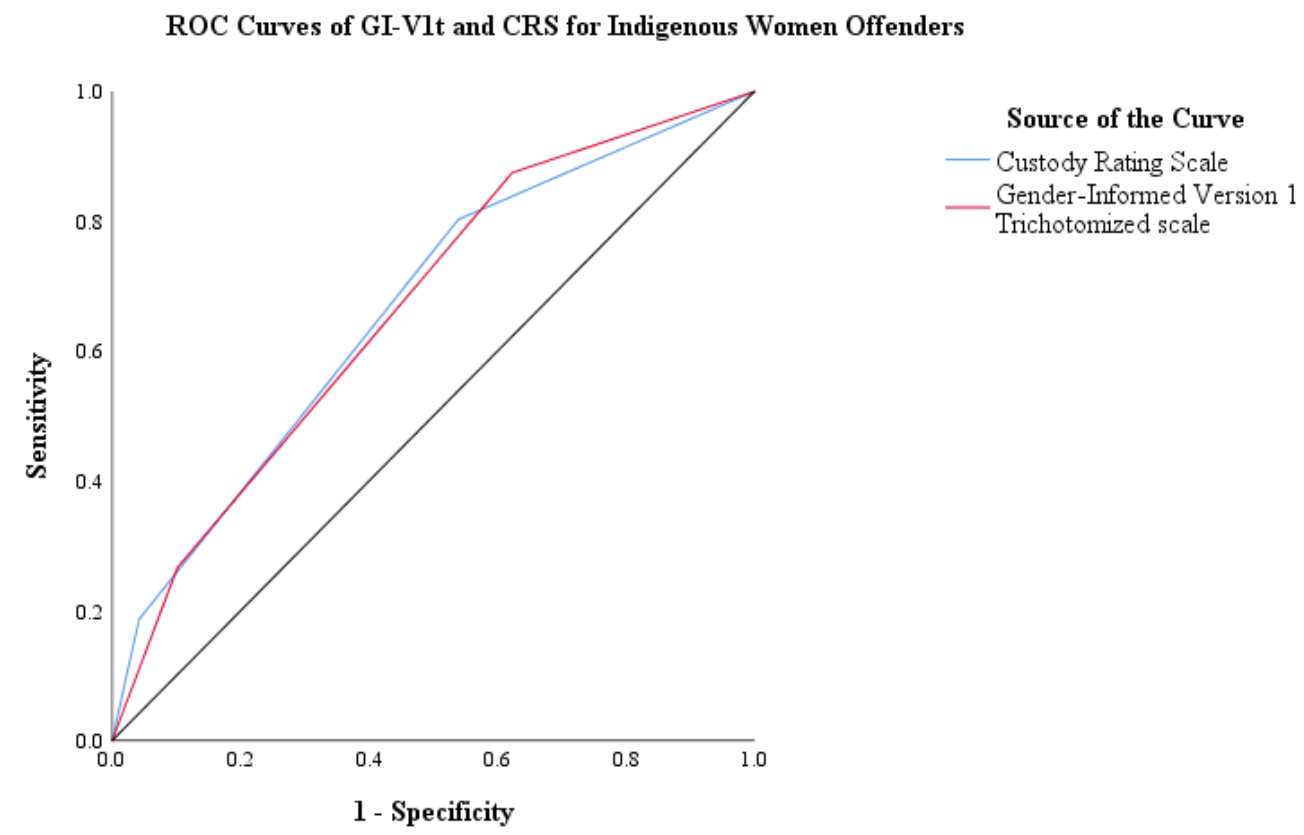

Figure 12. ROC Curves of the Gender-Informed Version 1 Trichotomized scale and Custody Rating Scale for any institutional misconduct charges for Indigenous women offenders.

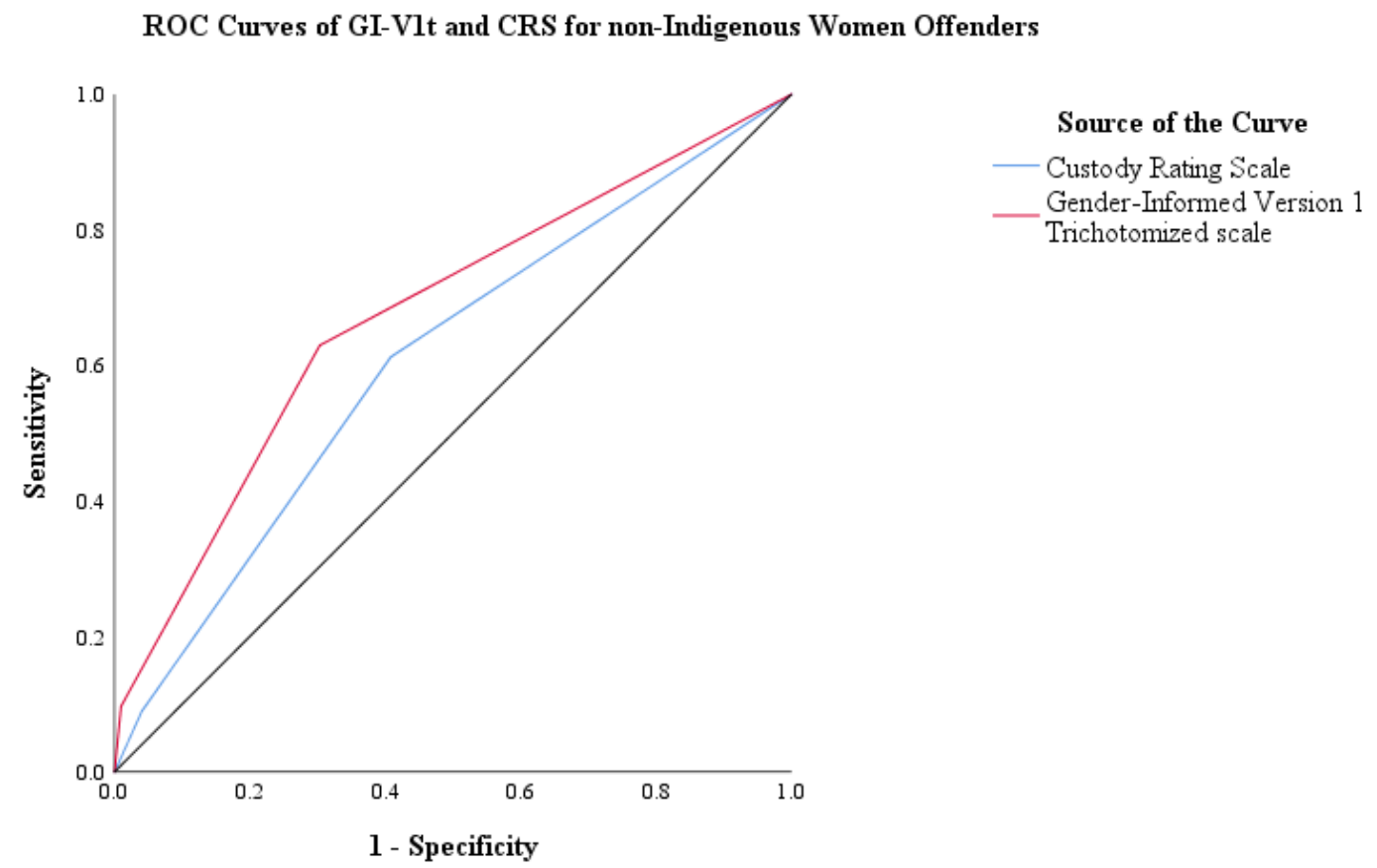

Figure 13. ROC Curves of the Gender-Informed Version 1 Trichotomized scale and Custody Rating Scale for any institutional misconduct charges for non-Indigenous women offenders. 


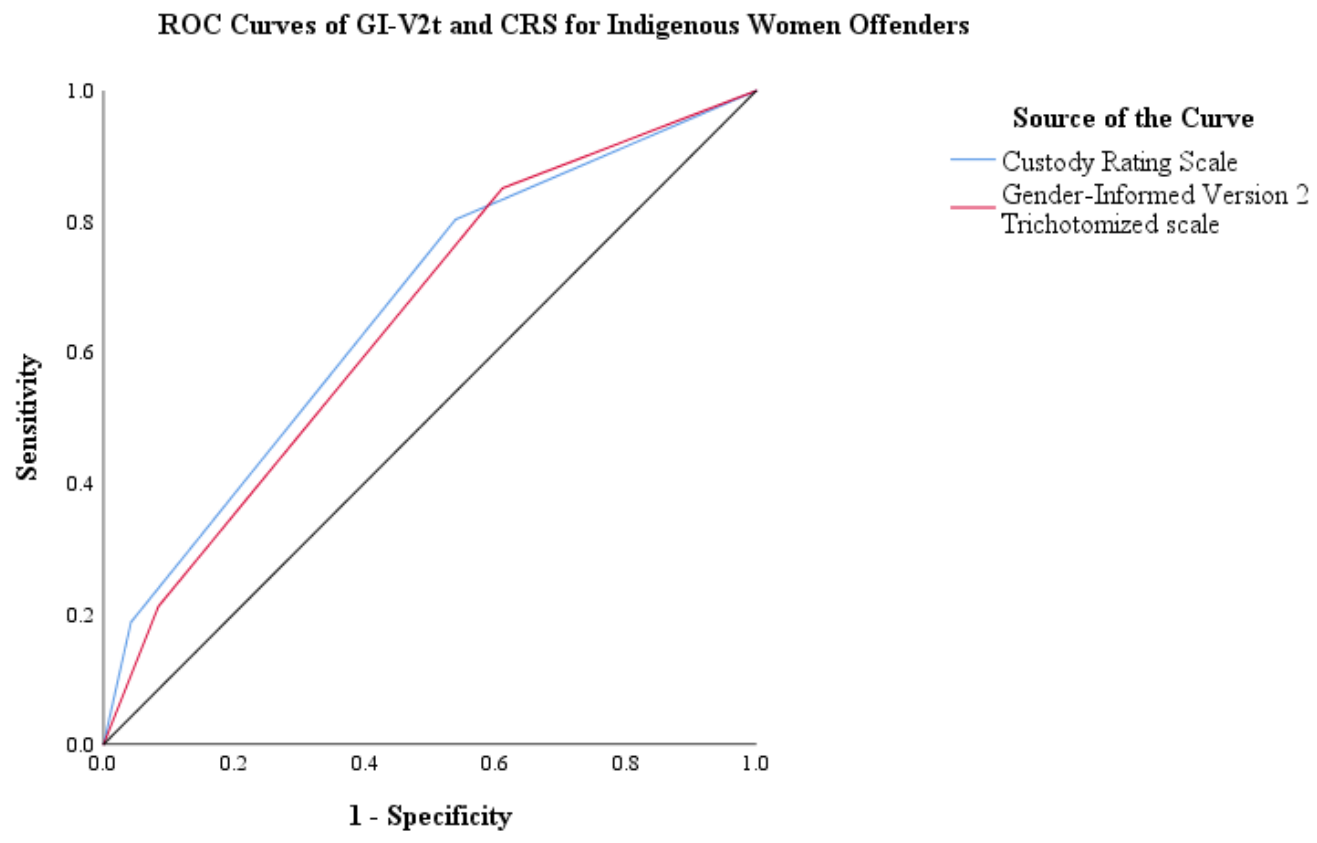

Figure 14. ROC Curves of the Gender-Informed Version 2 Trichotomized scale and Custody Rating Scale for any institutional misconduct charges for Indigenous women offenders.

ROC Curves of GI-V2t and CRS for non-Indigenous Women Offenders
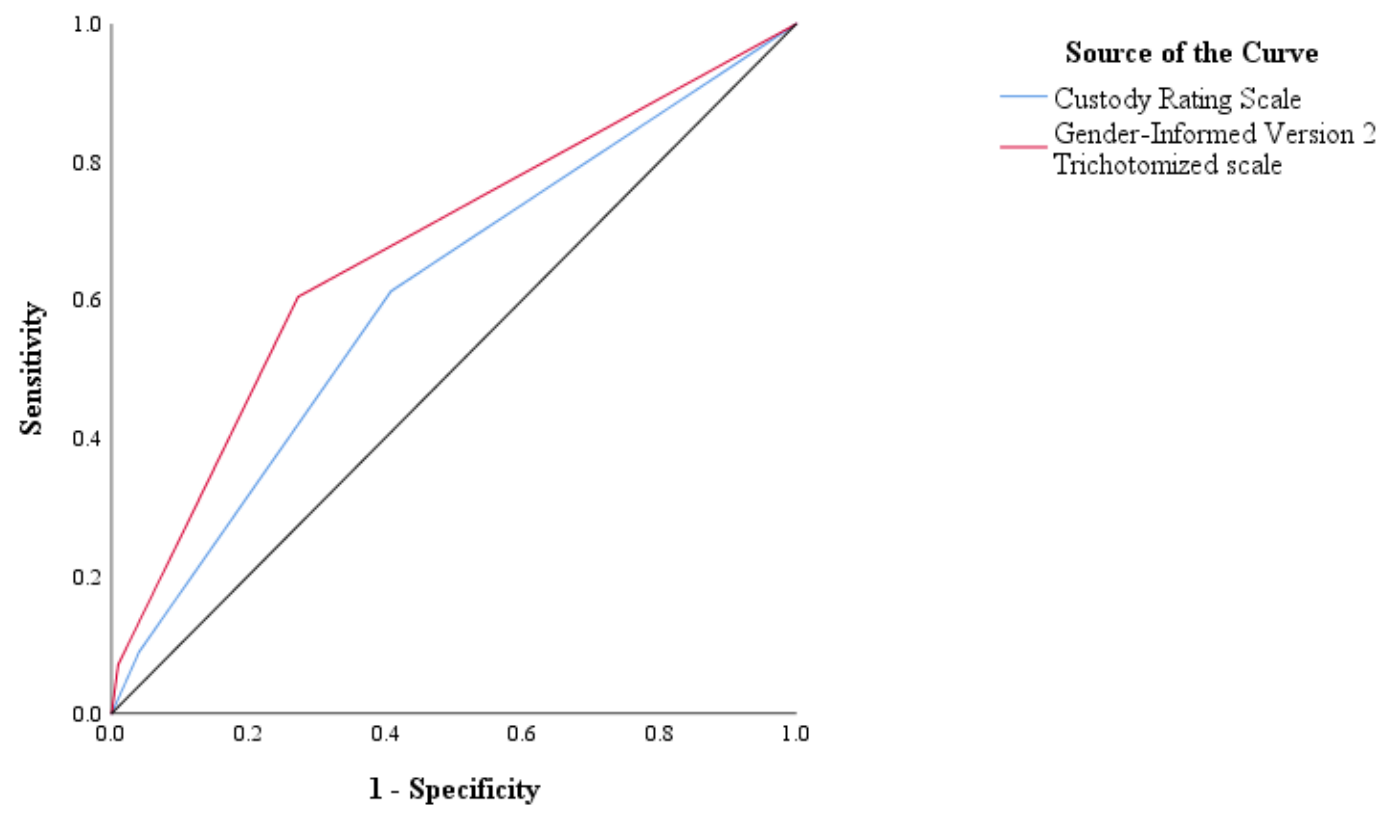

Figure 15. ROC Curves of the Gender-Informed Version 2 Trichotomized scale and Custody Rating Scale for any institutional misconduct charges for non-Indigenous women offenders. 